\title{
CAN A POPULATION SURVIVE IN A SHIFTING ENVIRONMENT USING NON-LOCAL DISPERSION
}

\author{
JÉRÔME COVILLE
}

\begin{abstract}
In this article, we analyse the non-local model :

$$
\partial_{t} U(t, x)=J \star U(t, x)-U(t, x)+f(x-c t, U(t, x)) \text { for } t>0 \text {, and } x \in \mathbb{R},
$$

where $J$ is a positive continuous dispersal kernel and $f(x, s)$ is a heterogeneous KPP type non-linearity describing the growth rate of the population. The ecological niche of the population is assumed to be bounded (i.e. outside a compact set, the environment is assumed to be lethal for the population) and shifted through time at a constant speed $c$. For compactly supported dispersal kernels $J$, assuming that for $c=0$ the population survive, we prove that there exists a critical speeds $c^{*, \pm}$ and $c^{* *, \pm}$ such that for all $-c^{*,-}<c<c^{*,+}$ then the population will survive and will perish when $c \geq c^{* *,+}$ or $c \leq-c^{* *,-}$. To derive this results we first obtain an optimal persistence criteria depending of the speed $c$ for non local problem with a drift term. Namely, we prove that for a positive speed $c$ the population persists if and only if the generalized principal eigenvalue $\lambda_{p}$ of the linear problem

$$
c \mathfrak{D}_{x}[\varphi]+J \star \varphi-\varphi+\partial_{s} f(x, 0) \varphi+\lambda_{p} \varphi=0 \quad \text { in } \quad \mathbb{R},
$$

is negative. $\lambda_{p}$ is a spectral quantity that we defined in the spirit of the generalized first eigenvalue of an elliptic operator. The speeds $c^{*, \pm}$ and $c^{* *, \pm}$ are then obtained through a fine analysis of the properties of $\lambda_{p}$ with respect to $c$. In particular, we establish its continuity with respect to the speed $c$. In addition, for any continuous bounded non-negative initial data, we establish the long time behaviour of the solution $U(t, x)$.
\end{abstract}

\section{INTRODUCTION}

Environmental changes due to earth global warming are enforcing species to shift their ranges to more favorable habitat region e.g. to the north or upward in elevation [39, 44, 46, 57]. The understanding of this transition and its consequence on the global diversity is of prime interest and numerous type of models have been considered to understand, evaluate and/or highlight this complex dynamical process and its impact [2, 6, 13, 17, 36, 38, 48, 58,

In this article, we are interested in the influence of long range dispersal processes for species living in a shifting environment caused by such environmental changes. For such a model species, we can think of trees of which seeds and pollens are disseminated on a wide range and whose environment changes through time as a consequence of a climate change. This possibility of a long range dispersal is well known in ecology, where numerous data now available support this assumptions [19, 20, 21, 51].

One way to model long range dispersal in the context of a shifting environment is to consider a population described by a shifted integrodifference model, that is, modelling the population by a density $n(t, x)$ whose time evolution (growth and dispersal) is governed by the following discrete in time equation :

$$
n_{t+1}(x)=\int_{-\frac{L}{2}+c t}^{\frac{L}{2}+c t} J(x, y) f\left[n_{t}(y)\right] d y,
$$

where $J$ is a dispersal kernel describing the movement of individuals between the time $t$ and $t+1, c>0$ is the speed of the environmental changes and $f$ is a growth function describing the demography of the species.

In this setting, Zhou and its collaborators show in [58, 59] how the speed of change $c$ affects the persistence of the population. Showing in particular that for too large speed of change $c$, the population will then go extinct whereas for small value of $c$, the species will be able to adapt and survive. They also suggest the existence of a critical speed $c^{*}>0$ for which for all speed $c<c^{*}$ then the population survive 
and goes extinct if $c \geq c^{*}$. For particular kernels and growth function, they provide some heuristics to compute this $c^{*}$.

Another commonly used model that integrates such long range dispersal is the following nonlocal reaction diffusion equation ([33, 35, 40, 43, 54]):

$$
\partial_{t} U(t, x)=J \star U(t, x)-U(t, x)+f(x-c t, U(t, x)) \text { for } t>0, \text { and } x \in \mathbb{R} .
$$

Here also $U(t, x)$ is the density of the considered population, $J$ is a dispersal kernel, $f(x, s)$ is a KPP type non-linearity describing the growth rate of the population and $c$ is the speed of the environmental changes. Compare to (1.1), the equation (1.2) is defined for all times $t$ and the movement and growth processes are not any more entangled.

In this particular setting the tail of the kernel can be thought of as the range of dispersion or as a measure of the frequency at which long dispersal events occur, reflecting at the population level some intrinsic variability in the capacity of each individual to disperse [37, 47].

The aim of this article is to investigate, as for integrodifference models, how the speed of the environmental change affects the survival of the population modelled by (1.2). Throughout this paper we will always make the following assumptions on the dispersal kernel $J$.

$$
J \in C(\mathbb{R}) \cap L^{1}(\mathbb{R}) \text { is nonnegative and of unit mass (i.e. } \int_{\mathbb{R}} J(z) d z=1 \text { ). }
$$

$$
J(0)>0
$$

There are many ways to model the impact of a changing environment. For example, Li, Wang and Zhao [42] consider an habitat which gradually change from a bad environment to a good environment. Namely, they consider $f(x-c t, s):=s(r(x-c t)-s)$ with $r(z)$ a continuous non decreasing function such that $\lim _{z \rightarrow-\infty} r(z)<0<\lim _{z \rightarrow+\infty} r(z)$. For such shrinking environment and for thin tailed kernel (i.e. $J$ such that $\left.\int_{\mathbb{R}} J(z) e^{\lambda z} d z<+\infty\right)$ the authors prove the existence of a critical speed $c^{*}$ defining the threshold between persistence and extinction. That is for a speed of change $c \geq c^{*}$ then the population will not survive whereas a monotone front will exist when $c<c^{*}$. Moreover the critical speed is defined by the following spectral formula :

$$
c^{*}:=\inf _{\lambda>0} \frac{1}{\lambda}\left(\int_{\mathbb{R}} J(z) e^{\lambda z}-1+\sup _{x \in \mathbb{R}} r(x)\right) .
$$

Here, instead of a monotone habitat whose favourable part shrinks through time, we focus our analysis on species that have a bounded ecological niche that shift with speed $c$. A simple way to model such a spatial repartition consists in considering that the environment is hostile to the species outside a bounded set. For instance, biological populations that are sensitive to temperature thrive only in a limited latitude zone. Thus, if $x$ is the latitude, we get such dependence. This fact is translated in our model by assuming that $f$ satisfies:

$$
f \in C(\mathbb{R} \times[0,+\infty)) \text { is smooth and of KPP type }
$$

that is

$f$ is differentiable with respect to $s$,

$\forall s \geq 0, f(\cdot, s) \in C^{0,1}(\mathbb{R})$,

$f(\cdot, 0) \equiv 0$,

For all $x \in \mathbb{R}, f(x, s) / s$ is decreasing with respect to $s$ on $(0,+\infty)$.

There exists $S(x) \in C(\mathbb{R}) \cap L^{\infty}(\mathbb{R})$ such that $f(x, S(x)) \leq 0$ for all $x \in \mathbb{R}$.

and $f_{s}(x, \cdot)$ satisfies this uniform Lipschitz condition 
(H5)

$$
\sup _{x \in \mathbb{R}}\left(\sup _{s_{0}, s_{1} \geq 0} \frac{\left|f_{s}\left(x, s_{0}\right)-f_{s}\left(x, s_{1}\right)\right|}{\left|s_{0}-s_{1}\right|}\right)<+\infty
$$

A typical example of such a non linearity is given by $f(x, s):=s(a(x)-b(x) s)$ with $b(x)>0, b \in L^{\infty}$ and $a(x)$ satisfying $\lim \sup _{|z| \rightarrow \infty} a(z)<0$. This structure of the environment prohibits the existence of monotone fronts and the analysis provided in [42] no longer holds true.

This type of moving environments were recently investigated in 32] for a very specific type of nonlinearity $f(x, s)$ presenting some symmetry. Namely, assuming that $f(x, s)$ satisfies the conditions:

$$
\left\{\begin{array}{l}
f \in C^{1}\left(\mathbb{R}^{2}\right) \text { and there are } a, q, L, L_{0}>0 \text { and } \phi^{+}, \phi^{-} \in C^{1}(\mathbb{R}) \text { such that } \\
\forall s, \forall|x| \geq L+L_{0}, f(x, s)=-q s \\
\forall s, \forall|x| \leq L, f(x, s)=s(a-s) \\
\forall s, \forall L \leq x \leq L+L_{0}, f(x, s)=-q s+s[a-s+q] \phi^{+}\left(\frac{x-L}{L_{0}}\right) \\
\forall s, \forall-L-L_{0} \leq x \leq-L, f(x, s)=-q s+s[a-s+q] \phi^{-}\left(\frac{x-L}{L_{0}}\right)
\end{array}\right.
$$

where $\phi^{+}$and $\phi^{-}$are respectively smooth regularisation of the characteristic function of $\mathbb{R}^{-}$and of $\mathbb{R}^{+}$, such that $\operatorname{supp}\left(\phi^{+}\right) \subset(-\infty, 1), \phi_{\mid \mathbb{R}^{-}}^{+} \equiv 1$ and $\phi^{-}(x)=\phi^{+}(-x)$.

For such specific nonlinearities and assuming that $J$ is thin tailed and symmetric, the authors in [32] prove the existence of critical speed $c^{*}>0$ determined by the maximal linearised growth rate (i.e. $c^{*}$ defined by (1.3) with $\sup _{x \in \mathbb{R}} r(x)=a$ ) such that for all $c \geq c^{*}$ the population go extinct whereas for $0<c<c^{*}$, the population persistence is dependant of the patch size habitat $L$, i.e., the population survive if the patch size is greater that some critical value $L^{*}$ otherwise the population dies. To obtain these results the authors rely on some notion of persistence for the solution $u(t, x)$ of the Cauchy problem $(1.2)$, namely the population is said to persist if for any initial datum $u_{0}$ such that $\inf _{\mathbb{R}} u_{0}>0$, then the corresponding solution of the Cauchy problem (1.2) with initial data $u_{0}$ satisfies for all compact $K \subset \mathbb{R}$,

$$
\liminf _{t \rightarrow+\infty} \inf _{x-c t \in K} u(t, x)>0 .
$$

Our main purpose here is to extend the above results to more general situations by finding more generic conditions on $J, c$ and $f$ that characterise the persistence of the species modelled by (1.2).

In this task, as in [32, 42] we focus our analysis on the description of positive (1.2) defined in a moving frame of speed $c$. That is we look for solution $\tilde{U}(t, x):=U(t, x+c t)$ that satisfies

$$
\partial_{t} \tilde{U}(t, \xi)-c \mathfrak{D}_{\xi}[\tilde{U}](t, \xi)=\int_{\mathbb{R}} J\left(\xi-\xi^{\prime}\right) \tilde{U}\left(t, \xi^{\prime}\right) d y-\tilde{U}(t, \xi)+f(\xi, \tilde{U}(t, \xi)) \quad \text { for } \quad t>0, \xi \in \mathbb{R}
$$

where we set $\xi:=x+c t$ as a new variable and we use the Euler's notation to denote differential operators $\frac{d}{d x} f(x)$, i.e. $\mathfrak{D}_{x}[f]=\frac{d}{d x} f(x)$. In particular, we look for stationary solutions $u$ of (1.4) which are then positive solutions of the equation below

$$
-c \mathfrak{D}_{x}[u](x)=J \star u(x)-u(x)+f(x, u(x)) \quad \text { for } \quad x \in \mathbb{R},
$$

Existence of such stationary solutions is naturally expected to provide the right persistence criterion. We will see that this is indeed the case.

In the literature, such problem have been well studied for the local reaction diffusion version of (1.2)

$$
\partial_{t} U(t, z)=\Delta U(t, z)+f\left(z_{1}-c t, y, U(t, z)\right) \text { for } t>0, \text { and } z \in \Omega,
$$

where $\Omega$ is cylindrical domain of $\mathbb{R}^{N}$, possibly $\mathbb{R}^{N}$ itself see for example [13, 6, 14, 56. For such reaction diffusion equations the persistence criteria are often derived from the sign of the first eigenvalue of the linearised problem at the 0 solution. One is thus led to determine the sign of the first eigenvalue $\lambda_{1}(\Delta+$ $\left.c \partial_{x_{1}}+\partial_{s} f\left(x_{1}, y, 0\right), \Omega\right)$ of the spectral problem

$$
\Delta \varphi+c \partial_{x_{1}} \varphi+\partial_{s} f\left(x_{1}, y, 0\right) \varphi+\lambda_{1} \varphi=0 \quad \text { in } \quad \Omega
$$

associated with the proper boundary conditions (if $\Omega \neq \mathbb{R}^{N}$ ). 
In the above situation the existence of a positive stationary solution to (1.6) is uniquely conditioned by the sign of $\lambda_{1}$. More precisely, there exists a unique positive stationary solution if and only if $\lambda_{1}<0$. If such type of criteria seems reasonable for problems defined on bounded set, it is less obvious for problems in unbounded domains. In particular, in unbounded domains, one of the main difficulty concerns the definition of $\lambda_{1}$. As shown in [12, 8, 15, the notion of first eigenvalue in unbounded domain can be quite delicate and several definitions of $\lambda_{1}$ exist rendering the question of sharp persistence criteria already quite involved. When such existence criteria is established, it is then possible to investigate its behaviour with respect to the speed $c$ and for the above problem, using the Liouville transform, it is easy to make explicit the dependence of $\lambda_{1}$ with respect to $c$. Indeed, in such situation we have

$$
\lambda_{1}\left(\Delta+c \partial_{x_{1}}+\partial_{s} f\left(x_{1}, y, 0\right)\right)=\lambda_{1}\left(\Delta+\partial_{s} f\left(x_{1}, y, 0\right)\right)+\frac{c^{2}}{4} .
$$

From the above formula, it is then easy to derive the critical speed for which a species can survive.

Much less is known for the non-local equation (1.5) and, persistence criteria have been essentially investigated in some specific situations such as periodic media : [26, 28, 53, 49, 52] or for a version of the problem (1.5) with no time dependence [3, 5, 24, 25, 34, 41, 53] :

$$
\partial_{t} U(t, x)=\int_{\Omega} J(x-y) U(t, y) d y-U(t, x)+f(x, U(t, x)) \quad \text { for } t>0, \text { and } x \in \Omega .
$$

Similarly to the local diffusion case, for KPP like non-linearities, the existence of a positive solution of the non-local equation (1.8) can be characterised by the sign of a spectral quantity $\lambda_{p}$, called the generalised principal eigenvalue or the spectral point of

$$
\int_{\Omega} J(x-y) \varphi(y) d y-\varphi+\partial_{s} f(x, 0) \varphi+\lambda \varphi=0 \quad \text { in } \quad \Omega .
$$

In the spirit of [11, this generalised principal eigenvalue $\lambda_{p}$ can be defined by :

$$
\lambda_{p}:=\sup \left\{\lambda \in \mathbb{R} \mid \exists \varphi \in C(\Omega), \varphi>0, \text { such that } \mathcal{M}_{\Omega}[\varphi]+\partial_{s} f(x, 0) \varphi+\lambda \varphi \leq 0 \text { in } \Omega\right\},
$$

where $\mathcal{M}_{\Omega}[\varphi]$ denotes

$$
\mathcal{M}_{\Omega}[\varphi]:=\int_{\Omega} J(x-y) \varphi(y) d y-\varphi .
$$

This is only very recently that some progress have been made on the spectral theory of nonlocal operators with a drift term, that is operators of the type $c \mathfrak{D}_{x}+\mathcal{M}_{\Omega}+\mathbf{a}$ see for example [1, 31, 30, 32, 42, 55]. This new understanding of such spectral problems provide an adequate framework to the analysis of for complex media such as those described in [42] or those we study in the present paper. As in [5, 31, 30, for the operator $c \mathfrak{D}_{x}+\mathcal{M}_{\Omega}+\mathbf{a}$ let us define the quantity

$\lambda_{p}\left(c \mathfrak{D}_{x}+\mathcal{M}_{\Omega}+\mathbf{a}\right):=\sup \left\{\lambda \in \mathbb{R} \mid \exists \varphi \in C^{1}\left(\mathbb{R}^{N}\right), \varphi>0\right.$ such that $\left.c \mathfrak{D}_{x}[\varphi]+\mathcal{M}_{\Omega}[\varphi]+a(x) \varphi+\lambda \varphi \leq 0\right\}$. Equipped with this notion, we can now state our main results. In the first one we establish a simple sharp persistence criteria assuming that the dispersal kernel $J$ has a compact support.

Theorem 1.1. Assume that $J, f$ satisfy ( and $c>0$. Then, there exists a positive solution, $u$, of (1.5) if and only if $\lambda_{p}\left(c \mathfrak{D}_{x}+\mathcal{M}+\partial_{\mathbf{s}} \mathbf{f}(\mathbf{x}, \mathbf{0})\right)<0$, where $\mathcal{M}$ denotes the continuous operator $\mathcal{M}[\varphi]=J \star \varphi-\varphi$ and

$\lambda_{p}\left(c \mathfrak{D}_{x}+\mathcal{M}+\partial_{\mathbf{s}} \mathbf{f}(\mathbf{x}, \mathbf{0})\right):=\sup \left\{\lambda \in \mathbb{R} \mid \exists \varphi \in C^{1}(\mathbb{R}), \varphi>0\right.$ such that $\left.c \mathfrak{D}_{x}[\varphi]+\mathcal{M}[\varphi]+\partial_{s} f(x, 0) \varphi+\lambda \varphi \leq 0\right\}$. When it exists, the solution is unique, that is, if $v$ is another bounded solution, then $u=v$ almost everywhere. Moreover, for any non-negative initial data $u_{0} \in C(\mathbb{R}) \cap L^{\infty}(\mathbb{R})$ we have the following asymptotic behaviour:

- If $\lambda_{p}\left(c \mathfrak{D}_{x}+\mathcal{M}+\partial_{s} f(x, 0)\right) \geq 0$, then the solution satisfies $\|U(t, \cdot)\|_{\infty} \rightarrow 0$ as $t \rightarrow \infty$,

- If $\lambda_{p}\left(c \mathfrak{D}_{x}+\mathcal{M}+\partial_{s} f(x, 0)\right)<0$, then the solution satisfies $\|U(t, \cdot)-u(\cdot-c t)\|_{\infty} \rightarrow 0$ as $t \rightarrow \infty$.

Next, we establish some properties of the quantity $\lambda_{p}\left(c \mathfrak{D}_{x}+\mathcal{M}+\partial_{\mathbf{s}} \mathbf{f}(\mathbf{x}, \mathbf{0})\right)$ that will help to analyse the dependence of $\lambda_{p}$ with respect to the speed $c$. Namely, 
Theorem 1.2. Assume that $J, f$ satisfy $(\overline{\mathbf{H 1}})-(\overline{\mathbf{H} 4})$ and assume further that $J$ is compactly supported. Then the map

$$
\begin{array}{lll}
\mathbb{R}^{+} & \rightarrow \mathbb{R} \\
c & \mapsto \lambda_{p}\left(c \mathfrak{D}_{x}+\mathcal{M}+\partial_{\mathbf{s}} \mathbf{f}(\mathbf{x}, \mathbf{0})\right)
\end{array}
$$

is continuous. In addition, we have

$$
\lambda_{p}\left(c \mathfrak{D}_{x}+\mathcal{M}+\partial_{\mathbf{s}} \mathbf{f}(\mathbf{x}, \mathbf{0})\right)=\lambda_{p}\left(-c \mathfrak{D}_{x}+\mathcal{M}^{*}+\partial_{\mathbf{s}} \mathbf{f}(\mathbf{x}, \mathbf{0})\right)
$$

and

$$
\lambda_{p}\left(c \mathfrak{D}_{x}+\mathcal{M}+\partial_{\mathbf{s}} \mathbf{f}(\mathbf{x}, \mathbf{0})\right)=\lambda_{p}\left(-c \mathfrak{D}_{x}+\mathcal{M}^{*}+\partial_{\mathbf{s}} \mathbf{f}(-\mathbf{x}, \mathbf{0})\right)
$$

where $\mathcal{M}^{*}$ is the dual operator of $\mathcal{M}$ that is

$$
\mathcal{M}^{*}[\varphi]:=\int_{\mathbb{R}} J(y-x) \varphi(y) d y-\varphi(x) .
$$

With this two results, we can have a first description of the effect of the speed $c$ on the persistence of the species. More precisely, we can show that

Theorem 1.3. Let $f, J$ be as in Theorem 1.1 and assume that $\lambda_{p}\left(\mathcal{M}+\partial_{\mathbf{s}} \mathbf{f}(\mathbf{x}, \mathbf{0})\right)<0$. Then there exists $0<c^{*,+}<c^{* *,+}$ and $0<c^{*,-}<c^{* *,-}$ such that for all $-c^{*,-}<c<c^{*,+}$ there exists a positive solution to (1.5) whereas none exists when $c \geq c^{* *,+}$ or $c \leq-c^{* *,-}$.

The existence of such critical speed $c^{*, \pm}$ and $c^{* *, \pm}$ comes as a corollary of the sharp existence criteria obtained in Theorem 1.1 and the study of the behaviour of $\lambda_{p}\left(c \mathfrak{D}_{x}+\mathcal{M}+\partial_{\mathbf{s}} \mathbf{f}(\mathbf{x}, \mathbf{0})\right)$ with respect to the speed $c>0$, in particular its continuity and the equality $\lambda_{p}\left(c \mathfrak{D}_{x}+\mathcal{M}+\partial_{\mathbf{s}} \mathbf{f}(\mathbf{x}, \mathbf{0})\right)=\lambda_{p}\left(-c \mathfrak{D}_{x}+\mathcal{M}^{*}+\right.$ $\left.\partial_{\mathbf{s}} \mathbf{f}(\mathbf{x}, \mathbf{0})\right)$ (Theorem 1.2).

When $J$ is symmetric, we have a more simple description of the critical speed $c^{*,+}, c^{*,-}, c^{* *,+}$ and $c^{* *,-}$. Indeed, in this situation we show that $c^{*,+}=c^{*,-}$ and $c^{* *,+}=c^{* *,-}$ and therefore we have

Theorem 1.4. Let $f, J$ be as in Theorem 1.1 and assume further that $J$ is symmetric and that $\lambda_{p}(\mathcal{M}+$ $\left.\partial_{\mathbf{s}} \mathbf{f}(\mathbf{x}, \mathbf{0})\right)<0$. Then there exists $0<c^{*}<c^{* *}$ such that for all $|c|<c^{*}$ there exists a positive solution to (1.5) whereas none exists when $|c| \geq c^{* *}$.

This results is a direct consequence of Theorem 1.2, as for symmetric $J$, we then have

$$
\lambda_{p}\left(c \mathfrak{D}_{x}+\mathcal{M}+\partial_{\mathbf{s}} \mathbf{f}(\mathbf{x}, \mathbf{0})\right)=\lambda_{p}\left(-c \mathfrak{D}_{x}+\mathcal{M}+\partial_{\mathbf{s}} \mathbf{f}(\mathbf{x}, \mathbf{0})\right) .
$$

Note that due to possible non symmetrical structure of the nonlinearity we might have expect that the critical speed $c^{*}, c^{* *}$ depends in some ways on the sign of the speed of environmental change. This is surprisingly not the case, meaning that there is no qualitative difference on the survival of the population when the niche move to the right $(c>0)$ or to the left $(c<0)$, when the dispersal process is symmetric. In this situation the critical speeds $c^{*}, c^{* *}$ are uniquely determined independently from the sign of the speed $c$. The existence of a preferred direction for survival is uniquely determine by the symmetry of dispersal process.

The question of sharp thresholds for the speeds, i.e. $c^{*}=c^{* *}, c^{*,+}=c^{* *,+}$ and $c^{*,-}=c^{* *,-}$ as well as the existence of formulas describing them as in the classical case are still open problems which seems intimately related to the properties of the kernel $J$. However, as in [32], we can have a first estimate of $c^{* *, \pm}$. Namely we have

Proposition 1.5. Let $f, J$ be as in Theorem 1.1 and assume that $\lambda_{p}\left(\mathcal{M}+\partial_{\mathbf{s}} \mathbf{f}(\mathbf{x}, \mathbf{0})\right)<0$. Then

$$
\begin{gathered}
c^{* *,+} \leq c_{\alpha}^{+}:=\inf _{\alpha>0} \frac{1}{\alpha}\left(\int_{\mathbb{R}} J(z) e^{\alpha z} d z-1+\sup _{x \in \mathbb{R}} f_{s}(x, 0)\right) \\
c^{* *,-} \leq c_{\alpha}^{-}:=\inf _{\alpha>0} \frac{1}{\alpha}\left(\int_{\mathbb{R}} J(-z) e^{\alpha z} d z-1+\sup _{x \in \mathbb{R}} f_{s}(x, 0)\right)
\end{gathered}
$$


Last we investigate the effect of the tail of the kernel on the survival of the population. To simplify our analysis we restrict our analysis to situations where $\sup _{\mathbb{R}} \partial_{s} f(x, 0)>1$ and $J$ is symmetric and satisfies a second moment condition, that is

$$
\int_{\mathbb{R}} J(z)|z|^{2} d z<+\infty
$$

For such kernels and nonlinearities, we can extend the previous result of Theorem 1.3. Namely, we have

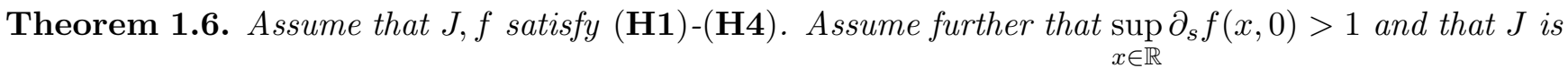
symmetric and satisfies (프). Then, there exists Then there exists $0<c^{*}<c^{* *}$ such that for all $|c|<c^{*}$ there exists a positive solution to (1.5) whereas none exists when $|c| \geq c^{* *}$.

Observe that the above results is a strong indication on robustness of our results with respect to the tail of the kernel, in the sense that even for a fat-tailed kernel, for example $J(z) \sim \frac{1}{|z|^{4}}$, we observe the same pattern for the critical speed. In particular, it highlights the fact that even population having a very strong spreading capabilities at a large scale can died out in a shifting environment.

1.1. Further comments. Before going to the proof of these results, we would like to make some further comments on the results obtained and explain our main strategy to prove them.

First, due to the particular structure of this problem, it is expected that the properties of the principal eigenvalue $\lambda_{p}\left(c \mathfrak{D}_{x}+\mathcal{M}+\partial_{\mathbf{s}} \mathbf{f}(\mathbf{x}, \mathbf{0})\right)$ with respect to all its parameters will play a key role in the proofs. This fact is also present in [32] where we can see the important role played by the linearised operator around the trivial solution both in the definition of the critical speed and in the construction of a non trivial solution. However, althougth we can find in 32] some elements related to the spectral theory of the operator $c \mathfrak{D}_{x}+\mathcal{M}+\partial_{\mathbf{s}} \mathbf{f}(\mathbf{x}, \mathbf{0})$ defined for particular structure of the zero order term, there is no clear spectral theory defined for a general operators $c \mathfrak{D}_{x}+\mathcal{M}+\partial_{\mathbf{s}} \mathbf{f}(\mathbf{x}, \mathbf{0})$ whose zero order term is just assumed bounded. Through the definition we give of $\lambda_{p}\left(c \mathfrak{D}_{x}+\mathcal{M}+\mathbf{a}\right)$ and our analysis of its properties, we provide a clear and acurate way for the description of the first fundamental element of the spectrum of these type of operators.

A great deal of the analysis presented here, provides a clear description of this spectral quantity and establish some of its main properties. We believe that these fundamental results will be also of some help to understand other situation in particular in evolutionary biology [16, 50.

Second, we would also emphasize that our result do not require any specific symmetry for the nonlinearity as well for the kernel $J$, properties that are strongly used in [32] to derive their persistence criteria. Although our results mostly concerns compactly supported kernels, our existence criteria apply thereby on a larger variety of possible non-linearity $f$ and $J$. In particular, we would like to highlight that as in the analysis of the case $c=0$ given in [5, 18]), our result on fat tailed kernel shows that the assumption on support of the kernel $J$ we made is technical and is not a prerequisite for analysing such type of problem. We found that the main difficulty in the analysis such situation relies on the lack of adequate tools, in particular the lack of an accurate spectral theory for operator involving such kernels. Recent progress in the understanding of spectral properties of such operators have been recently obtained in [22, 55] using probabilistic methods. Wit this respect, with the proper spectral theory, we believe that our results should hold true for a fractional version of (1.5) where the operator $J \star \varphi-\varphi$ is replaced by the Fractional Laplacian $\Delta^{s} \varphi$.

Being at the core the paper, let us briefly explain our main strategy to construct a positive non-trivial solution of (1.5). To construct such a nontrivial solution we use the vanishing viscosity approach, approach previously used in this context for example in [29, 23, 27]. The main idea is to introduce the following regularised problem :

$$
\varepsilon \mathfrak{D}_{x x}[u](x)+c \mathfrak{D}_{x}[u](x)+\mathcal{M}[u](x)+f(x, u(x))=0 \quad \text { for } \quad x \in \mathbb{R},
$$


and to show that for a fixed $c>0$ such that $\lambda_{p}\left(c \mathfrak{D}_{x}+\mathcal{M}+\mathbf{a}\right)<0$ we can find $\varepsilon_{0}(c)>0$ such that for $0<\varepsilon \leq \varepsilon_{0}$, the problem (4.1) admits a unique positive non trivial solution. That is, to prove the following statement

Theorem 1.7. Assume that $f$ and $J$ satisfy $(\overline{\mathbf{H 1}})-(\underline{\mathbf{H} 4})$ and that $c>0$ is such that $\lambda_{p}\left(c \mathfrak{D}_{x}+\mathcal{M}+\right.$ $\left.\partial_{s} f(x, 0)\right)<0$. Then there exists $\varepsilon_{0}>0$ such that for all $0<\varepsilon \leq \varepsilon_{0}$ there exists a unique positive smooth function, $u_{\varepsilon}$, solution to (1.10).

From this result, we can then obtain a solution of (1.5), by studying the singular limit problem when $\varepsilon \rightarrow 0$. The proof of Theorem 1.7 crucially relies on the properties of some spectral quantities. We prove in particular that for any $\Omega \subset \mathbb{R}$ domain we can define

$$
\begin{array}{r}
\lambda_{p}\left(\varepsilon \mathfrak{D}_{x x}+c \mathfrak{D}_{x}+\mathcal{M}_{\Omega}+\mathbf{a}\right):=\sup \left\{\lambda \in \mathbb{R} \mid \exists \varphi \in C^{0}(\bar{\Omega}) \cap C^{2}(\Omega), \varphi>0\right. \\
\text { such that } \left.\varepsilon \mathfrak{D}_{x x}[\varphi]+c \mathfrak{D}_{x}[\varphi]+\mathcal{M}_{\Omega}[\varphi]+a(x) \varphi+\lambda \varphi \leq 0\right\} .
\end{array}
$$

and show that for any compact domain $\Omega$ we have

$$
\lim _{\varepsilon \rightarrow 0} \lambda_{p}\left(\varepsilon \mathfrak{D}_{x x}+c \mathfrak{D}_{x}+\mathcal{M}_{\Omega}+\mathbf{a}\right)=\lambda_{p}\left(c \mathfrak{D}_{x}+\mathcal{M}_{\Omega}+\mathbf{a}\right)
$$

The paper is organised as follows. In Section 2 we prove some useful comparison principle in unbounded domain. Then in Section 3, we recall some basic theory on the principal eigenvalue $\lambda_{p}\left(c \mathfrak{D}_{x}+\mathcal{M}_{\Omega}+\mathbf{a}\right)$ and study several of its properties. Within this section we analyse in particular the dependence of this principal eigenvalue with respect to the speed $c$ (Theorem 1.2) and prove Theorem 1.3 assuming that the sharp existence criteria of Theorem 1.1 is true. In Section 4 we obtained existence and uniqueness of the regularised problem (1.10) and prove Theorem [1.7. In Sections 56 and 7 we prove the sharp existence criteria stated in Theorem 1.1, by proving successively, the sufficient condition for the existence of a stationary solution (5), the uniqueness of the stationary solution when it exists (6) , and at last the necessary condition (77). The analysis of the long time behaviour is made in the last section, Section 8 , concluding the proof of Theorem 1.1. Finally, in the last section, Section 9, we analyse the fat tailed case and prove Theorem 1.6 .

1.2. Notations. To simplify the presentation we introduce some notations and various linear operator that we will use throughout this paper:

- We denote by $\mathcal{M}_{\Omega}$ the continuous linear operator

$$
\begin{aligned}
\mathcal{M}_{\Omega}: C(\bar{\Omega}) & \rightarrow C(\bar{\Omega}) \\
u & \mapsto \int_{\Omega} J(x-y) u(y) d y-u,
\end{aligned}
$$

where $\Omega \subset \mathbb{R}$.

- $\mathcal{M}_{R}$ corresponds to the continuous operator $\mathcal{M}_{\Omega}$ with $\Omega=(-R, R)$,

- We will use $\mathcal{M}$ to denote the operators $\mathcal{M}_{\Omega}$ with $\Omega=\mathbb{R}$.

- We will use also Euler's notation to denote differential operators namely $\mathfrak{D}_{x}$ and $\mathfrak{D}_{x x}$ will denote respectively the following differential operator $\mathfrak{D}_{x}[f]=\frac{d}{d x} f(x)$ and $\mathfrak{D}_{x x}[f]:=\frac{d^{2}}{d x^{2}} f(x)$.

\section{COMPARISON PRINCIPLES}

In this section we collect comparison principles that fit for our purposes. Let us start by proving a weak comparison principle that holds when $a$ is negative outside a compact. Namely, we let us prove the following

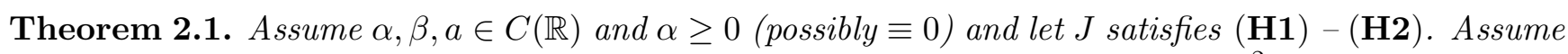
further there exists $R_{0}>0$ such that $a(x) \leq 0$ for all $|x| \geq R_{0}$. Let $u, v \in C(\mathbb{R}) \cap C_{\text {loc }}^{2}(\mathbb{R})$ be such that

$$
\left\{\begin{array}{l}
\alpha(x) \mathfrak{D}_{x x}[u](x)+\beta(x) \mathfrak{D}_{x}[u](x)+\mathcal{M}[u](x)+a(x) u(x) \leq 0 \quad \text { for all } \quad|x| \geq R_{0} \\
\alpha(x) \mathfrak{D}_{x x}[v](x)+\beta(x) \mathfrak{D}_{x}[v](x)+\mathcal{M}[v](x)+a(x) v(x) \geq 0 \quad \text { for all } \quad|x| \geq R_{0} \\
\lim _{|x| \rightarrow+\infty} u(x) \geq \lim _{|x| \rightarrow+\infty} v(x)=0 \\
v(x) \leq u(x) \quad \text { for all } \quad|x| \leq R_{0}
\end{array}\right.
$$

then $v(x) \leq u(x)$ for all $x \in \mathbb{R}$. 
Proof. The proof is rather elementary but for the sake of completeness we include all the details. By definition of $a$ we can check that for every $\delta>0$ we have

$$
\begin{array}{ll}
\alpha(x) \mathfrak{D}_{x x}[u+\delta](x)+\beta(x) \mathfrak{D}_{x}[u+\delta](x)+\mathcal{M}[u+\delta](x)+a(x)(u(x)+\delta)<0 & \text { for all }|x|>R_{0}, \\
\alpha(x) \mathfrak{D}_{x x}[v](x)+\beta(x) \mathfrak{D}_{x}[v](x)+\mathcal{M}[v](x)+a(x) v(x) \geq 0 & \text { for all }|x|>R_{0}, \\
v(x)<u(x)+\delta & \text { for all }|x| \leq R_{0} .
\end{array}
$$

In addition, since $v \leq u$ as $|x| \rightarrow+\infty$ there exists $R_{\delta}>R_{0}$ such that $v(x)<u(x)+\delta$ for all $|x| \geq R_{\delta}$. From there we then see that $v \leq u+\delta$ in $\mathbb{R}$. Indeed, if not then $\sup _{x \in \mathbb{R}}(v(x)-u(x)-\delta)>0$ and since $v<u+\delta$ in $\left[-R_{0}, R_{0}\right] \cup\left(\mathbb{R} \backslash\left(-R_{\delta}, R_{\delta}\right)\right)$ we have

$$
\sup _{\mathbb{R}}(v(x)-u(x)-\delta)=\max _{R_{\delta}<|x|<R_{0}}(v(x)-u(x)-\delta) .
$$

Let $x_{0} \in\left(-R_{\delta}, R_{\delta}\right) \backslash\left[-R_{0}, R_{0}\right]$ be the point where the function $v-u-\delta$ achieved its maximum, then we have $\mathfrak{D}_{x}[v-u-\delta]\left(x_{0}\right)=0$ and $\mathfrak{D}_{x x}[v-u-\delta]\left(x_{0}\right) \leq 0, \mathcal{M}[v-u-\delta]\left(x_{0}\right) \leq 0, a\left(x_{0}\right)\left(v\left(x_{0}\right)-u\left(x_{0}\right)-\delta\right) \leq 0$ and by evaluating (2.1) and (2.2) at this point we also get

$$
\begin{aligned}
0 & \leq \alpha\left(x_{0}\right) \mathfrak{D}_{x x}[v-u-\delta]\left(x_{0}\right)+\beta\left(x_{0}\right) \mathfrak{D}_{x}[v-u-\delta]\left(x_{0}\right)+\mathcal{M}[v-u-\delta]\left(x_{0}\right)+a\left(x_{0}\right)\left(v\left(x_{0}\right)-u\left(x_{0}\right)-\delta\right) \\
& =\alpha\left(x_{0}\right) \mathfrak{D}_{x x}[v-u-\delta]\left(x_{0}\right)+\mathcal{M}[v-u-\delta]\left(x_{0}\right)+a\left(x_{0}\right)\left(v\left(x_{0}\right)-u\left(x_{0}\right)-\delta\right) \leq 0 .
\end{aligned}
$$

Therefore $\mathcal{M}[v-u-\delta]\left(x_{0}\right)=0$ and we must have $v(x)-u(x)-\delta=v\left(x_{0}\right)-u\left(x_{0}\right)-\delta$ for all $x \in$ $x_{0}+\operatorname{supp}(J)$. By redoing the above argument with any point of $x_{0}+\operatorname{supp}(J)$, we then see that $v(x)-$ $u(x)-\delta=v\left(x_{0}\right)-u\left(x_{0}\right)-\delta$ for all $x \in x_{0}+2 \cdot \operatorname{supp}(J)$ and by arguing inductively we can then check that for all $n \in \mathbb{N}, v(x)-u(x)-\delta=v\left(x_{0}\right)-u\left(x_{0}\right)-\delta$ for all $x \in x_{0}+n \cdot \operatorname{supp}(J)$. Now since $J$ satisfies (H2), i.e. $J(0)>0$, then $\lim _{n \rightarrow \infty} x_{0}+n \cdot \operatorname{supp}(J)=\mathbb{R}$ and thus we deduce the following contradiction

$$
-\delta=\lim _{|x| \rightarrow+\infty} v(x)-u(x)-\delta=v\left(x_{0}\right)-u\left(x_{0}\right)-\delta>0 .
$$

Whence $v \leq u+\delta$. The previous argumentation holding true for all $\delta \geq 0$, we then conclude that

$$
v(x) \leq u(x) \text { for all } \quad x \in \mathbb{R} .
$$

Last let us recall a classical strong comparison principle

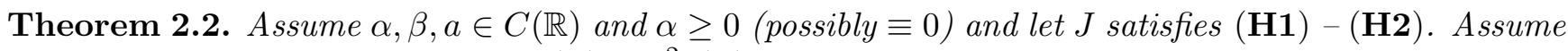
further that $a \in L^{\infty}$ and let $u, v \in C(\mathbb{R}) \cap C_{\text {loc }}^{2}(\mathbb{R})$ be such that

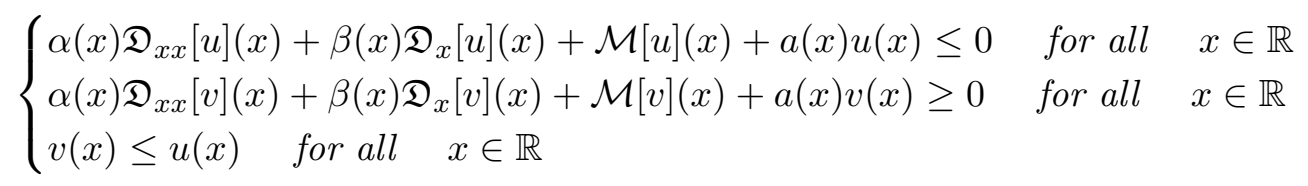

then either $v \equiv u$ or $v(x)<u(x)$ for all $x \in \mathbb{R}$.

Proof. The proof is rather standard. Since $a \in L^{\infty}$ there exists $k>0$ such that $a(x)-k \leq 0$ and thus the non negative function $z:=u-v$ satisfies

$$
\alpha(x) \mathfrak{D}_{x x}[z](x)+\beta(x) \mathfrak{D}_{x}[z](x)+\mathcal{M}[z](x)+a(x) z(x) \leq 0 \quad \text { for all } \quad x \in \mathbb{R} .
$$

Now it is there standard to see that either $z \equiv 0$ or $z>0$. Indeed, if there exists $x_{0}$ such that $0=z\left(x_{0}\right)=$ $\min _{x \in \mathbb{R}} z(x)$ then we get

$$
0 \leq \int_{\mathbb{R}} J\left(x_{0}-y\right) z(y) d y \leq 0,
$$

and by a classical argument we get $z \equiv 0$. So $v \equiv u$. Otherwise we have $z>0$ and so $u>v$.

Remark 1. Note that the above proofs only relies on the properties of the operator $\mathcal{M}$ and so the Theorems are still true if $\alpha(x) \equiv 0$ and/or $\beta \equiv 0$. In such situation, the $C_{l o c}^{2}$ regularity for $u$ and $v$ is not needed and we can only require that $u, v \in C_{\text {loc }}^{1}(\mathbb{R})$ or $C(\mathbb{R})$ if also $\beta \equiv 0$. 


\section{Spectral Theory of integro-differential operators}

In this section, we recall some important results on the principal eigenvalue of the linear non-local operator $c \mathfrak{D}_{x}+\mathcal{M}_{\Omega}+\mathbf{a}$ and derive some new properties of this quantity, in particular its behaviour with respect to the parameter $c$. We also recall some known variational characterisation of the principal eigenvalue of general integrodifferential operators. We split this section in two parts, one dealing with the spectral properties of the principal eigenvalue $\lambda_{p}$ defined for nonlocal operators with a drift and a second recalling some elements of the spectral theory regarding integrodifferential operators containing an elliptic part.

3.1. Principal eigenvalue for non-local operators with a drift. In this subsection, we recall some results on the principal eigenvalue of a linear non-local operator $c \mathfrak{D}_{x}+\mathcal{M}_{\Omega}+\mathbf{a}$ and establish new ones. That is, we focus on the properties of the spectral problem

$$
c \mathfrak{D}_{x}[\varphi]+\mathcal{M}_{\Omega}[\varphi]+a(x) \varphi+\lambda \varphi=0 \quad \text { in } \quad \Omega .
$$

Following Berestycki, Nirenberg and Varadhan [1], we define the principal eigenvalue $\lambda_{p}$ the following way,

$$
\lambda_{p}=\sup \left\{\lambda \mid \exists \varphi \in C^{1}(\Omega), \varphi>0, c \mathfrak{D}_{x}[\varphi]+\mathcal{M}_{\Omega}[\varphi]+a(x) \varphi+\lambda \varphi \leq 0\right\} .
$$

When $c=0$, then $C^{1}(\Omega)$ the set of test functions is replaced by $C(\Omega)$.

3.1.1. Generic Properties. For such $\lambda_{p}$, let us first recall some standard properties that we constantly use throughout this paper:

Proposition 3.1. $\quad$ (i) Assume $\Omega_{1} \subset \Omega_{2}$, then for the two operators $c \mathfrak{D}_{x}+\mathcal{M}_{\Omega_{1}}+\mathbf{a}$ and $c \mathfrak{D}_{x}+\mathcal{M}_{\Omega_{2}}+\mathbf{a}$ respectively defined on $C^{1}\left(\Omega_{1}\right)$ and $C^{1}\left(\Omega_{2}\right)$, we have :

$$
\lambda_{p}\left(c \mathfrak{D}_{x}+\mathcal{M}_{\Omega_{1}}+\mathbf{a}\right) \geq \lambda_{p}\left(c \mathfrak{D}_{x}+\mathcal{M}_{\Omega_{2}}+\mathbf{a}\right) .
$$

(ii) For a fixed $\Omega$ and assume that $a_{1}(x) \geq a_{2}(x)$, for all $x \in \Omega$. Then

$$
\lambda_{p}\left(c \mathfrak{D}_{x}+\mathcal{M}_{\Omega}+\mathbf{a}_{2}\right) \geq \lambda_{p}\left(c \mathfrak{D}_{x}+\mathcal{M}_{\Omega}+\mathbf{a}_{\mathbf{1}}\right) .
$$

(iii) $\lambda_{p}\left(c \mathfrak{D}_{x}+\mathcal{M}_{\Omega}+\mathbf{a}\right)$ is Lipschitz continuous with respect to a. More precisely,

$$
\left|\lambda_{p}\left(c \mathfrak{D}_{x}+\mathcal{M}_{\Omega}+\mathbf{a}\right)-\lambda_{p}\left(c \mathfrak{D}_{x}+\mathcal{M}_{\Omega}+\mathbf{b}\right)\right| \leq\|a-b\|_{\infty}
$$

(iv) For any $\Omega \subset \mathbb{R}$, we always have the following bounds

$$
\lambda_{p}\left(c \mathfrak{D}_{x}+\mathcal{M}_{\Omega}+\mathbf{a}\right) \geq-\sup _{x \in \Omega}\left(\mathcal{M}_{\Omega}[1](x)+a(x)\right) .
$$

(v) $\lambda_{p}\left(\mathcal{M}_{\Omega}+\mathbf{a}\right)$ is continuous with respect to $J$.

(vi) $\lambda_{p}\left(c \mathfrak{D}_{x}+\mathcal{M}_{\Omega}+\mathbf{a}\right)$ is continuous with respect to $c$.

The proofs are rather standard and essentially use the definition of $\lambda_{p}$. We point to [4, 24, 25] for the proofs of $(i)-(i v)$ in the situation where $c=0$ and to [30] when $c \neq 0$.

Let us now state two important properties of the principal eigenvalue. The first one is a Collatz-Wieland type characterization of $\lambda_{p}$. Namely,

Theorem 3.2. Assume that $\Omega=\left(r_{1}, r_{2}\right) \subset \mathbb{R}$ (with $\left.r_{1}<r_{2}\right)$ is a bounded domain and let a, $J$ such that

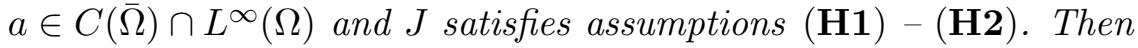

$$
\lambda_{p}\left(c \mathfrak{D}_{x}+\mathcal{M}_{\Omega}+\mathbf{a}\right)=\tilde{\lambda}_{p}^{\prime}\left(c \mathfrak{D}_{x}+\mathcal{M}_{\Omega}+\mathbf{a}\right),
$$

where for $c>0$

$$
\tilde{\lambda}_{p}^{\prime}\left(c \mathfrak{D}_{x}+\mathcal{M}_{\Omega}+\mathbf{a}\right)=\inf \left\{\lambda \mid \exists \varphi \in C^{1}(\Omega) \cap C(\bar{\Omega}), \varphi>0, \varphi\left(r_{2}\right)=0, c \mathfrak{D}_{x}[\varphi]+\mathcal{M}_{\Omega}[\varphi]+a(x) \varphi+\lambda \varphi \geq 0\right\} .
$$

and for $c<0$

$$
\tilde{\lambda}_{p}^{\prime}\left(c \mathfrak{D}_{x}+\mathcal{M}_{\Omega}+\mathbf{a}\right)=\inf \left\{\lambda \mid \exists \varphi \in C^{1}(\Omega) \cap C(\bar{\Omega}), \varphi>0, \varphi\left(r_{1}\right)=0, c \mathfrak{D}_{x}[\varphi]+\mathcal{M}_{\Omega}[\varphi]+a(x) \varphi+\lambda \varphi \geq 0\right\} .
$$


In addition, there exists a positive function $\varphi_{1} \in C^{1}(\Omega) \cap C(\bar{\Omega})$ such that

$$
\left\{\begin{aligned}
c \mathfrak{D}_{x} \varphi_{1}+\mathcal{M}_{\Omega}\left[\varphi_{1}\right]+a \varphi_{1}+\lambda_{p} \varphi_{1} & =0 \text { in } \Omega=\left(r_{1}, r_{2}\right), \\
\varphi_{1} & >0 \text { in } \Omega=\left(r_{1}, r_{2}\right), \\
\varphi_{1}\left(r_{2}\right) & =0 \text { if } c>0, \\
\varphi_{1}\left(r_{1}\right) & =0 \text { if } c<0 .
\end{aligned}\right.
$$

The second property is a continuity result of the principal eigenvalue with respect to the domain and existence of a principal eigenfunction. Namely, we have

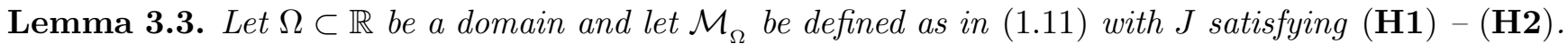
Assume further that $J$ is compactly supported and $a \in C(\mathbb{R}) \cap L^{\infty}(\mathbb{R})$. Let $\left(\Omega_{n}\right)_{n \in \mathbb{R}}$ be an increasing sequence of bounded domain of $\mathbb{R}$ such that $\lim _{n \rightarrow \infty} \Omega_{n}=\Omega, \Omega_{n} \subset \Omega_{n+1}$. Then, we have

$$
\lim _{n \rightarrow \infty} \lambda_{p}\left(c \mathfrak{D}_{x}+\mathcal{M}_{\Omega_{n}}+\mathbf{a}\right)=\lambda_{p}\left(c \mathfrak{D}_{x}+\mathcal{M}_{\Omega}+\mathbf{a}\right) .
$$

In addition, there exists a positive smooth $\varphi_{p}$ associated with $\lambda_{p}$.

As above for the Proposition 3.1, the proof of these results are already contained or follow from straightforward adaptation of the arguments developped in [4, 24] and [30] and as such we will omit their proofs.

3.1.2. Behaviour of $\lambda_{p}$ with respect to the speed. In this part we study more precisely the behaviour of the principal eigenvalue with respect to the speed $c$. Let us first show some useful equalities.

Proposition 3.4. Let $\Omega \subset \mathbb{R}$ be a domain, $a \in C(\bar{\Omega}) \cap L^{\infty}(\Omega)$ and assume that $J$ compactly supported

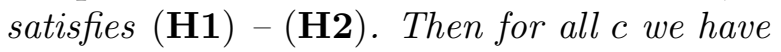

$$
\lambda_{p}\left(c \mathfrak{D}_{x}+\mathcal{M}_{\Omega}+\mathbf{a}(\mathbf{x})\right)=\lambda_{p}\left(-c \mathfrak{D}_{x}+\mathcal{M}_{\Omega}^{*}+\mathbf{a}(\mathbf{x})\right) .
$$

If in addition $\Omega$ is symmetric, in the sense that $\{-x \mid, x \in \Omega\}=\Omega$ then for all $c$ we have

$$
\lambda_{p}\left(c \mathfrak{D}_{x}+\mathcal{M}_{\Omega}+\mathbf{a}(\mathbf{x})\right)=\lambda_{p}\left(-c \mathfrak{D}_{x}+\mathcal{M}_{\Omega}^{*}+\mathbf{a}(-\mathbf{x})\right),
$$

Proof. Without any loss of generality we may assume that $c>0$. First, let us assume that $\Omega$ is bounded set, that is $\Omega:=\left(r_{1}, r_{2}\right)$. By Theorem $\left[3.2\right.$, there exists $\varphi, \varphi^{*} \in C(\bar{\Omega}) \cap C^{1}(\Omega)$ positive eigenfunctions associated respectively with $\lambda_{p}\left(c \mathfrak{D}_{x}+\mathcal{M}_{\Omega}+\mathbf{a}\right)$ and $\lambda_{p}\left(-c \mathfrak{D}_{x}+\mathcal{M}_{\Omega}^{*}+\mathbf{a}\right)$. Moreover they satisfies , $\varphi^{*}\left(r_{1}\right)=\varphi\left(r_{2}\right)=0$ and

$$
\begin{gathered}
c \mathfrak{D}_{x} \varphi+\mathcal{M}_{\Omega}[\varphi]+a \varphi+\lambda_{p}\left(c \mathfrak{D}_{x}+\mathcal{M}_{\Omega}+\mathbf{a}\right) \varphi=0 \\
-c \mathfrak{D}_{x} \varphi^{*}+\mathcal{M}_{\Omega}^{*}\left[\varphi^{*}\right]+a \varphi^{*}+\lambda_{p}\left(-c \mathfrak{D}_{x}+\mathcal{M}_{\Omega}^{*}+\mathbf{a}\right) \varphi^{*}=0
\end{gathered}
$$

Let us multiply by $\varphi^{*}$ the equation satisfied by $\varphi$ and integrate the resulting equation over $\Omega$. Integrating by parts and using the equation satisfied by $\varphi^{*}$ since $\varphi^{*}\left(r_{1}\right)=\varphi\left(r_{2}\right)=0$ it follows that

$$
\left(\lambda_{p}\left(c \mathfrak{D}_{x}+\mathcal{M}_{\Omega}+\mathbf{a}\right)-\lambda_{p}\left(-c \mathfrak{D}_{x}+\mathcal{M}_{\Omega}^{*}+\mathbf{a}\right)\right) \int_{\Omega} \varphi \varphi^{*}=0
$$

Thus the equality $\lambda_{p}\left(c \mathfrak{D}_{x}+\mathcal{M}_{\Omega}+\mathbf{a}\right)=\lambda_{p}\left(-c \mathfrak{D}_{x}+\mathcal{M}_{\Omega}^{*}+\mathbf{a}\right)$ holds true since $\varphi \varphi^{*}>0$. By continuity of $\lambda_{p}\left(c \mathfrak{D}_{x}+\mathcal{M}_{\Omega}+\mathbf{a}\right)$ with respect to the domain (Lemma 3.3) the equality (3.4) then holds true for any domain $\Omega$. The second equality is obtained just by observing that if $(\varphi, \lambda)$ satisfies

$$
c \mathfrak{D}_{x} \varphi+\mathcal{M}_{\Omega}[\varphi]+a \varphi+\lambda_{p}\left(c \mathfrak{D}_{x}+\mathcal{M}_{\Omega}+\mathbf{a}\right) \varphi=0
$$

then since $\Omega$ is symmetric the function $\psi(x):=\varphi(-x)$ satisfies

$$
-c \mathfrak{D}_{x} \psi+\mathcal{M}_{\Omega}^{*}[\psi]+a(-x) \psi+\lambda_{p}\left(c \mathfrak{D}_{x}+\mathcal{M}_{\Omega}+\mathbf{a}\right) \psi=0 .
$$

The equality (3.5) then follows by using the definition of the principal eigenvalue.

Next, we prove the following elementary property. 


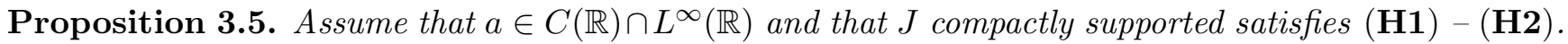
Assume further that $\sup _{\mathbb{R}} a(x)>0$. Then there exists $c_{0}^{+}>0$ such that for all $c>c_{0}^{+}$,

$$
\lambda_{p}\left(c \mathfrak{D}_{x}+\mathcal{M}+\mathbf{a}\right)>0 .
$$

Similarly there exists $c_{0}^{-}>0$ such that for all $c \leq-c_{0}^{-}$,

$$
\lambda_{p}\left(-c \mathfrak{D}_{x}+\mathcal{M}^{*}+\mathbf{a}\right)>0 .
$$

Proof. We treat the two situation $c>0$ and $c<0$ separately.

Case $c>0$ : In this situation, take $\varphi(x):=e^{-\lambda x}$, then we have

$$
c \mathfrak{D}_{x}[\varphi]+\mathcal{M}[\varphi]+a(x) \varphi=e^{-\lambda x}\left(-c \lambda+\int_{\mathbb{R}} J(z) e^{\lambda z} d z-1+a(x)\right) .
$$

Define

$$
c_{0}^{+}:=\inf _{\lambda>0} \frac{1}{\lambda}\left(\int_{\mathbb{R}} J(z) e^{\lambda z} d z-1+\sup _{x \in \mathbb{R}} a(x)\right) .
$$

$c_{0}^{+}$is bounded quantity since by an elementary computation we get

$$
\frac{1}{\lambda}\left(\int_{\mathbb{R}} J(z) e^{\lambda z} d z-1+\sup _{x \in \mathbb{R}} a(x)\right) \geq \int_{\mathbb{R}} J(z) z d z .
$$

By definition of $c_{0}^{+}$, for all $c>c_{0}^{+}$, there exists $\lambda(c)$ such that

$$
h(\lambda(c)):=-c \lambda(c)+\int_{\mathbb{R}} J(z) e^{\lambda(c) z} d z-1+\sup _{x \in \mathbb{R}} a(x)<0 .
$$

Whence for such $\lambda(c)$ we have

$$
\begin{aligned}
c \mathfrak{D}_{x}[\varphi]+\mathcal{M}[\varphi]+a(x) \varphi & =\varphi\left(-c \lambda+\int_{\mathbb{R}} J(z) e^{\lambda z} d z-1+\sup _{x \in \mathbb{R}} a(x)\right)+\left(a(x)-\sup _{x \in \mathbb{R}} a(x)\right) e^{-\lambda(c) x} \\
& \leq h(\lambda(c)) \varphi<0 .
\end{aligned}
$$

By definition of the principal eigenvalue of the operator $c \mathfrak{D}_{x}+\mathcal{M}+\mathbf{a},(\varphi,-h(\lambda(c))$ is then an admissible test function and therefore $\lambda_{p}\left(c \mathfrak{D}_{x}+\mathcal{M}+\mathbf{a}\right) \geq-h(\lambda(c))>0$.

Case $c<0$ : In this situation, again take $\varphi(x):=e^{-\lambda x}$ and observe that

$$
-c \mathfrak{D}_{x}[\varphi]+\mathcal{M}^{*}[\varphi]+a(x) \varphi=\varphi\left(c \lambda+\int_{\mathbb{R}} J(-z) e^{\lambda z} d z-1+a(x)\right) .
$$

Define

$$
c_{0}^{-}:=\inf _{\lambda>0} \frac{1}{\lambda}\left(\int_{\mathbb{R}} J(-z) e^{\lambda z} d z-1+\sup _{x \in \mathbb{R}} a(x)\right) .
$$

Again $c_{0}^{-}$is a bounded quantity, since a similar elementary computation shows that

$$
\frac{1}{\lambda}\left(\int_{\mathbb{R}} J(-z) e^{\lambda z} d z-1+\sup _{x \in \mathbb{R}} a(x)\right) \geq \int_{\mathbb{R}} J(-z) z d z .
$$

As above we can construct a admissible test function for $c<-c_{0}^{-}$. Indeed, when $c<-c_{0}^{-}$, then $-c>c_{0}^{-}$ and we can find $\lambda(c)>0$ such that

$$
\delta(c):=\left(c \lambda(c)+\int_{\mathbb{R}} J(-z) e^{\lambda(c) z} d z-1+\sup _{x \in \mathbb{R}} a(x)\right)<0 .
$$

Therefore $(\varphi, \delta(c))$ satisfies

$$
-c \mathfrak{D}_{x}[\varphi]+\mathcal{M}^{*}[\varphi]+a(x) \varphi-\delta(c) \varphi=\left(a(x)-\sup _{x \in \mathbb{R}} a(x)\right) \varphi \leq 0 .
$$

The couple $(\varphi,-\delta(c))$ is therefore a admissible test function for the principal eigenvalue of the operator $-c \mathfrak{D}_{x}+\mathcal{M}^{*}+\mathbf{a}(\mathbf{x})$ and as such we have $\lambda_{p}\left(-c \mathfrak{D}_{x}+\mathcal{M}^{*}+\mathbf{a}\right) \geq-\delta(c)>0$. By using Proposition 3.4, we then conclude

$$
\lambda_{p}\left(c \mathfrak{D}_{x}+\mathcal{M}+\mathbf{a}\right)=\lambda_{p}\left(-c \mathfrak{D}_{x}+\mathcal{M}^{*}+\mathbf{a}\right) \geq-\delta(c)>0 .
$$


Remark 2. Note that when $J$ is symmetric then $c_{0}^{+}=c_{0}^{-}=: c_{0}$ and we have the following quantitative bound for $c_{0}$ :

$$
c_{0} \geq \inf _{\lambda>0}\left(\lambda \int_{\mathbb{R}} J(z) z^{2} d z+\sup _{x \in \mathbb{R}} a(x) \frac{1}{\lambda}\right)=2 \sqrt{\int_{\mathbb{R}} J(z) z^{2} d z \cdot \sup _{x \in \mathbb{R}} a(x)} .
$$

Let us now show that for $c$ close to 0 then $\lambda_{p}\left(c \mathfrak{D}_{x}+\mathcal{M}+\mathbf{a}\right)<\mathbf{0}$. To do so let us first show it for $c>0$, namely

Lemma 3.6. Assume that $a \in C^{0, \alpha}(\mathbb{R}) \cap L^{\infty}(\mathbb{R})$ and $J$ with compact support satisfies $(\overline{\mathbf{H 1}})-(\overline{\mathbf{H 2}})$. Assume further that $\lambda_{p}(\mathcal{M}+\mathbf{a})<0$ then there exists $c^{*,+}>0$ such that for all $0<c<c^{*,+}, \lambda_{p}\left(c \mathfrak{D}_{x}+\mathcal{M}+\mathbf{a}\right)<0$.

Proof. To simplify the proof let us denote $\lambda_{0}:=\lambda_{p}(\mathcal{M}+\mathbf{a})<0$ and without any loss of generality let us assume that $\lambda_{p}(\mathcal{M}+\mathbf{a})$ is associated with a positive principal eigenfunction $\varphi_{p}$ otherwise using the Lipschitz continuity of $\lambda_{p}(\mathcal{M}+\mathbf{a})$ with respect to $a$, we can perturb $a$ by $a_{\varepsilon}$ with $a_{\varepsilon}$ such that $\lambda_{p}\left(\mathcal{M}+\mathbf{a}_{\varepsilon}\right)<\frac{\lambda_{0}}{2}$ and there exists $\varphi_{p}$ associated with $\lambda_{p}\left(\mathcal{M}+\mathbf{a}_{\varepsilon}\right)$.

Now thanks to the continuity of $\lambda_{p}\left(\mathcal{M}_{\Omega}+\mathbf{a}\right)$ with respect to the domain (Lemma 3.3 ), we have for some $R_{0}, \lambda_{p}\left(\mathcal{M}_{R_{0}}+\mathbf{a}\right)<\frac{\lambda_{0}}{2}$ and there exists $\varphi_{p}$ associated with $\lambda_{p}\left(\mathcal{M}_{R_{0}}+\mathbf{a}\right)$. Let us now introduce $\mathcal{M}_{\gamma, R_{0}}$ the following operator defined for $\varphi \in C\left(\left[-R_{0}, R_{0}\right]\right)$ by

$$
\mathcal{M}_{\gamma, R_{0}}[\varphi](x):=(1-\gamma) \int_{-R_{0}}^{R_{0}} J(x-y) \varphi(y) d y-\varphi(x) .
$$

Thanks to the continuity of $\lambda_{p}$ with respect to $a(x)$, for $\gamma>0$ small says, $\gamma \leq \gamma_{0}$ we have

$$
\lambda_{p}\left(\mathcal{M}_{\gamma, R_{0}}+\mathbf{a}\right) \leq \frac{\lambda_{0}}{4} .
$$

Next, we claim

Claim 3.7. For any $\delta>0$, there exists $\psi \in C^{1}\left(\left(-R_{0}, R_{0}\right)\right) \cap C_{c}\left(\left[-R_{0}, R_{0}\right]\right), \psi \geq 0$ such that

$$
\begin{aligned}
& \mathcal{M}_{\gamma, R_{0}}[\psi](x)+\left(a(x)+\lambda_{p}\left(\mathcal{M}_{\gamma, R_{0}}+\mathbf{a}\right)+2 \delta\right) \psi(x) \geq 0 \quad \text { for all } \quad x \in\left(-R_{0}, R_{0}\right) \\
& \inf _{x \in\left(-R_{0}, R_{0}\right)} \int_{-R_{0}}^{R_{0}} J(x-y) \psi(y) d y>0
\end{aligned}
$$

Assume for the moment that the claim holds true, then we can infer that

$$
\limsup _{c \rightarrow 0, c>0} \lambda_{p}\left(c \mathfrak{D}_{x}+\mathcal{M}_{R_{0}}+\mathbf{a}\right) \leq \lambda_{p}\left(\mathcal{M}_{\gamma, R_{0}}+\mathbf{a}\right)+2 \delta .
$$

Indeed, from the claim a direct computation shows that

$$
c \mathfrak{D}_{x}[\psi]+\mathcal{M}_{R_{0}}[\psi]+\left(a(x)+\lambda_{p}\left(\mathcal{M}_{\gamma, R_{0}}+\mathbf{a}\right)+2 \delta\right) \psi(x) \geq c \mathfrak{D}_{x}[\psi]+\gamma d_{0}
$$

where $d_{0}:=\inf _{x \in\left(-R_{0}, R_{0}\right)} \int_{-R_{0}}^{R_{0}} J(x-y) \psi(y) d y>0$. Therefore, for $c>0$ small enough we achieve

$$
c \mathfrak{D}_{x}[\psi]+\mathcal{M}_{R_{0}}[\psi]+\left(a(x)+\lambda_{p}\left(\mathcal{M}_{\gamma, R_{0}}+\mathbf{a}\right)+2 \delta\right) \psi(x) \geq c \mathfrak{D}_{x}[\psi]+\gamma d_{0}>0 .
$$

As a consequence, for $c>0$ small we then have

$$
\tilde{\lambda}_{p}^{\prime}\left(c \mathfrak{D}_{x}+\mathcal{M}_{R_{0}}+\mathbf{a}\right) \leq \lambda_{p}\left(\mathcal{M}_{\gamma, R_{0}}+a\right)+2 \delta .
$$

Now thanks to the Collatz Wieland type characterisation of $\lambda_{p}\left(c \mathfrak{D}_{x}+\mathcal{M}_{R_{0}}+\mathbf{a}\right)$, Theorem 3.2 , from the above inequality we deduce that for $c>0$ small

$$
\lambda_{p}\left(c \mathfrak{D}_{x}+\mathcal{M}_{R_{0}}+\mathbf{a}\right) \leq \lambda_{p}\left(\mathcal{M}_{\gamma, R_{0}}+\mathbf{a}\right)+2 \delta
$$

which enforces (3.6). Now by choosing $\delta$ small enough, we then get

$$
\limsup _{c \rightarrow 0, c>0} \lambda_{p}\left(c \mathfrak{D}_{x}+\mathcal{M}_{R_{0}}+\mathbf{a}\right)<\frac{\lambda_{0}}{8} .
$$


As a consequence there exists $c^{*,+}>0$, such that for all $0<c \leq c^{*,+} \lambda_{p}\left(c \mathfrak{D}_{x}+\mathcal{M}_{R_{0}}+\mathbf{a}\right) \leq \frac{\lambda_{0}}{16}$ and thanks to the monotone behaviour of $\lambda_{p}\left(c \mathfrak{D}_{x}+\mathcal{M}_{R_{0}}+\mathbf{a}\right)$ with respect to the domain, we have

$$
\lambda_{p}\left(c \mathfrak{D}_{x}+\mathcal{M}+\mathbf{a}\right) \leq \lambda_{p}\left(c \mathfrak{D}_{x}+\mathcal{M}_{R_{0}}+\mathbf{a}\right) \leq \frac{\lambda_{0}}{16}<0 .
$$

In order to conclude the proof let us now establish the claim.

Proof of the Claim. Let $\delta>0$ be a fixed. Arguing as in Claim 3.2 of [4], we can infer that there exists $\varepsilon_{0}>0$ such that for any $0<\varepsilon \leq \varepsilon_{0}$ then there exists $\varphi_{\varepsilon} \in C_{c}\left(\left(-R_{0}, R_{0}\right)\right)$ verifying

$$
\begin{aligned}
& \mathcal{M}_{\gamma, R_{0}}\left[\varphi_{\varepsilon}\right](x)+\left(a(x)+\lambda_{p}\left(\mathcal{M}_{\gamma, R_{0}}+\mathbf{a}\right)+\delta\right) \varphi_{\varepsilon}(x) \geq 0 \quad \text { for } \quad x \in\left(-R_{0}, R_{0}\right) . \\
& \left(-R_{0}+\varepsilon, R_{0}-\varepsilon\right) \subset \operatorname{supp}\left(\varphi_{\varepsilon}\right) .
\end{aligned}
$$

Since $J$ satisfies $(H 1-H 2)$ we can fix now $\varepsilon$ small, such that

$$
\inf _{x \in \Omega} \int_{-R_{0}}^{R_{0}} J(x-y) \varphi_{\varepsilon}(y) d y>0 .
$$

Observe that by construction, since $\varphi_{\varepsilon} \in C_{c}\left(\left(-R_{0}, R_{0}\right)\right)$, we can easily check that

$$
\mathcal{M}_{\gamma, \mathbb{R}}\left[\varphi_{\varepsilon}\right](x)+\left(a(x)+\lambda_{p}\left(\mathcal{M}_{\gamma, R_{0}}+\mathbf{a}\right)+\delta\right) \varphi_{\varepsilon}(x) \geq 0 \quad \text { for all } \quad x \in \mathbb{R} .
$$

Now, let $\zeta$ be a smooth mollifier of unit mass and with support in the unit interval and consider $\zeta_{\tau}:=\frac{1}{\tau} \zeta\left(\frac{z}{\tau}\right)$ for $\tau>0$

By taking $\psi:=\zeta_{\tau} \star \varphi_{\varepsilon}$ and observing that $\mathcal{M}_{\gamma, \mathbb{R}}[\psi](x)=\zeta_{\tau} \star\left(\mathcal{M}_{\gamma, \mathbb{R}}\left[\varphi_{\varepsilon}\right]\right)(x)$ for any $x \in \mathbb{R}$, we deduce that

$$
\begin{array}{lll}
\zeta_{\tau} \star\left(\mathcal{M}_{\gamma, \mathbb{R}}\left[\varphi_{\varepsilon}\right]+\left(a(x)+\lambda_{p}\left(\mathcal{M}_{\gamma, R_{0}}+\mathbf{a}\right)+\delta\right) \varphi_{\varepsilon}\right) \geq 0 & \text { for all } & x \in \mathbb{R}, \\
\mathcal{M}_{\gamma, \mathbb{R}}[\psi](x)+\left(\lambda_{p}\left(\mathcal{M}_{\gamma, R_{0}}+\mathbf{a}\right)+\delta\right) \psi(x)+\zeta_{\tau} \star\left(a(x) \varphi_{\varepsilon}\right)(x) \geq 0 & \text { for all } \quad & x \in \mathbb{R} .
\end{array}
$$

By adding and subtracting $a$, we then have for all $x \in \mathbb{R}$

$$
\mathcal{M}_{\gamma, \mathbb{R}}[\psi](x)+\left(a(x)+\lambda_{p}\left(\mathcal{M}_{\gamma, R_{0}}+\mathbf{a}\right)+\delta\right) \psi(x)+\int_{\mathbb{R}} \zeta_{\tau}(x-y) \varphi_{\varepsilon}(y)(a(y)-a(x)) d y \geq 0 .
$$

For $\tau$ small enough, say $\tau \leq \tau_{0}$, the function $\psi \in C_{c}^{\infty}\left(\left(-R_{0}, R_{0}\right)\right)$ and for all $x \in\left(-R_{0}, R_{0}\right)$ we have

$$
\begin{aligned}
\mathcal{M}_{\gamma, \mathbb{R}}[\psi](x) & =(1-\gamma) \int_{\mathbb{R}} J(x-y) \psi(y) d y-\psi(x), \\
& =(1-\gamma) \int_{-R_{0}}^{R_{0}} J(x-y) \psi(y) d y-\psi(x)=\mathcal{M}_{\gamma, R_{0}}[\psi](x) .
\end{aligned}
$$

Thus, from the above inequalities, for $\tau \leq \tau_{0}$, we get for all $x \in\left(-R_{0}, R_{0}\right)$,

$$
\mathcal{M}_{\gamma, R_{0}}[\psi](x)+\left(a(x)+\lambda_{p}\left(\mathcal{M}_{\gamma, R_{0}}+\mathbf{a}\right)+\delta\right) \psi(x)+\int_{\mathbb{R}} \zeta_{\tau}(x-y) \varphi_{\varepsilon}(y)(a(y)-a(x)) d y \geq 0 .
$$

Since $a$ is Hölder continuous, we can estimate the integral by

$$
\begin{aligned}
\left|\int_{\mathbb{R}} \zeta_{\tau}(x-y) \varphi_{\varepsilon}(y)(a(y)-a(x)) d y\right| & \leq \int_{\mathbb{R}} \zeta_{\tau}(x-y) \varphi_{\varepsilon}(y)\left|\frac{a(y)-a(x)}{|y-x|^{\alpha}}\right||x-y|^{\alpha} d y, \\
& \leq \kappa \tau^{\alpha} \psi(x),
\end{aligned}
$$

where $\kappa$ is the Hölder semi-norm of $a$. Thus, for $\tau$ small, says $\tau \leq \inf \left\{\left(\frac{\delta}{2 \kappa}\right)^{1 / \alpha}, \tau_{0}\right\}$, we have

$$
\mathcal{M}_{\gamma, R_{0}}[\psi](x)+\left(a(x)+\lambda_{p}\left(\mathcal{M}_{\gamma, R_{0}}+\mathbf{a}\right)+2 \delta\right) \psi(x) \geq 0 \quad \text { for all } \quad x \in\left(-R_{0}, R_{0}\right),
$$

In addition, since $\operatorname{supp}\left(\varphi_{\varepsilon}\right) \subset \operatorname{supp}(\psi)$, we then infer that

$$
\inf _{x \in\left(-R_{0}, R_{0}\right)} \int_{-R_{0}}^{R_{0}} J(x-y) \psi(y) d y>0,
$$


which end the proof of the claim.

Last let us prove that $\lambda_{p}\left(c \mathfrak{D}_{x}+\mathcal{M}+\mathbf{a}\right)<\mathbf{0}$ for $c<0$ and $c$ close to 0 . Namely,

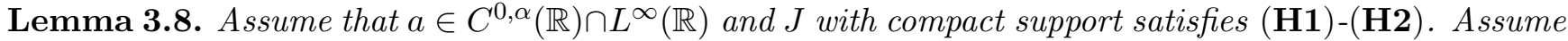
further that $\lambda_{p}(\mathcal{M}+\mathbf{a})<0$ then there exists $c^{*--}>0$ such that for all $-c^{*,-}<c<0, \lambda_{p}\left(c \mathfrak{D}_{x}+\mathcal{M}+\mathbf{a}\right)<0$.

Proof. To obtain $c^{*,-}$, let us observe that thanks to Proposition 3.4 we have

$$
\begin{aligned}
& \lambda_{p}\left(c \mathfrak{D}_{x}+\mathcal{M}+\mathbf{a}\right)=\lambda_{p}\left(-c \mathfrak{D}_{x}+\mathcal{M}^{*}+\mathbf{a}\right), \\
& \lambda_{p}(\mathcal{M}+\mathbf{a})=\lambda_{p}\left(\mathcal{M}^{*}+\mathbf{a}\right) .
\end{aligned}
$$

Let us then consider the operator $-c \mathfrak{D}_{x}+\mathcal{M}^{*}+\mathbf{a}$. Since $\lambda_{p}\left(\mathcal{M}^{*}+\mathbf{a}\right)<0$ and $-c>0$ and we can therefore apply the Lemma 3.6 to $-c \mathfrak{D}_{x}+\mathcal{M}^{*}+\mathbf{a}$. As a consequence there exists $\bar{c}$ such that for all $-c \leq \bar{c}$ we have $\lambda_{p}\left(-c \mathfrak{D}_{x}+\mathcal{M}^{*}+\mathbf{a}\right)<0$. Hence, by denoting $c^{*--}:=\bar{c}$ and by using the above equality, for all $c>-\bar{c}$, we have

$$
\lambda_{p}\left(c \mathfrak{D}_{x}+\mathcal{M}+\mathbf{a}\right)=\lambda_{p}\left(-c \mathfrak{D}_{x}+\mathcal{M}^{*}+\mathbf{a}\right)<0 .
$$

By Propositions 3.5 and 3.4, Lemma 3.6 and Lemma 3.8 we can then define

$$
\begin{aligned}
& c^{*,+}:=\sup \left\{c>0 \mid \forall 0<c^{\prime} \leq c, \lambda_{p}\left(c^{\prime} \mathfrak{D}_{x}+\mathcal{M}+\mathbf{a}\right)<0\right\}, \\
& c^{*,-}:=\inf \left\{c<0 \mid \forall c \leq c^{\prime}<0, \lambda_{p}\left(c^{\prime} \mathfrak{D}_{x}+\mathcal{M}+\mathbf{a}\right)<0\right\},
\end{aligned}
$$

and

$$
\begin{aligned}
c^{* *++} & :=\inf \left\{c>0 \mid \forall c^{\prime} \geq c, \lambda_{p}\left(c^{\prime} \mathfrak{D}_{x}+\mathcal{M}+\mathbf{a}\right) \geq 0\right\}, \\
c^{* *-} & :=\sup \left\{c<0 \mid \forall c^{\prime} \leq c, \lambda_{p}\left(c^{\prime} \mathfrak{D}_{x}+\mathcal{M}+\mathbf{a}\right) \geq 0\right\} .
\end{aligned}
$$

Observe that from the definition of $c^{*, \pm}, c^{* *, \pm}$ and thanks to Proposition 3.4, the Theorem 1.3 will then be proved as soon as the optimal persistence criteria (Theorem 1.1) is proved.

3.2. Principal eigenvalue for nonlocal operators with an elliptic part. In this section we recall the definition and behaviour of the principal eigenvalue for general integrodifferential operator $\mathfrak{L}$ of the form

$$
\mathfrak{L}[\varphi]:=\mathcal{E}[\varphi]+\mathcal{L}_{\Omega}[\varphi]
$$

where $\mathcal{E}$ is an elliptic operator of the form

$$
\mathcal{E}[\varphi]:=\alpha(x) \mathfrak{D}_{x x}[\varphi]+\beta(x) \mathfrak{D}_{x}[\varphi]+\gamma(x) \varphi
$$

where $\alpha(x)>0$. As in $[11,15,4,31,30,45$, we can check that the quantity

$$
\lambda_{p}(\mathfrak{L}):=\sup \left\{\lambda \in \mathbb{R} \mid \exists \varphi \in C(\bar{\Omega}) \cap W_{l o c}^{2,1}(\Omega), \varphi>0, \mathfrak{L}[\varphi]+\lambda \varphi \leq 0\right\}
$$

is well defined and satisfies all the properties defined in Proposition 3.1. Moreover we also have the following Collatz-Wieland characterisation

Theorem 3.9 ([31]). Assume that $\Omega=\left(r_{1}, r_{2}\right) \subset \mathbb{R}$ (with $\left.r_{1}<r_{2}\right)$ is a bounded domain and let $\alpha, \beta, \gamma \in$ $C^{0, \alpha}(\Omega) \cap C(\bar{\Omega})$ and $J$ with compact support satisfying $(\overline{\mathbf{H 1}})-(\overline{\mathbf{H 2}})$. Assume further that $\alpha>0$, then $\lambda_{p}(\mathfrak{L})$ is the principal eigenvalue of $\mathfrak{L}$, meaning that there exists a positive smooth function $\varphi_{p}$ associated to $\lambda_{p}(\mathfrak{L})$, such that

$$
\begin{aligned}
& \mathfrak{L}\left[\varphi_{p}\right](x)+\lambda_{p} \varphi_{p}(x)=0 \quad \text { for all } \quad x \in \Omega, \\
& \varphi_{p}\left(r_{1}\right)=\varphi_{p}\left(r_{2}\right)=0 .
\end{aligned}
$$

Moreover, $\lambda_{p}(\mathfrak{L})$ satisfies the following Collatz-Wieland characterisation:

$$
\lambda_{p}(\mathfrak{L})=\lambda_{p}^{\prime}(\mathfrak{L}):=\inf \left\{\lambda \in \mathbb{R} \mid \exists \varphi \in C^{2}(\Omega) \cap C(\bar{\Omega}), \varphi>0, \varphi\left(r_{1}\right)=\varphi\left(r_{2}\right)=0, \mathfrak{L}[\varphi]+\lambda \varphi \geq 0\right\} .
$$




\section{Analysis of the Regularised PROBlem}

In this section we construct a positive solution $u_{\varepsilon}$ of a regularised version of (1.5). For $\varepsilon>0$ let us introduce the following regularised problem :

$$
\varepsilon \mathfrak{D}_{x x}[u](x)+c \mathfrak{D}_{x}[u](x)+\mathcal{M}[u](x)+f(x, u(x))=0 \quad \text { for all } \quad x \in \mathbb{R} .
$$

Then, for a fixed $c>0$ such that $\lambda_{p}\left(c \mathfrak{D}_{x}+\mathcal{M}+\mathbf{a}\right)<0$, we will show that we can find $\varepsilon_{0}(c)$ such that for $\varepsilon \leq \varepsilon_{0}$, the problem (4.1) admits a unique positive non trivial solution. Namely, we prove the following

Theorem 4.1. Assume that $f$ and $J$ satisfy $(\overline{\mathbf{H 1}})-(\overline{\mathbf{H} 4})$ and that $c>0$ is such that $\lambda_{p}\left(c \mathfrak{D}_{x}+\mathcal{M}+\right.$ $\left.\partial_{s} f(x, 0)\right)<0$. Then there exists $\varepsilon_{0}>0$ such that for all $0<\varepsilon \leq \varepsilon_{0}$ there exists a unique positive continuous function, $u_{\varepsilon}$, solution of (4.1).

The next subsections deal with the proof of Theorem 4.1. The proof of the Theorem 4.1 uses standard approximation schemes that we can find for example in [23, 9, 10]. To simplify the presentation we break down the proof in three parts, the next two subsection being devoted to each one of them. In the first part, subsection 4.1, we introduce the following approximated problem :

$$
\begin{aligned}
& \varepsilon \mathfrak{D}_{x x}[u](x)+c \mathfrak{D}_{x}[u](x)+\mathcal{M}_{R}[u](x)+f(x, u(x))=0 \quad \text { for all } \quad x \in(-R, R) \\
& u(-R)=u(R)=0
\end{aligned}
$$

and we find $\varepsilon_{0}$ and $R_{0}$ positive constants such that for all $R \geq R_{0}$ and $\varepsilon \leq \varepsilon_{0}$ the problem (4.2)-(4.3) set on a $(-R, R)$ has a unique solution. More precisely, we prove

Theorem 4.2. Assume that $f$ and $J$ satisfy $(\overline{\mathbf{H 1}})-(\underline{\mathbf{H} 4})$ and that $c>0$ is such that $\lambda_{p}\left(c \mathfrak{D}_{x}+\mathcal{M}+\right.$ $\left.\partial_{s} f(x, 0)\right)<0$. Then there exists $R_{0}>0$ and $\varepsilon_{0}>0$ such that for all $0<\varepsilon \leq \varepsilon_{0}, R \geq R_{0}$ there exists a unique positive continuous function, $u_{\varepsilon, R}$, solution to (4.2)-(4.3). Moreover, for any smooth initial data $v_{0} \in C^{1}((-R, R)), v_{0} \geq \not \equiv$ then the solution $v(t, x)$ of the following Cauchy problems

$$
\begin{aligned}
& \partial_{t} v(t, x)=\varepsilon \mathfrak{D}_{x x}[v](t, x)+c \mathfrak{D}_{x}[v](t, x)+\mathcal{M}_{R}[v](t, x)+f(x, v(t, x)) \quad \text { for } \quad t>0, x \in(-R, R) \\
& v(t,-R)=v(t, R)=0 \quad \text { for } \quad t>0 \\
& v(0, x)=v_{0}(x) \quad \text { for } \quad x \in(-R, R),
\end{aligned}
$$

converges uniformly to $u_{\varepsilon, R}$.

Then in a second part,subsection 4.2, by using a standard limiting procedure and a-priori estimates, we show that $\varepsilon_{0}$ is independent of $R$ and that for any $0<\varepsilon \leq \varepsilon_{0}$ a positive non trivial solution to (4.1) can be constructed from the sequences $\left(u_{\varepsilon, R_{n}}\right)_{n \in \mathbb{N}}$ with $R_{n} \rightarrow \infty$. In the last part, subsection 4.3 we prove the uniqueness of the solution of (4.1).

4.1. Existence of a unique non-trivial positive solution in a bounded domain. We start this subsection by proving the uniqueness of the positive solution of (4.2)-(4.3). Our proof follows a standard argument that we can find for example in [7, 28]. Let $u$ and $v$ be two non negative solutions to (4.2) $-(4.3)$. Then thanks to the maximum principle and using the equation satisfied by $u$ and $v$ there exists $M>0$ such that $M>u>0, M>v>0$ and moreover we have

$$
\begin{array}{r}
\mathfrak{D}_{x}[u](-R)>0, \mathfrak{D}_{x}[v](-R)>0 \\
\mathfrak{D}_{x}[u](R)<0, \mathfrak{D}_{x}[v](R)<0 .
\end{array}
$$

So there exists $\sigma_{0}>0$ such that $\frac{1}{\sigma_{0}} v \leq u \leq \sigma_{0} v$. From this inequalities we can define

$$
\sigma^{*}:=\inf \{\tau>0 \mid \sigma v\}
$$

and by definition of $\sigma^{*}$, we have $u \leq \sigma^{*} v$. We claim that

Claim 4.3. $\sigma^{*} \leq 1$.

Note that by proving the claim we deduce that $u \leq v$ and since the role of $u$ and $v$ can be interchanged we then obtain $v \leq u$ as well, which proves the uniqueness. 
Proof of the Claim: Assume by contradiction that $\sigma^{*}>1$. Then by using that $v$ is a solution, we can check that $\sigma^{*} v$ is a super-solution to (4.2)-(4.3). Now since $u \leq \sigma^{*} v$ and $f$ is locally Lipschitz thanks to the strong maximum principle, we can check that either $u<\sigma^{*} v$ or $u \equiv \sigma^{*} v$. In the latter case, since $\frac{f(x, s)}{s}$ is strictly decreasing we then have the following contradiction

$$
\begin{aligned}
0=\varepsilon \mathfrak{D}_{x x}[u]+c \mathfrak{D}_{x}[u]+\mathcal{M}_{R}[u]+\frac{f(x, u)}{u} u & =\varepsilon \mathfrak{D}_{x x}\left[\sigma^{*} v\right]+c \mathfrak{D}_{x}\left[\sigma^{*} v\right]+\mathcal{M}_{R}\left[\sigma^{*} v\right]+\frac{f\left(x, \sigma^{*} v\right)}{\sigma^{*} v} \sigma^{*} v \\
& <\sigma^{*}\left(\varepsilon \mathfrak{D}_{x x}[v]+c \mathfrak{D}_{x}[v]+\mathcal{M}_{R}[v]+f(x, v)\right)=0 .
\end{aligned}
$$

In the other case, we also get a contradiction. Namely, since $u<\sigma^{*} v$ and thanks to the equations satisfied by $u$ and $\sigma^{*} v$ we can see that $\mathfrak{D}_{x}[u](-R)<\sigma^{*} \mathfrak{D}_{x}[v](-R)$ and $\mathfrak{D}_{x}[u](R)>\sigma^{*} \mathfrak{D}_{x}[v](R)$. Therefore $u \leq\left(\sigma^{*}-\varepsilon\right) v$ for some $\varepsilon$ small contradicting the definition of $\sigma^{*}$.

Let us prove now that for $\varepsilon$ small and $R$ well chosen, a positive solution to (4.2) $-(4.3)$ exists. For convienience, we use the following notation $a(x):=\partial_{s} f(x, 0)$. Note that the problem (4.2) -(4.3) can be solved using standard sub and super-solution scheme (see 23) and that large constants are super-solutions of this problem. So, in order to construct a solution it is enough to construct a bounded sub-solution of (4.2) - (4.3) and thanks to the nature of the problem (4.2)-(4.3), it is enough to show that

$$
\lambda_{p}\left(\varepsilon \mathfrak{D}_{x x}+c \mathfrak{D}_{x}+\mathcal{M}_{R}+\mathbf{a}\right)<0 .
$$

Indeed, let assume that $\lambda_{p}\left(\varepsilon \mathfrak{D}_{x x}+c \mathfrak{D}_{x}+\mathcal{M}_{R}+\mathbf{a}\right)<0$ and let $\varphi_{p}$ the associated positive eigenfunction. Then a straightforward computation shows that for small $\kappa$ the function $\kappa \varphi_{p}$ satisfies $\kappa \varphi_{p}(R)=$ $\kappa \varphi_{p}(-R)=0$ and

$$
\varepsilon \mathfrak{D}_{x x}\left[\kappa \varphi_{p}\right](x)+c \mathfrak{D}_{x}\left[\kappa \varphi_{p}\right](x)+\mathcal{M}_{R}\left[\kappa \varphi_{p}\right](x)+f\left(x, \kappa \varphi_{p}(x)\right)=-\lambda_{p} \kappa \varphi_{p}(x)+o\left(\kappa \varphi_{p}(x)\right) \geq 0 .
$$

As a consequence, for small $\kappa$ the function $\kappa \varphi_{p}$ is a subsolution of (4.2)-(4.3).

Let us now prove that (4.4) holds true for small $\varepsilon$ and $R$ well chosen. For that we prove the following

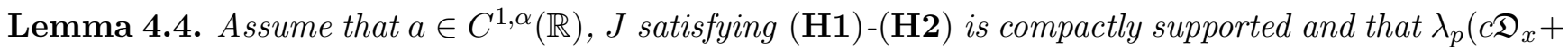
$\mathcal{M}+\mathbf{a})<0$. Then there exists $R_{0}$ such that

$$
\limsup _{\varepsilon \rightarrow 0, \varepsilon>0} \lambda_{p}\left(\varepsilon \mathfrak{D}_{x x}+c \mathfrak{D}_{x}+\mathcal{M}_{R_{0}}+\mathbf{a}\right)<0 .
$$

Assume for the moment that this Lemma holds true. Then there exists $\varepsilon_{0}\left(c, R_{0}\right)$ such that for all $\varepsilon \leq \varepsilon_{0}$ we have

$$
\lambda_{p}\left(\varepsilon \mathfrak{D}_{x x}+c \mathfrak{D}_{x}+\mathcal{M}_{R_{0}}+\mathbf{a}\right)<0 .
$$

By using that $\lambda_{p}\left(\varepsilon \mathfrak{D}_{x x}+c \mathfrak{D}_{x}+\mathcal{M}_{R}+\mathbf{a}\right)$ is monotone non increasing with respect to $R$ we then conclude that for all $R \geq R_{0}$ and all $\varepsilon \leq \varepsilon_{0}$ we have

$$
\lambda_{p}\left(\varepsilon \mathfrak{D}_{x x}+c \mathfrak{D}_{x}+\mathcal{M}_{R}+\mathbf{a}\right) \leq \lambda_{p}\left(\varepsilon \mathfrak{D}_{x x}+c \mathfrak{D}_{x}+\mathcal{M}_{R_{0}}+\mathbf{a}\right)<0 .
$$

Now, thanks to the Collatz-Wieland type characterisation of $\lambda_{p}\left(\varepsilon \mathfrak{D}_{x x}+c \mathfrak{D}_{x}+\mathcal{M}_{R}+\mathbf{a}\right)$ (Theorem 3.9 ), to prove this Lemma it is enough to show that there exists $R_{0}>0$ such that we have $\lambda_{p}\left(c \mathfrak{D}_{x}+\mathcal{M}_{R_{0}}+\mathbf{a}\right)<0$ and for small $\delta>0$, say $\delta \leq-\frac{\lambda_{p}\left(c \mathfrak{D}_{x}+\mathcal{M}_{R_{0}}+\mathbf{a}\right)}{2}$, we can find $\psi>0, \psi \in W^{2,1}(\Omega) \cap L^{\infty}(\Omega)$ and $\varepsilon(\delta)$ such that for all $\varepsilon \leq \varepsilon_{\delta}$ :

$$
\left\{\begin{array}{l}
\varepsilon \mathfrak{D}_{x x}[\psi](x)+c \mathfrak{D}_{x}[\psi](x)+\mathcal{M}_{R_{0}}[\psi](x)+a(x) \psi(x)+\left(\lambda_{p}\left(c \mathfrak{D}_{x}+\mathcal{M}_{R_{0}}+\mathbf{a}\right)+\delta\right) \psi(x) \geq 0, \text { on }\left(-R_{0}, R_{0}\right) \\
\psi\left(-R_{0}\right)=\psi\left(R_{0}\right)=0 .
\end{array}\right.
$$

To simplify the presentation of the proof of this Lemma we decompose it into two steps. 
Step One : Some a priori estimates. Let $R>0$ and let $\varphi_{p, c, R}$ be the positive eigenfunction associated with $\lambda\left(c \mathfrak{D}_{x}+\mathcal{M}_{R}+\mathbf{a}\right)$. Without any loss of generality, we can assume that $\varphi_{p, c, R} \leq 1$. Let $d_{1}(c, R)$ be the following positive constant:

$$
d_{1}:=\inf _{x \in(-R, R)} \int_{-R}^{R} J(x-y) \varphi_{p, c, R}(y) d y
$$

From the equation satisfied by $\varphi_{p, c, R}$ we can check that for all $x \in(-R, R)$ we have

$$
c \mathfrak{D}_{x}\left[\varphi_{p, c, R}\right](x)+\left(\lambda_{p}\left(c \mathfrak{D}_{x}+\mathcal{M}_{R}+\mathbf{a}\right)-1+a(x)\right) \varphi_{p, c, R}(x)=-\int_{-R}^{R} J(x-y) \varphi_{p, c, R}(y) d y \leq-d_{1} .
$$

Therefore, since for all $c$ and $R$ we have $\lambda_{p}\left(c \mathfrak{D}_{x}+\mathcal{M}_{R}+\mathbf{a}\right) \geq-\sup _{x \in(-R, R)} a(x)$, we deduce that

$$
\mathfrak{D}_{x}\left[\varphi_{p, c}\right](x) \leq-\frac{d_{1}}{c}+\frac{\gamma_{0}}{c} \varphi_{p, c, R}(x)
$$

with $\gamma_{0}:=\sup _{x \in \mathbb{R}} a(x)-\inf _{x \in \mathbb{R}} a(x)+1$.

Now we claim that

Claim 4.5. For all $\delta>0$, there exists $c(\delta)>c$, such that for all $c<c^{\prime} \leq c(\delta)$ we have

$$
c \mathfrak{D}_{x}\left[\varphi_{p, c^{\prime}, R}\right](x)+\mathcal{M}_{R}\left[\varphi_{p, c^{\prime}, R}\right](x)+\left(a(x)+\lambda_{p}\left(c^{\prime} \mathfrak{D}_{x}+\mathcal{M}_{R}+\mathbf{a}\right)+\delta\right) \varphi_{p, c^{\prime}, R}(x) \geq \frac{c^{\prime}-c}{c^{\prime}} d_{1}\left(c^{\prime}\right)+\frac{\delta}{2} \varphi_{p, c^{\prime}, R}(x) .
$$

Proof. Let $\delta>0$ be fixed and for $c^{\prime}>c$ and $\varphi_{p, c^{\prime}}$, let us compute

$$
c \mathfrak{D}_{x}\left[\varphi_{p, c^{\prime}, R}\right](x)+\mathcal{M}_{R}\left[\varphi_{p, c^{\prime}, R}\right](x)+\left(a(x)+\lambda_{p}\left(c^{\prime} \mathfrak{D}_{x}+\mathcal{M}_{R}+\mathbf{a}\right)+\delta\right) \varphi_{p, c^{\prime}, R}(x) .
$$

By (4.5) we can infer that for any $c^{\prime}>c$

$$
\begin{aligned}
c \mathfrak{D}_{x}\left[\varphi_{p, c^{\prime}, R}\right]+\mathcal{M}_{R}\left[\varphi_{p, c^{\prime}, R}\right]+\left(a(x)+\lambda_{p}\left(c^{\prime} \mathfrak{D}_{x}+\mathcal{M}_{R}+\mathbf{a}\right)+\delta\right) \varphi_{p, c^{\prime}, R} & =\left(c-c^{\prime}\right) \mathfrak{D}_{x}\left[\varphi_{p, c^{\prime}, R}\right]+\delta \varphi_{p, c^{\prime}, R}, \\
& \geq \frac{c^{\prime}-c}{c^{\prime}} d_{1}\left(c^{\prime}\right)+\left(\delta-\frac{\left(c^{\prime}-c\right)}{c^{\prime}} \gamma_{0}\right) \varphi_{p, c^{\prime}, R} .
\end{aligned}
$$

Thus, the claim holds true by taking $c(\delta) \leq c\left(1+\frac{\delta}{2 \gamma_{0}}\right)$.

Remark 3. Note that $c(\delta)$ does not depend of $R$.

Step two : Construction of a good test function for $\varepsilon$ small.

First, let us remark that since $J$ and $f$ satisfies $(\underline{\mathbf{H 1}})-(\underline{\mathbf{H 4}})$ and $\lambda_{p}\left(c \mathfrak{D}_{x}+\mathcal{M}+\mathbf{a}\right)<0$ by using Lemma 3.3 we have

$$
\lim _{R \rightarrow+\infty} \lambda_{p}\left(c \mathfrak{D}_{x}+\mathcal{M}_{R}+\mathbf{a}\right)=\lambda_{p}\left(c \mathfrak{D}_{x}+\mathcal{M}+\mathbf{a}\right)<0
$$

and so there exists $R_{0}>0$ such that

$$
\lambda_{p}\left(c \mathfrak{D}_{x}+\mathcal{M}_{R_{0}}+\mathbf{a}\right)<\frac{\lambda_{p}\left(c \mathfrak{D}_{x}+\mathcal{M}+\mathbf{a}\right)}{2}<0 .
$$

Let us fix $\delta \leq-\frac{\lambda_{p}\left(c \mathfrak{D}_{x}+\mathcal{M}_{R_{0}}+\mathbf{a}\right)}{2}$ and let us start our construction of $\psi$.

First, thanks to the continuity of $\lambda_{p}\left(c \mathfrak{D}_{x}+\mathcal{M}_{R_{0}}+\mathbf{a}\right)$ with respect to the speed $c$, there exists $c_{0}>c$ such that for all $c<c^{\prime} \leq c_{0}$, we have

$$
\lambda_{p}\left(c^{\prime} \mathfrak{D}_{x}+\mathcal{M}_{R_{0}}+\mathbf{a}\right) \leq \lambda_{p}\left(c \mathfrak{D}_{x}+\mathcal{M}_{R_{0}}+\mathbf{a}\right)+\frac{\delta}{4} .
$$

From our choice of $c_{0}$ let us observe that for all $c_{0} \geq c^{\prime}>c$ we have

$$
\lambda_{p}\left(c^{\prime} \mathfrak{D}_{x}+\mathcal{M}_{R_{0}}+\mathbf{a}\right) \leq \frac{7}{8} \lambda_{p}\left(c \mathfrak{D}_{x}+\mathcal{M}_{R_{0}}+\mathbf{a}\right)<\frac{7}{16} \lambda_{p}\left(c \mathfrak{D}_{x}+\mathcal{M}+\mathbf{a}\right)<0 .
$$


Now thanks to the Claim 4.5 there exists $c(\delta)$ such that for all $c<c^{\prime} \leq c(\delta)$ the principal eigenfunction $\varphi_{p, c^{\prime}, R_{0}}$ verifies

$$
c \mathfrak{D}_{x}\left[\varphi_{p, c^{\prime}, R_{0}}\right]+\mathcal{M}_{R_{0}}\left[\varphi_{p, c^{\prime}, R_{0}}\right]+\left(a(x)+\lambda_{p}\left(c^{\prime} \mathfrak{D}_{x}+\mathcal{M}_{R_{0}}+\mathbf{a}\right)+\delta\right) \varphi_{p, c^{\prime}, R_{0}} \geq \frac{c^{\prime}-c}{c^{\prime}} d_{1}\left(c^{\prime}\right)+\frac{\delta}{2} \varphi_{p, c^{\prime}, R_{0}} .
$$

Fix now $c^{\prime} \leq \inf \left\{c_{0}, c(\delta)\right\}$ then from the above inequality and by using (4.8) we achieve

$$
c \mathfrak{D}_{x}\left[\varphi_{p, c^{\prime}, R_{0}}\right]+\mathcal{M}_{R_{0}}\left[\varphi_{p, c^{\prime}, R_{0}}\right]+\left(a(x)+\lambda_{p}\left(c \mathfrak{D}_{x}+\mathcal{M}_{R_{0}}+\mathbf{a}\right)+\delta\right) \varphi_{p, c^{\prime}, R_{0}} \geq \frac{c^{\prime}-c}{c^{\prime}} d_{1}\left(c^{\prime}\right)+\frac{\delta}{4} \varphi_{p, c^{\prime}, R_{0}} .
$$

For $\tau<\frac{1}{4}$, let us now choose a family of increasing cut-off function $\zeta_{\tau}(x)$ such that

$$
\begin{cases}\zeta_{\tau}(x)=0 & \text { for } \quad-R_{0} \leq x \leq-R_{0}+\tau \\ \zeta_{\tau}^{\prime}(x) \geq 0 & \text { for } \quad-R_{0}+\tau<x<-R_{0}+\sqrt{\tau} \\ \zeta_{\tau}(x)=1 & \text { for } \quad-R_{0}+\sqrt{\tau} \leq x \leq R_{0}\end{cases}
$$

and that for some uniform constant $C_{0}$ verifies $\left|\zeta_{\tau}^{\prime}(x)\right| \leq \frac{C_{0}}{\sqrt{\tau}}$. Choose $\tau_{0}<\frac{1}{4}$ small such that

$$
\inf _{x \in \Omega} \int_{-R_{0}+\sqrt{\tau_{0}}}^{R_{0}} J(x-y) \varphi_{p, c^{\prime}, R_{0}}(y) d y:=d_{0}>0 .
$$

Let us define $\psi:=\varphi_{p, c^{\prime}, R_{0}}(x) \zeta_{\tau}(x)$ and for $\tau \leq \tau_{0}$ let us compute

$$
c \mathfrak{D}_{x}[\psi]+\mathcal{M}_{R_{0}}[\psi]+\left(a(x)+\lambda_{p}\left(c \mathfrak{D}_{x}+\mathcal{M}_{R_{0}}+\mathbf{a}\right)+\delta\right) \psi .
$$

Observe that $\psi \equiv 0$ for $x \leq-R_{0}+\tau$ and $\tau \leq \tau_{0}$, so we have for $x \leq-R_{0}+\tau$

$$
\begin{aligned}
c \mathfrak{D}_{x}[\psi]+\mathcal{M}_{R_{0}}[\psi]+\left(a(x)+\lambda_{p}\left(c \mathfrak{D}_{x}+\mathcal{M}_{R_{0}}+\mathbf{a}\right)+\delta\right) \psi & =\int_{-R_{0}}^{R_{0}} J(x-y) \psi(y) d y \\
& \geq \int_{-R_{0}+\sqrt{\tau_{0}}}^{R_{0}} J(x-y) \varphi_{p, c^{\prime}, R_{0}}(y) d y \geq d_{0} .
\end{aligned}
$$

On the other hand, for $x \in\left(-R_{0}+\sqrt{\tau}, R_{0}\right)$ we have $\zeta_{\tau}(x)=1$ and thanks to (4.9) we deduce that

$$
\begin{aligned}
c \mathfrak{D}_{x}[\psi]+\mathcal{M}_{R_{0}}[\psi]+\left(a(x)+\lambda_{p}\left(c \mathfrak{D}_{x}+\mathcal{M}_{R_{0}}+\mathbf{a}\right)+\delta\right) \psi & \geq \int_{-R_{0}}^{R_{0}} J(x-y) \varphi_{p, c^{\prime}, R_{0}}(y)\left(\zeta_{\tau}(y)-1\right) d y+\frac{c^{\prime}-c}{c^{\prime}} d_{1}+\frac{\delta}{4} \psi . \\
& \geq \int_{-R_{0}}^{-R_{0}+\sqrt{\tau}} J(x-y) \varphi_{p, c^{\prime}, R_{0}}(y) d y+\frac{c^{\prime}-c}{c^{\prime}} d_{1}+\frac{\delta}{4} \varphi_{p, c^{\prime}, R_{0}} . \\
& \geq-\|J\|_{\infty} \sqrt{\tau}+\frac{c^{\prime}-c}{c^{\prime}} d_{1}+\frac{\delta}{4} \varphi_{p, c^{\prime}, R_{0}} .
\end{aligned}
$$

By taking $\tau \leq \inf \left\{\left(\frac{c^{\prime}-c}{2 c^{\prime}\|J\|_{\infty}} d_{1}\right)^{2}, \tau_{0}\right\}$ we then achieve for $x \in\left(-R_{0}+\sqrt{\tau}, R_{0}\right)$

$$
c \mathfrak{D}_{x}[\psi]+\mathcal{M}_{R_{0}}[\psi]+\left(a(x)+\lambda_{p}\left(c \mathfrak{D}_{x}+\mathcal{M}_{R_{0}}+\mathbf{a}\right)+\delta\right) \psi \geq \frac{c^{\prime}-c}{2 c^{\prime}} d_{1}+\frac{\delta}{4} \varphi_{p, c^{\prime}, R_{0}} \geq 0 .
$$

Similarly, thanks to (4.9) and since $\zeta_{\tau}$ is a monotone non decreasing function, a short computation shows that on $\left(-R_{0}+\tau,-R_{0}+\sqrt{\tau}\right)$

$$
\begin{array}{r}
c \mathfrak{D}_{x}[\psi]+\mathcal{M}_{R_{0}}[\psi]+\left(a(x)+\lambda_{p}\left(c \mathfrak{D}_{x}+\mathcal{M}_{R_{0}}+a\right)+\delta\right) \psi \geq \int_{-R_{0}}^{R_{0}} J(x-y) \varphi_{p, c^{\prime}, R_{0}}(y)\left(\zeta_{\tau}(y)-\zeta_{\tau}(x)\right) d y \\
+\zeta_{\tau}(x)\left(\frac{c^{\prime}-c}{c^{\prime}} d_{1}+\frac{\delta}{4} \varphi_{p, c^{\prime}, R_{0}}\right) .
\end{array}
$$


Define $I:=\int_{-R_{0}}^{R_{0}} J(x-y) \varphi_{p, c^{\prime}, R_{0}}(y)\left(\zeta_{\tau}(y)-\zeta_{\tau}(x)\right) d y$, and let us estimate $I$.

Since $\zeta_{\tau} \equiv 1$ in $\left(-R_{0}+\sqrt{\tau}, R_{0}\right), \zeta_{\tau}$ is monotone non decreasing and $\left|\zeta_{\tau}^{\prime}(x)\right| \leq \frac{C_{0}}{\sqrt{\tau}}$ and since $\tau \leq \tau_{0}$ and $x \in\left(-R_{0}+\tau,-R_{0}+\sqrt{\tau}\right)$ we can estimate $I$ as follows:

$$
\begin{aligned}
I & \geq\left(1-\zeta_{\tau}(x)\right) \int_{-R_{0}+\sqrt{\tau}}^{R_{0}} J(x-y) \varphi_{p, c^{\prime}}(y) d y+\int_{-R_{0}}^{x} J(x-y) \varphi_{p, c^{\prime}}(y)\left(\zeta_{\tau}(y)-\zeta_{\tau}(x)\right) d y \\
& \geq\left(1-\zeta_{\tau}(x)\right) d_{0}-\int_{-R_{0}}^{x} J(x-y)\left|\frac{\zeta_{\tau}(y)-\zeta_{\tau}(x)}{y-x}\right||x-y| d y \\
& \geq\left(1-\zeta_{\tau}(x)\right) d_{0}-\frac{C_{0}}{\sqrt{\tau}} \int_{0}^{\sqrt{\tau}} J(z)|z| d z \\
& \geq\left(1-\zeta_{\tau}(x)\right) d_{0}-\frac{C_{0} \sqrt{\tau}\|J\|_{\infty}}{2} .
\end{aligned}
$$

As a consequence, by setting $d^{*}:=\inf \left\{d_{0}, \frac{c^{\prime}-c}{2 c^{\prime}} d_{1}\right\}$ and by choosing $\tau$ small, say

$$
\tau \leq \tau_{1}:=\inf \left\{\tau_{0},\left(\frac{c^{\prime}-c}{2 c^{\prime}\|J\|_{\infty}} d_{1}\right)^{2},\left(\frac{d^{*}}{C_{0}\|J\|_{\infty}}\right)^{2}\right\}
$$

we then achieve for all $x \in\left(-R_{0}+\tau,-R_{0}+\sqrt{\tau}\right)$

$$
c \mathfrak{D}_{x}[\psi]+\mathcal{M}_{R_{0}}[\psi]+\left(a(x)+\lambda_{p}\left(c \mathfrak{D}_{x}+\mathcal{M}_{R_{0}}+\mathbf{a}\right)+\delta\right) \psi \geq \zeta_{\tau}(x) \frac{\delta}{4} \varphi_{p, c^{\prime}, R_{0}}(x)+\frac{d^{*}}{2} .
$$

By using that $a \in C^{1, \alpha}(\mathbb{R})$ we can check that the eigenfunction $\varphi_{p, c^{\prime}, R_{0}} \in C^{2}\left(\left(-R_{0}, R_{0}\right)\right)$ and by definition of $\psi$ this implies that $\psi \in C^{2}\left(\left(-R_{0}, R_{0}\right)\right)$. Moreover collecting (4.10), (4.11) and (4.12), for any $x \in\left(-R_{0}, R_{0}\right)$, we also have

$$
\begin{aligned}
& \varepsilon \mathfrak{D}_{x x}[\psi]+c \mathfrak{D}_{x}[\psi]+\mathcal{M}_{R_{0}}[\psi]+\left(a(x)+\lambda_{p}\left(c \mathfrak{D}_{x}+\mathcal{M}_{R_{0}}+\mathbf{a}\right)+\delta\right) \psi \geq \varepsilon \mathfrak{D}_{x x}[\psi]+\frac{d^{*}}{2} \\
& \psi\left(-R_{0}\right)=\psi\left(R_{0}\right)=0
\end{aligned}
$$

which for $\varepsilon$ small enough says $\varepsilon \leq \varepsilon^{*}:=\frac{d^{*}}{4\|\psi\|_{C^{2}}}$ satisfies

$$
\varepsilon \mathfrak{D}_{x x}[\psi]+c \mathfrak{D}_{x}[\psi]+\mathcal{M}_{R_{0}}[\psi]+\left(a(x)+\lambda_{p}\left(c \mathfrak{D}_{x}+\mathcal{M}_{R_{0}}+\mathbf{a}\right)+\delta\right) \psi \geq \frac{d^{*}}{4}>0,
$$

ending thus the proof of the Lemma.

Remark 4. From the above construction, since $\psi \equiv 0$ in $\left(-R_{0},-R_{0}+\tau\right)$, we can easily observe that for all $\varepsilon \leq \varepsilon^{*}$ the function $\psi$ is still a good test function in $\left(r_{1}, R_{0}\right)$ for all $r_{1} \leq-R_{0}$.

Remark 5. Observe that for small $\kappa$, the function $\kappa \psi$ can also serve as a sub-solution of the problem (4.2)-(4.3) defined in $\left(-R_{0}, R_{0}\right)$. Indeed, a straightforward computation shows that

$$
\begin{aligned}
\varepsilon \mathfrak{D}_{x x}[\kappa \psi]+c \mathfrak{D}_{x} \kappa \psi+\mathcal{M}_{R_{0}}[\kappa \psi]+f(x, \kappa \psi) & \geq \frac{\kappa d^{*}}{4}+\left(-\lambda_{p}\left(c \mathfrak{D}_{x}+\mathcal{M}_{R_{0}}+\mathbf{a}\right)-\delta\right) \kappa \psi+o(\kappa \psi) \\
& \geq \frac{\kappa d^{*}}{4}-\frac{\lambda_{p}\left(c \mathfrak{D}_{x}+\mathcal{M}_{R_{0}}+\mathbf{a}\right)}{2} \kappa \psi+o(\kappa \psi) \geq 0 .
\end{aligned}
$$

Thus since large constants are super-solutions of the problem and the problem admits a unique positive solution we will have

$$
\kappa \psi \leq u_{\varepsilon, R_{0}}
$$


4.2. Existence of a non-trivial positive solution in $\mathbb{R}$. Thanks to the previous subsection, for a fixed $c>0$ we know that there exists $\varepsilon_{0}>0$ and $R_{0}>0$ such that for any $0<\varepsilon \leq \varepsilon_{0}$ and $R \geq R_{0}$ there exists $u_{\varepsilon, R}$ a unique positive solution to (4.2) - (4.3). In addition, for a fixed $\varepsilon$ and for any $R_{1}>R_{2} \geq R_{0}$, the solution $u_{\varepsilon, R_{1}}$ is a super-solution for the problem

$$
\begin{aligned}
& \varepsilon \mathfrak{D}_{x x}[u]+c \mathfrak{D}_{x}[u]+\mathcal{M}_{R_{2}}[u]+f(x, u)=0 \text { in }\left(-R_{2}, R_{2}\right) \\
& u\left(-R_{2}\right)=u\left(R_{2}\right)=0
\end{aligned}
$$

Therefore by a sweeping argument we get

$$
u_{\varepsilon, R_{2}} \leq u_{\varepsilon, R_{1}} \quad \text { in } \quad\left(-R_{2}, R_{2}\right) .
$$

Thus, for $\varepsilon$ fixed the map $R \mapsto u_{\varepsilon, R}$ is monotone increasing. As a consequence and thanks to Remark 5 for all $R \geq R_{0}$ we will always have

$$
\kappa \psi \leq u_{\varepsilon, R_{0}} \leq u_{\varepsilon, R} \quad \text { on } \quad\left(-R_{0}, R_{0}\right)
$$

for some $\kappa>0$ independent of $\varepsilon$.

On the other hand, since $f$ satisfies $(\underline{\mathbf{H 3}})$-( $(\underline{\mathbf{H} 4})$, we can find a constant $M$ such that for all $R \geq R_{0}$ this constant is a super-solution of the problem (4.2) - (4.3). Thus, by uniqueness of the solution, we then have $u_{\varepsilon, R} \leq M$ for all $\varepsilon \leq \varepsilon_{0}$ and $R \geq R_{0}$.

Let us fix now $\varepsilon \leq \varepsilon_{0}$ and let $\left(R_{n}\right)_{n \in \mathbb{N}}$ be an increasing sequence starting from $R_{0}$ and such that $R_{n} \rightarrow+\infty$. Let us denote by $\left(u_{n}\right)_{n \in \mathbb{N}}$ the corresponding sequence of solutions of (4.2)-(4.3) set on $\left(-R_{n}, R_{n}\right)$. Since $u_{n}$ is uniformly bounded, by using local elliptic estimate we can check that $\left(u_{n}\right)_{n \in \mathbb{N}}$ is bounded uniformly in $C^{2, \alpha}\left(-R_{n}, R_{n}\right)$ and therefore by a diagonal extraction process we can extract of the sequence $\left(u_{n}\right)_{n \in \mathbb{N}}$ a subsequence, still denoted $\left(u_{n}\right)_{n \in \mathbb{N}}$ that converges locally uniformly to a positive bounded function $u_{\varepsilon}$ which is solution to

$$
\varepsilon \mathfrak{D}_{x x}\left[u_{\varepsilon}\right]+c \mathfrak{D}_{x}\left[u_{\varepsilon}\right]+\mathcal{M}\left[u_{\varepsilon}\right]+f\left(x, u_{\varepsilon}\right)=0 \quad \text { in } \quad \mathbb{R} .
$$

Moreover, thanks to (4.13), we have $\kappa \psi \leq u_{\varepsilon} \leq M$ in $\left(-R_{0}, R_{0}\right)$. Note that the latter bound is independent of $\varepsilon$ so we get for all $\varepsilon \leq \varepsilon_{0}$

$$
\kappa \psi \mathbb{1}_{\left(-R_{0}, R_{0}\right)} \leq u_{\varepsilon} \leq M .
$$

Whence, for all $\varepsilon \leq \varepsilon_{0}$ there exists a non trivial solution $u_{\varepsilon}$ to (4.1) thus proving Theorem 4.1.

4.3. Uniqueness of the solution of (4.1). Let us now prove that the solution $u_{\varepsilon}$ is unique. We argue by contradiction and assume that there exists $v$ another positive solution to (4.1). We will show that $u_{\varepsilon}<v$ and $v \leq u_{\varepsilon}$ which will give us our contradiction.

Let us first remark that $v$ is a supersolution of problem (4.2) - (4.3) for any $R>0$. Therefore by a standard sweeping argument we conclude that $v \geq u_{\varepsilon, R}$ for all $R \geq R_{0}$. Since $u_{\varepsilon, R}$ is monotone with respect to $R$, it follows that $v \geq u_{\varepsilon}$. By assumption $v \not \equiv u_{\varepsilon}$ almost everywhere and by using the strong maximum principle we then have $v>u_{\varepsilon}$. Our main task is then to prove the inequality $v \leq u_{\varepsilon}$. To do so we will use a sweeping type argument. But first we will establish some useful properties of $v$ that will constantly used along our proof. Namely,

Proposition 4.6. Assume that $f$ and $J$ satisfy $(\overline{\mathbf{H 1}})-(\overline{\mathbf{H} 4})$ and assume further that $J$ satisfies $(\mathbf{H 6})$. Let $v \in C_{l o c}^{2}(\mathbb{R})$ be a positive bounded solution to (4.1). Then $v \in L^{1}(\mathbb{R})$.

Proof. By assumption since $f$ is in $C^{1}$ and $v \in L^{\infty}(\mathbb{R}) \cap C_{l o c}^{2}(\mathbb{R})$ is a solution to (4.1), by using interior elliptic regularity we deduce that $\mathfrak{D}_{x}[v] \in L^{\infty}(\mathbb{R})$. Now to prove that $v \in L^{1}$ and let us integrate (4.1) over $(-R, R)$, we then get

$\varepsilon\left(\mathfrak{D}_{x}[v](R)-\mathfrak{D}_{x}[v](-R)\right)+c(v(R)-v(-R))+\int_{-R}^{R} \int_{\mathbb{R}} J(-z)[v(x+z)-v(x)] d z d x=-\int_{-R}^{R} f(x, v(x)) d x$. 
Let us set $\mathcal{A}_{R}:=\varepsilon\left(\mathfrak{D}_{x}[v](R)-\mathfrak{D}_{x}[v](-R)\right)+c(v(R)-v(-R))$ and by using that $J$ has a first moment, that $v$ is smooth, and Fubini's Theorem we can rewrite the equality as follows

$$
\begin{aligned}
-\int_{-R}^{R} f(x, v(x)) d x & =\mathcal{A}_{R}+\int_{-R}^{R} \int_{\mathbb{R}} J(-z) z \int_{0}^{1} \mathfrak{D}_{x}[v](x+s z) d s d z d x \\
& =\mathcal{A}_{R}+\int_{0}^{1} \int_{\mathbb{R}} J(-z) z[v(R+s z)-v(-R+s z]) d s d z
\end{aligned}
$$

Therefore, since $v$ and $\mathfrak{D}_{x}[v]$ are bounded we get for all $R>0$

$$
-\int_{-R}^{R} f(x, v(x)) d x<C_{0}:=2\|v\|_{\infty}\left(c+\int_{\mathbb{R}} J(z)|z| d z\right)+2 \varepsilon\left\|\mathfrak{D}_{x}[v]\right\|_{\infty} .
$$

Now since $f$ satisfies $(\overline{\mathbf{H} 3})$ and $(\overline{\mathbf{H} 4})$ we can find $R_{0}>0$ and $\kappa_{0}>0$ such that $f(x, s)<-\kappa_{0} s$ for all $|x|>R_{0}$ and $s \in \mathbb{R}^{+}$. Whence, for all $R>R_{0}$ we get from (4.15) that

$$
\begin{gathered}
-\int_{-R}^{-R_{0}} f(x, v(x)) d x-\int_{R_{0}}^{R} f(x, v(x)) d x \leq\left|\int_{-R_{0}}^{R_{0}} f(x, v(x)) d x\right|+C_{0} \\
\kappa_{0}\left(\int_{-R}^{-R_{0}} v(x) d x+\int_{R_{0}}^{R} v(x) d x\right) \leq C_{0}+2 R_{0}\|f\|_{\infty},
\end{gathered}
$$

which by using that $v$ is bounded implies that

$$
\int_{-R}^{R} v(x) d x \leq \frac{C_{0}+2 R_{0}\left[\|f\|_{\infty}+\|v\|_{\infty}\right]}{\kappa_{0}}
$$

Since $v$ is positive, the later estimate shows that $v \in L^{1}$.

Remark 6. Note that as a corollary of the fact $v \in L^{1}$, since $v$ is smooth we must have $\lim _{x \rightarrow \pm \infty} v(x)=0$. In addition since $v \in L^{1}(\mathbb{R}) \cap L^{\infty}(\mathbb{R})$, by interpolation $v \in L^{p}$ for any $1 \leq p$. In particular, $v \in L^{2}(\mathbb{R})$.

Remark 7. We observe that the above proof only rely on elementary computations which will be true as well when $\varepsilon=0$. Therefore we then have that any bounded positive smooth solution to (1.5) is in $L^{1}(\mathbb{R})$.

Equipped with the Proposition 4.6, we are now in position to prove $v \leq u_{\varepsilon}$. To this end, we will use a sweeping type argument, which essentially relies on two main steps. First we prove that $v \leq \tau_{0} u_{\varepsilon}$ for some $\tau_{0}>0$ and then in a second step we show that $v \leq \tau u_{\varepsilon}$ for all $\tau \geq 1$. Let us start by proving the following:

Claim 4.7. There exists $\tau_{0}>0$ such that $v(x) \leq \tau_{0} u_{\varepsilon}(x)$ for all $x \in \mathbb{R}$

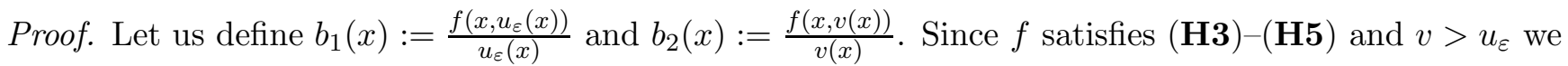
have $b_{1}(x)>b_{2}(x)$ and therefore $u_{\varepsilon}, v$ satisfy:

$$
\begin{aligned}
& \varepsilon \mathfrak{D}_{x x}\left[u_{\varepsilon}\right](x)+c \mathfrak{D}_{x}\left[u_{\varepsilon}\right](x)+\mathcal{M}\left[u_{\varepsilon}\right](x)+b_{2}(x) u_{\varepsilon}(x)<0 \\
& \varepsilon \mathfrak{D}_{x x}[v](x)+c \mathfrak{D}_{x}[v](x)+\mathcal{M}[v](x)+b_{2}(x) v(x)=0 .
\end{aligned}
$$

By using that $f$ satisfies ([for all $|x| \geq R_{0}$. Now since $u_{\varepsilon}$ and $v$ are bounded and positive the following positive quantity are well defined

$$
M_{0}:=\max _{x \in\left[-R_{0}, R_{0}\right]} v(x) \quad m_{0}:=\min _{x \in\left[R_{0}, R_{0}\right]} u_{\varepsilon}(x) .
$$

By considering now the function $C u_{\varepsilon}$ with $C:=\frac{M_{0}}{m_{0}}+1$ we then have $C u_{\varepsilon}>v$ in $\left[-R_{0}, R_{0}\right]$. Therefore since $0=\lim _{|x| \rightarrow+\infty} v(x) \leq \lim _{|x| \rightarrow+\infty} C u_{\varepsilon}(x)$, by using the weak comparison principle, Theorem 2.1, we then conclude that $v \leq C u_{\varepsilon}$. 
From the Claim 4.7 the following quantity is then well defined

$$
\tau^{*}:=\inf \left\{\tau>0, \mid v \leq \tau u_{\varepsilon}\right\}
$$

and we claim that

Claim 4.8. $\tau^{*} \leq 1$

Note that by proving the claim we end our proof since we then achieved that $v \leq \tau^{*} u \leq u$.

Proof. Again, to show this claim we will argue by contradiction and assume that $\tau^{*}>1$. By definition we have $v \leq \tau^{*} u_{\varepsilon}$ and by the strong comparison principle, Theorem 2.2, either $v<\tau^{*} u_{\varepsilon}$ or $v \equiv \tau^{*} u_{\varepsilon}$. In the later case, we get the contradiction

$$
\begin{aligned}
0=\varepsilon \mathfrak{D}_{x x}[v]+c \mathfrak{D}_{x}[v]+\mathcal{M}[v]+\frac{f(x, v)}{v} v & =\varepsilon \mathfrak{D}_{x x}\left[\sigma^{*} u_{\varepsilon}\right]+c \mathfrak{D}_{x}\left[\sigma^{*} u_{\varepsilon}\right]+\mathcal{M}\left[\sigma^{*} u_{\varepsilon}\right]+\frac{f\left(x, \sigma^{*} u_{\varepsilon}\right)}{\sigma^{*} u_{\varepsilon}} \sigma^{*} u_{\varepsilon} \\
& <\sigma^{*}\left(\varepsilon \mathfrak{D}_{x x}\left[u_{\varepsilon}\right]+c \mathfrak{D}_{x}\left[u_{\varepsilon}\right]+\mathcal{M}\left[u_{\varepsilon}\right]+f\left(x, u_{\varepsilon}\right)\right)=0 .
\end{aligned}
$$

Let us now obtain a contradiction in the other situation. Since $v<\tau^{*} u_{\varepsilon}$, we may find $\tau^{\prime}<\tau^{*}$ such that $v(x)<\tau^{\prime} u_{\varepsilon}(x)$ for all $x \in\left[-R_{0}, R_{0}\right]$. In addition we can check that $v$ and $\tau^{\prime} u_{\varepsilon}$ satisfy

$$
\begin{cases}\varepsilon \mathfrak{D}_{x x}\left[\tau^{\prime} u_{\varepsilon}\right](x)+c \mathfrak{D}_{x}\left[\tau^{\prime} u_{\varepsilon}\right](x)+\mathcal{M}\left[\tau^{\prime} u_{\varepsilon}\right](x)+b_{2}(x)\left(\tau^{\prime} u_{\varepsilon}(x)\right)<0 & \text { for all }|x|>R_{0} \\ \varepsilon \mathfrak{D}_{x x}[v](x)+c \mathfrak{D}_{x}[v](x)+\mathcal{M}[v](x)+b_{2}(x) v(x)=0 & \text { for all }|x|>R_{0}, \\ v(x)<\tau^{\prime} u_{\varepsilon}(x) & \text { for all } x \in\left[-R_{0}, R_{0}\right], \\ \lim _{|x| \rightarrow \infty} v(x) \leq \lim _{|x| \rightarrow+\infty} \tau^{\prime} u_{\varepsilon}(x) . & \end{cases}
$$

Thus by applying the weak comparison principle, Theorem 2.1, we get $v \leq \tau^{\prime} u_{\varepsilon}$ contradicting the definition of $\tau^{*}$. Whence we must have $\tau^{*} \leq 1$.

\section{The Sufficient COndition: Existence of A NON tRivial Steady State}

In this section, by analysing the singular limit problem (4.1) as $\varepsilon \rightarrow 0$ we construct a positive solution $\bar{u}$ of (1.5) and prove the sufficient condition stated in Theorem 1.1. The proof being similar for $c>0$ and $c<0$, we only present the construction for the case $c>0$ and when necessary we add some remarks to handle the case $c<0$.

So let us now analyse the singular limit of $u_{\varepsilon}$ when $\varepsilon \rightarrow 0$ and construct a positive non trivial solution to (1.5). From (4.14) we know that $M \geq u_{\varepsilon} \geq \kappa \psi \mathbb{1}_{\left(-R_{0}, R_{0}\right)}$ with $M$ and $\kappa$ constants independent of $\varepsilon$. Next, we show that there exists $C_{1}$ such that for all $\varepsilon$, we have

$$
\left|\mathfrak{D}_{x}\left[u_{\varepsilon}\right]\right| \leq C_{1}
$$

To obtain such estimate, we will first establish the following estimate:

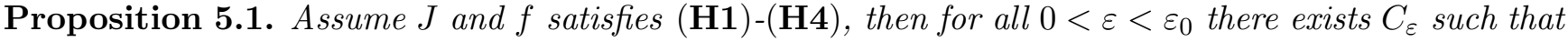

$$
\left\|u_{\varepsilon}\right\|_{H_{1}(\mathbb{R})} \leq C_{\varepsilon} .
$$

Assume for the moment that the proposition holds true and let us prove that it implies (5.1). Indeed, since $u_{\varepsilon}$ is bounded in $H_{1}(\mathbb{R})$ and smooth we can then infer that $\lim _{|x| \rightarrow+\infty} \mathfrak{D}_{x}\left[u_{\varepsilon}\right](x)=0$ and $\lim _{|x| \rightarrow+\infty} u_{\varepsilon}(x)=0$ and as a consequence, the quantities $\sup _{x \in \mathbb{R}} \mathfrak{D}_{x}\left[u_{\varepsilon}\right](x)$ and $\inf _{x \in \mathbb{R}} \mathfrak{D}_{x}\left[u_{\varepsilon}\right](x)$ are achieved at some points $x_{0}, x_{1}$ in $\mathbb{R}$. Now, by using (4.1) we deduce that at these points we have

$$
-M-\sup _{x \in \mathbb{R}, s \in[0, M]}|f|(x, s) \leq c \mathfrak{D}_{x}\left[u_{\varepsilon}\right]\left(x_{i}\right) \leq M+\sup _{x \in \mathbb{R}, s \in[0, M]}|f|(x, s)
$$

and thus

$$
\left|\mathfrak{D}_{x}\left[u_{\varepsilon}\right]\left(x_{i}\right)\right| \leq C_{1}:=\frac{M+\sup _{x \in \mathbb{R}, s \in[0, M]}|f|(x, s)}{c} .
$$

Now thanks to (5.1) we can construct a non trivial solution to (1.5). Indeed, take a sequence $\left(\varepsilon_{n}\right)_{n \in \mathbb{N}}$ such that $\varepsilon_{n} \rightarrow 0$ and let $u_{\varepsilon_{n}}$ be the non trivial solution of the regularised equation (4.1) with $\varepsilon_{n}$. Thanks to (15.1) and (4.14) the sequence $\left(u_{\varepsilon_{n}}\right)_{n \in \mathbb{N}}$ is uniformly bounded in $C^{1}(\mathbb{R})$ and therefore by a diagonal extraction process we can find a subsequence that converges locally uniformly and pointwise to a non 
negative function $\tilde{u}$. Moreover, $\kappa \psi \mathbb{1}_{\left(-R_{0}, R_{0}\right)} \leq \tilde{u} \leq M$ and passing to the limit in the equation in the sense of distribution, we can check that $\tilde{u}$ satisfies in a weak sense

$$
c \mathfrak{D}_{x}[\tilde{u}]+\mathcal{M}[\tilde{u}]+f(x, \tilde{u})=0 .
$$

Recall that $c \neq 0$, thereby by a standard regularity argument, we can see that $\tilde{u}$ is smooth and satisfies the above equation strongly whence

$$
c \mathfrak{D}_{x}[\tilde{u}](x)+\mathcal{M}[\tilde{u}](x)+f(x, \tilde{u}(x))=0 \quad \text { for all } \quad x \in \mathbb{R} .
$$

To finish our proof let us prove the proposition.

Proof of the Proposition. To prove that, let us first recall that since $\varepsilon>0$ and $u_{\varepsilon}$ is a bounded solution of (4.1) by using standard regularity estimates it follows that $\mathfrak{D}_{x}\left[u_{\varepsilon}\right]$ is also uniformly bounded in $\mathbb{R}$. So by multiplying (4.1) by $u_{\varepsilon}$ and by integrating the resulting equation over $(-R, R)$ we then obtain

$\varepsilon \int_{-R}^{R} u_{\varepsilon} \mathfrak{D}_{x x}\left[u_{\varepsilon}\right](x) d x+\frac{c}{2} \int_{-R}^{R} \mathfrak{D}_{x}\left[u_{\varepsilon}^{2}\right](x) d x+\int_{-R}^{R}\left(u_{\varepsilon}(x) \mathcal{M}\left[u_{\varepsilon}\right](x)-u_{\varepsilon}^{2}(x)\right) d x+\int_{-R}^{R} f\left(x, u_{\varepsilon}(x)\right) u_{\varepsilon}(x) d x=0$.

By integrating by part the first terms and rearranging the other terms it then follows that

$$
\varepsilon \int_{-R}^{R}\left|\mathfrak{D}_{x}\left[u_{\varepsilon}\right]\right|^{2} d x-\int_{-R}^{R}\left(u_{\varepsilon}(x) \mathcal{M}\left[u_{\varepsilon}\right](x)-u_{\varepsilon}^{2}(x)\right) d x=\int_{-R}^{R} f\left(x, u_{\varepsilon}(x)\right) u_{\varepsilon}(x) d x+I_{R}
$$

where $I_{R}$ is the quantity

$$
I_{R}:=\frac{c}{2}\left[u_{\varepsilon}^{2}(R)-u_{\varepsilon}^{2}(-R)\right]+u_{\varepsilon}(R) \mathfrak{D}_{x}\left[u_{\varepsilon}\right](R)-u_{\varepsilon}(-R) \mathfrak{D}_{x}\left[u_{\varepsilon}\right](-R) .
$$

Let us estimates all the terms in the equality (5.2). By Proposition 4.6 and Remark 6 it follows that $u_{\varepsilon} \in L^{2}$ and $u_{\varepsilon}( \pm R) \rightarrow 0$ as $R \rightarrow+\infty$, and thus $\lim _{R \rightarrow \infty} I_{R}=0$ since $\left|\mathfrak{D}_{x}\left[u_{\varepsilon}\right]\right|$ is bounded uniformly. As a consequence there exists a positive constant $C_{1}$ such that for all $R>0$

$$
I_{R} \leq C_{1} .
$$

Now by recalling that $f$ satisfies [H3 $\mathbf{H 4}$ and $0<u_{\varepsilon} \leq M:=\|S\|_{\infty}$ since by Proposition 4.6 ] $u_{\varepsilon}$ is integrable we also deduce that for some positive constant $C_{2}$

$$
\left|\int_{-R}^{R} f\left(x, u_{\varepsilon}\right) u_{\varepsilon}(x) d x\right| \leq \int_{-R}^{R}\left|f\left(x, u_{\varepsilon}(x)\right)\right| u_{\varepsilon}(x) d x \leq \sup _{x \in \mathbb{R}, s \in[0, M]}|f(x, s)| \int_{-R}^{R} u_{\varepsilon}(x) d x \leq C_{2} .
$$

Lastly, since $u_{\varepsilon}$ is bounded and integrable we also have the following estimate for some positive constant $C_{3}$ :

$$
\begin{aligned}
-\int_{-R}^{R}\left(u_{\varepsilon}(x) \mathcal{M}\left[u_{\varepsilon}\right](x)-u_{\varepsilon}^{2}(x)\right) d x & \geq \int_{-R}^{R} u_{\varepsilon}^{2}(x) d x-\int_{-R}^{R} \int_{\mathbb{R}} J(x-y) u_{\varepsilon}(x) u_{\varepsilon}(y) d x d y \\
& \geq \int_{-R}^{R} u_{\varepsilon}^{2}(x) d x-M\|J\|_{\infty} \int_{-R}^{R} u_{\varepsilon}(x) d x \\
& \geq \int_{-R}^{R} u_{\varepsilon}^{2}(x) d x-C_{3} .
\end{aligned}
$$

Combining (5.2) with (5.3), (5.4) and (5.5), we then achieve for all $R>0$

$$
\varepsilon \int_{-R}^{R}\left|\mathfrak{D}_{x}\left[u_{\varepsilon}\right]\right|^{2} d x+\int_{-R}^{R} u_{\varepsilon}^{2} d x \leq C_{1}+C_{2}+C_{3}<+\infty .
$$




\section{UNIQUENESS}

Having constructed a smooth positive solution of $(1.5)$ in $L^{1}(\mathbb{R})$, we continue our proof of Theorem 1.1 by proving its uniqueness. Unfortunately, the argument used to proved the uniqueness of $u_{\varepsilon}$ does not applies here and we require a new approach. To obtain the uniqueness of $u$, we argue by contradiction and assume that $v \in C^{1}(\mathbb{R}) \cap L^{\infty}(\mathbb{R})$ is another positive solution. Our argumentation is rather long and for convenience we decompose it three subsections

6.1. $v$ is $H^{1}$. Let us show that the solution $v$ is in $H^{1}$. Thanks to Proposition 4.6 and Remarks 7 and 6 , we already know that $v \in L^{2}$ and we only need to prove that $\mathfrak{D}_{x}[v] \in L^{2}(\mathbb{R})$.

To do so, let us multiply the equation satisfied by $v$ by $\mathfrak{D}_{x}[v]$ and integrate it over $(-R, R)$. By rearranging the term, we get

$$
\begin{aligned}
c \int_{-R}^{R}\left(\mathfrak{D}_{x}[v]\right)^{2} & =\int_{-R}^{R} v \mathfrak{D}_{x}[v]-\int_{-R}^{R} \int_{\mathbb{R}} J(x-y) \mathfrak{D}_{x}[v](x) v(y) d x d y-\int_{-R}^{R} \frac{f(x, v)}{v} v \mathfrak{D}_{x}[v] \\
& \leq \frac{1}{2}\left[v^{2}(R)-v^{2}(-R)\right]+\left\|\mathfrak{D}_{x}[v]\right\|_{\infty}\left(\int_{-R}^{R} \int_{\mathbb{R}} J(x-y) v(y) d x d y+\left\|f_{s}(x, 0)\right\|_{\infty}\|v\|_{L^{1}}\right) \\
& \leq\|v\|_{\infty}+\left\|\mathfrak{D}_{x}[v]\right\|_{\infty}\|v\|_{L^{1}}\left(1+\left\|f_{s}(x, 0)\right\|_{\infty}\right) .
\end{aligned}
$$

6.2. On the sign of some principal eigenvalue. Let us assume there exists $v \in L^{1}(\mathbb{R}) \cap C^{0,1}(\mathbb{R})$ such that $v>0$ and $v$ satisfies

$$
c \mathfrak{D}_{x}[v](x)+\mathcal{M}[v](x)+f(x, v(x)) \geq 0 \quad \text { for almost every } \quad x \in \mathbb{R} .
$$

Set $b(x):=\frac{f(x, v(x))}{v(x)}$ and let us consider the principal eigenvalue $\lambda_{p}\left(c \mathfrak{D}_{x}+\mathcal{M}+\mathbf{b}\right)$ of the operator $c \mathfrak{D}_{x}+\mathcal{M}+\mathbf{b}$ where $\lambda_{p}$ is defined in sub-section [3.1. Let us also define the following quantity

$\lambda_{p}^{\prime \prime}\left(-c \mathfrak{D}_{x}+\mathcal{M}^{*}+\mathbf{b}\right):=\inf \left\{\lambda \in \mathbb{R} \mid \exists \varphi>0, \varphi \in W^{1, \infty}(\mathbb{R}) \cap C^{1}(\mathbb{R}) \quad\right.$ s.t. $\left.\quad c \mathfrak{D}_{x}[\varphi]+\mathcal{M}[\varphi]+(b(x)+\lambda) \varphi \geq 0\right\}$.

We claim that

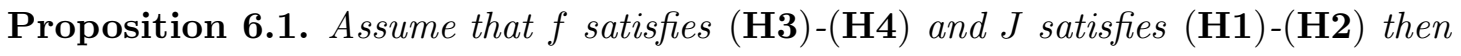

$$
\lambda_{p}^{\prime \prime}\left(-c \mathfrak{D}_{x}+\mathcal{M}^{*}+\mathbf{b}\right) \leq \lambda_{p}\left(c \mathfrak{D}_{x}+\mathcal{M}+\mathbf{b}\right) \leq 0 .
$$

Moreover, there exists $\varphi>0, \varphi \in W^{1,1}(\mathbb{R}) \cap W^{1, \infty}(\mathbb{R}) \cap C^{1}(\mathbb{R})$ such that

$$
-c \mathfrak{D}_{x}[\varphi]+\mathcal{M}^{*}[\varphi]+b(x) \varphi \geq 0 .
$$

Proof. We split our proof into two main steps, namely we start by showing that

$$
\lambda_{p}\left(c \mathfrak{D}_{x}+\mathcal{M}+\mathbf{b}\right) \leq 0,
$$

then we prove that

$$
\lambda_{p}^{\prime \prime}\left(-c \mathfrak{D}_{x}+\mathcal{M}^{*}+\mathbf{b}\right) \leq \lambda_{p}\left(c \mathfrak{D}_{x}+\mathcal{M}+\mathbf{b}\right) .
$$

The existence of $\varphi$ will come as a side results of the proof of (6.3).

Step One : $\lambda_{p}\left(c \mathfrak{D}_{x}+\mathcal{M}+\mathbf{b}\right) \leq 0$. To prove (6.2), we argue by contradiction and assume that $\lambda_{p}\left(c \mathfrak{D}_{x}+\right.$ $\mathcal{M}+\mathbf{b})>0$.

Let $0<\rho<\lambda_{p}\left(c \mathfrak{D}_{x}+\mathcal{M}+\mathbf{b}\right)$ to be fixed later on. Then by definition of $v$ we have

$$
c \mathfrak{D}_{x}[v](x)+\mathcal{M}[v](x)+b(x) v(x)+\rho v(x) \geq \rho v(x)>0 \quad \text { for almost every } \quad x \in \mathbb{R} .
$$

Let $\iota>0$ be a small parameter to be fixed later on, and let us define $b_{\iota}(x):=\frac{f(x, v(x)+\iota)}{v(x)+\iota}$. Since $f$ satisfies ( $(\mathbf{H 4})$ and $(\mathbf{H 5})$, we have $b_{\iota}(x)<b(x)$ and there exists $C_{0}>0$ such that

$$
\left\|b_{\iota}-b\right\|_{\infty} \leq C_{0} \iota \text {. }
$$

Since $b_{\iota}(x) \geq b(x)-\left\|b_{\iota}-b\right\|_{\infty} \geq b(x)-C_{0} \iota$ by taking $\iota:=\frac{\rho}{2 C_{0}}$, then we easily check that $v$ then satisfies 


$$
c \mathfrak{D}_{x}[v](x)+\mathcal{M}[v](x)+b_{\iota}(x) v(x)+\rho v(x) \geq\left(\rho-C_{0} \iota\right) v \geq \frac{\rho}{2} v>0 \quad \text { for almost every } \quad x \in \mathbb{R} .
$$

Pick now $\zeta_{\tau} \in C_{c}^{\infty}(\mathbb{R})$ a positive symmetric mollifier whose support is include in $[-\tau, \tau]$ and define $v_{\tau}:=\zeta_{\tau} \star v(x)$. Then using the definition of $c \mathfrak{D}_{x}+\mathcal{M}$ and (6.1) we can check that $v_{\tau}$ satisfies

$$
c \mathfrak{D}_{x}\left[v_{\tau}\right](x)+\mathcal{M}\left[v_{\tau}\right](x)+b_{\iota}(x) v_{\tau}(x)+\rho v_{\tau}(x)+\int_{\mathbb{R}} \zeta_{\tau}(x-y)\left[b_{\iota}(y)-b_{\iota}(x)\right] v(y) d y \geq \frac{\rho}{2} v_{\tau}(x) .
$$

Recall that $v \in C^{0,1}(\mathbb{R})$ and $f(\cdot, s) \in C^{0,1}(\mathbb{R})$, since by definition $\inf _{\mathbb{R}}(v(x)+\iota)>0$ we have $b_{\iota} \in C^{0,1}(\mathbb{R})$ and therefore there exists $\kappa>0$ such that for all $x, y \in \mathbb{R}$

$$
\left|b_{\iota}(y)-b_{\iota}(x)\right| \leq \kappa|x-y| .
$$

As a consequence, since $\operatorname{supp}\left(\zeta_{\tau}\right) \subset[-\tau, \tau]$ we have

$$
\begin{aligned}
c \mathfrak{D}_{x}\left[v_{\tau}\right](x)+\mathcal{M}\left[v_{\tau}\right](x)+b_{\iota}(x) v_{\tau}(x)+\rho v_{\tau}(x) & \geq \frac{\rho}{2} v_{\tau}(x)-\left|\int_{\mathbb{R}} \zeta_{\tau}(x-y)\left[b_{\iota}(y)-b_{\iota}(x)\right] v(y) d y\right| \\
& \geq \frac{\rho}{2} v_{\tau}(x)-\kappa \int_{\mathbb{R}} \zeta_{\tau}(x-y)|x-y| v(y) d y \\
& \geq\left(\frac{\rho}{2}-2 \kappa \tau\right) v_{\tau}(x) .
\end{aligned}
$$

By choosing $\tau$ small enough, say $\tau \leq \tau_{0}:=\frac{\rho}{8 \kappa}$, we then achieve that

$$
c \mathfrak{D}_{x}\left[v_{\tau}\right](x)+\mathcal{M}\left[v_{\tau}\right](x)+b(x) v_{\tau}(x)+\rho v_{\tau}(x)>0 \quad \text { for all } \quad x \in \mathbb{R} .
$$

On the other hand since $b_{\iota}<b$, by Proposition 3.1 we have $\lambda_{p}\left(c \mathfrak{D}_{x}+\mathcal{M}+\mathbf{b}\right) \leq \lambda_{p}\left(c \mathfrak{D}_{x}+\mathcal{M}+\mathbf{b}_{\iota}\right)$ and therefore $\rho<\lambda_{p}\left(c \mathfrak{D}_{x}+\mathcal{M}+\mathbf{b}_{\iota}\right)$. So by definition of $\lambda_{p}$, there exists $\psi>0, \psi \in C^{1}(\mathbb{R})$ such that

$$
c \mathfrak{D}_{x}[\psi](x)+\mathcal{M}[\psi](x)+b_{\iota}(x) \psi(x)+\rho \psi(x)<0 \quad \text { for all } \quad x \in \mathbb{R} .
$$

Now let us recall that $f$ satisfies ( $(\mathbf{H 4})$, so there exists positive constant $R_{0}$ and $\nu>0$ such that $\frac{f(x, s)}{s}<-\nu$ for all $|x| \geq R_{0}, s \geq 0$. Let us now fixed $\rho:=\frac{1}{2} \min \left\{\nu, \lambda_{p}\left(c \mathfrak{D}_{x}+\mathcal{M}+\mathbf{b}_{\iota}\right)\right\}$ and on $\left[-R_{0}, R_{0}\right]$ let us define the following constant

$$
M:=\sup _{x \in\left(-R_{0}, R_{0}\right)} v_{\tau}(x) \quad m:=\inf _{x \in\left(-R_{0}, R_{0}\right)} \psi(x) .
$$

Next we consider the function $\Psi:=C \psi$ with $C:=\frac{M}{m}+1$. Since $v \in L^{1}(\mathbb{R}) \cap C^{0,1}(\mathbb{R}) v(x) \rightarrow 0$ as $|x| \rightarrow+\infty$ and so does $v_{\tau}$ therefore by using (6.4) and (6.5) and by definition of $C$, we then have

$$
\left\{\begin{array}{lll}
c \mathfrak{D}_{x}\left[v_{\tau}\right](x)+\mathcal{M}\left[v_{\tau}\right](x)+b_{\iota}(x) v_{\tau}(x)+\rho v_{\tau}(x)>0 & \text { for all } & x \in \mathbb{R} \\
c \mathfrak{D}_{x}[\Psi](x)+\mathcal{M}[\Psi](x)+b_{\iota}(x) \Psi(x)+\rho \Psi(x) \leq 0 & \text { for all } & x \in \mathbb{R} \\
v_{\tau}(x)<\Psi(x) & \text { for all } & x \in\left[-R_{0}, R_{0}\right], \\
0=\lim _{|x| \rightarrow \infty} v_{\tau}(x) \leq \lim _{|x| \rightarrow \infty} \Psi(x) . & &
\end{array}\right.
$$

By successively applying the weak and the strong comparison principle, Theorems 2.1 and 2.2, we then get $v_{\tau}<\Psi$ and we can define $\gamma^{*}:=\inf \left\{\gamma \geq 0 \mid v_{\tau} \leq \gamma \Psi\right\}$. Since for all $\gamma \geq 0$ the function $\gamma \Psi$ satisfies,

$$
c \mathfrak{D}_{x}[\gamma \Psi](x)+\mathcal{M}[\gamma \Psi](x)+b_{\iota}(x) \gamma \Psi(x)+\rho \gamma \Psi(x) \leq 0 \quad \text { for all } \quad x \in \mathbb{R},
$$

by a classical sweeping argument we then get that $\gamma^{*}=0$ and thus get the following contradiction

$$
0<v_{\tau} \leq 0 .
$$

Therefore (6.2) holds true, meaning that $\lambda_{p}\left(c \mathfrak{D}_{x}+\mathcal{M}+\mathbf{b}\right) \leq 0$. Let us now prove that the inequality (6.3) holds true as well. 
Step Two : $\lambda_{p}^{\prime \prime}\left(-c \mathfrak{D}_{x}+\mathcal{M}^{*}+\mathbf{b}\right) \leq \lambda_{p}\left(c \mathfrak{D}_{x}+\mathcal{M}+\mathbf{b}\right)$. Observe that in order to prove that (6.3) holds true, since by Proposition $3.4 \lambda_{p}\left(c \mathfrak{D}_{x}+\mathcal{M}+\mathbf{b}\right)=\lambda_{p}\left(-c \mathfrak{D}_{x}+\mathcal{M}^{*}+\mathbf{b}\right)$ and by the definition of $\lambda_{p}^{\prime \prime}$, it is then enough to prove the existence of $\varphi_{p} \in C^{1}(\mathbb{R}), \varphi_{p}$ a principal eigenfunction associated with $\lambda_{p}\left(-c \mathfrak{D}_{x}+\mathcal{M}^{*}+\mathbf{b}\right)$ such that $\varphi_{p} \in W^{1, \infty} \cap C^{1}(\mathbb{R})$. To do so, thanks to the a priori regularity provided by the equation, it is enough to show that such $\varphi_{p}$ exists and that $\varphi_{p} \in L^{\infty}$.

Consider now the increasing sequence $\left(R_{n}\right)_{n \in \mathbb{N}}:=\left(R_{0}+n\right)_{n \in \mathbb{N}}$ and the sequence $\lambda_{p}\left(-c \mathfrak{D}_{x}+\mathcal{M}_{R_{n}}^{*}+\mathbf{b}\right)$ associated to the operator $-c \mathfrak{D}_{x}+\mathcal{M}_{R_{n}}^{*}+\mathbf{b}$ defined in $\left(-R_{n}, R_{n}\right)$ for $\varphi \in C\left(\left[-R_{n}, R_{n}\right]\right) \cap C^{1}\left(\left(-R_{n}, R_{n}\right)\right)$. Let $\left(\varphi_{n}\right)_{n \in \mathbb{N}}$ be the sequence of positive principal eigenfunction associated with $\lambda_{p}\left(-c \mathfrak{D}_{x}+\mathcal{M}_{R_{n}}^{*}+\mathbf{b}\right)$. Such sequence $\left(\varphi_{n}\right)_{n \in \mathbb{N}}$ exists thanks to [31, 30] and moreover $\lambda_{p}\left(-c \mathfrak{D}_{x}+\mathcal{M}_{R_{n}}^{*}+\mathbf{b}\right) \rightarrow \lambda_{p}\left(-c \mathfrak{D}_{x}+\mathcal{M}^{*}+\mathbf{b}\right)$ as $n \rightarrow \infty$.

In addition, for all $n \geq 0, \varphi_{n}$ satisfies

$$
-c \mathfrak{D}_{x}\left[\varphi_{n}\right](x)+\mathcal{M}_{R_{n}}^{*}\left[\varphi_{n}\right](x)+\left(b(x)+\lambda_{p}\left(c \mathfrak{D}_{x}+\mathcal{M}_{R_{n}}+\mathbf{b}\right) \varphi_{n}=0 \quad \text { in }\left(-R_{n}, R_{n}\right) .\right.
$$

Let us recall that $b(x)<-\nu$ for all $|x|>R_{0}$. Therefore since by Proposition 3.4 and the previous step $\lambda_{p}\left(-c \mathfrak{D}_{x}+\mathcal{M}^{*}+\mathbf{b}\right)=\lambda_{p}\left(c \mathfrak{D}_{x}+\mathcal{M}+\mathbf{b}\right) \leq 0$ and since $\lambda_{p}\left(-c \mathfrak{D}_{x}+\mathcal{M}_{R_{n}}^{*}+\mathbf{b}\right) \rightarrow \lambda_{p}\left(-c \mathfrak{D}_{x}+\mathcal{M}^{*}+\mathbf{b}\right)$ there exists $R_{1}>0$ such that

$$
b(x)+\lambda_{p}\left(-c \mathfrak{D}_{x}+\mathcal{M}_{R_{n}}^{*}+\mathbf{b}\right) \leq-\frac{\nu}{4} \quad \text { for } \quad|x| \geq R_{1} .
$$

Let us now consider $\psi(x):=2 e^{-\alpha\left(|x|-R_{1}\right)}$ where $\alpha$ will be chosen later on. By a straightforward computation, we see that for all $R>R_{1}$ and $|x| \geq R_{1}$

$$
-c \mathfrak{D}_{x}[\psi](x)+\mathcal{M}_{R_{n}}^{*}[\psi](x)+\left(b(x)+\lambda_{p}\left(-c \mathfrak{D}_{x}+\mathcal{M}_{R_{n}}^{*}+\mathbf{b}\right)\right) \psi(x) \leq h(\alpha) \psi(x),
$$

with

$$
h(\alpha):=\left(|c| \alpha+\int_{\mathbb{R}} J(z) e^{\alpha|z|} d z-1-\frac{\nu}{4}\right) .
$$

Since $J$ is compactly supported, by the Lebesgue Theorem, the function $h$ is continuous and we can see that $h(0)=-\frac{\nu}{4}$. By assumption $\nu>0$, and by continuity of $h$ there exists $\alpha_{0}>0$ such that $h\left(\alpha_{0}\right)<0$. Thus, for $\alpha=\alpha_{0}$ we achieve

$$
-c \mathfrak{D}_{x}[\psi](x)+\mathcal{M}_{R_{n}}^{*}[\psi](x)+\left(b(x)+\lambda_{p}\left(-c \mathfrak{D}_{x}+\mathcal{M}_{R_{n}}^{*}+\mathbf{b}\right)\right) \psi(x) \leq 0 \quad \text { for } \quad|x| \geq R_{1} .
$$

Recall that by construction, the function $\varphi_{n}$ satisfies :

$$
-c \mathfrak{D}_{x}\left[\varphi_{n}\right](x)+\mathcal{M}_{R_{n}}^{*}\left[\varphi_{n}\right](x)+\left(b(x)+\lambda_{p}\left(-c \mathfrak{D}_{x}+\mathcal{M}_{R_{n}}^{*}+\mathbf{b}\right)\right) \varphi_{n}(x)=0 \quad \text { for } x \text { in } \quad\left(-R_{n}, R_{n}\right) .
$$

Since $R_{n} \rightarrow \infty$, there exists $n_{0}$ such that for all $n \geq n_{0} R_{n}>R_{1}$. Up to a rescaling, without loss of generality, we can assume that for all $n \geq n_{0}, \sup _{\left[-R_{1}, R_{1}\right]} \varphi_{n}=1$. Therefore for all $n \geq n_{0}$ we get

$$
\begin{array}{ll}
-c \mathfrak{D}_{x}\left[\varphi_{n}\right](x)+\mathcal{M}_{R_{n}}^{*}\left[\varphi_{n}\right](x)+\left(b(x)+\lambda_{p}\left(-c \mathfrak{D}_{x}+\mathcal{M}_{R_{n}}^{*}+\mathbf{b}\right)\right) \varphi_{n}(x)=0 & \text { for all } \quad x \in\left(-R_{n}, R_{n}\right) \\
-c \mathfrak{D}_{x}[\psi](x)+\mathcal{M}_{R_{n}}^{*}[\psi](x)+\left(b(x)+\lambda_{p}\left(-c \mathfrak{D}_{x}+\mathcal{M}_{R_{n}}^{*}+\mathbf{b}\right)\right) \psi(x) \leq 0 & \text { for all } \quad|x| \geq R_{1} \\
\varphi_{n}<\psi(x) & \text { for all } \quad x \in\left[-R_{1}, R_{1}\right]
\end{array}
$$

We claim that

Claim 6.2. For all $n \geq n_{0}$, then $\varphi_{n} \leq \psi$.

Assume for the moment the claim holds true then we can readily finish our proof by arguing as follows. Since $\varphi_{n} \leq \psi<2 e^{\alpha_{0} R_{1}}$, by using the local regularity we can see that the sequence $\left(\varphi_{n}\right)_{n \in \mathbb{N}}$ is bounded uniformly in $C^{1,1}(\mathbb{R})$. Therefore by using a diagonal extraction and that $\sup \varphi_{n}=1$, from the sequence $\left(\varphi_{n}\right)_{n \in \mathbb{N}}$ we can extract a subsequence that converges to a nontrivial smooth function $\varphi \geq \not \equiv$ in the $C_{l o c}^{1}(\mathbb{R})$ topology. Moreover, $\varphi \leq \psi$ satisfies

$$
-c \mathfrak{D}_{x}[\varphi]+\mathcal{M}^{*}[\varphi](x)+\left(b(x)+\lambda_{p}\left(-c \mathfrak{D}_{x}+\mathcal{M}^{*}+\mathbf{b}\right)\right) \varphi(x)=0 \quad \text { for all } \quad x \in \mathbb{R},
$$

which since $\lambda_{p}\left(-c \mathfrak{D}_{x}+\mathcal{M}^{*}+\mathbf{b}\right) \leq 0$ enforces

$$
-c \mathfrak{D}_{x}[\varphi](x)+\mathcal{M}^{*}[\varphi](x)+b(x) \varphi(x) \geq 0 \quad \text { for all } \quad x \in \mathbb{R} .
$$


By using the strong maximum principle, we see that $\varphi>0$ and from the local regularity since $\varphi \in L^{\infty}$ we see that $\varphi \in W^{1, \infty}$. Hence $\left(\varphi, \lambda_{p}\left(c \mathfrak{D}_{x}+\mathcal{M}+\mathbf{b}\right)\right)$ belongs to the set of test function that define $\lambda_{p}^{\prime \prime}\left(c \mathfrak{D}_{x}+\mathcal{M}+\mathbf{b}\right)$ and therefore

$$
\lambda_{p}^{\prime \prime}\left(-c \mathfrak{D}_{x}+\mathcal{M}^{*}+\mathbf{b}\right) \leq \lambda_{p}\left(-c \mathfrak{D}_{x}+\mathcal{M}^{*}+\mathbf{b}\right)=\lambda_{p}\left(c \mathfrak{D}_{x}+\mathcal{M}+\mathbf{b}\right),
$$

proving that (6.3) holds true. We get similarly $\varphi \in W^{1,1}(\mathbb{R})$ by observing that $\varphi \in L^{1}(\mathbb{R})$ since $0 \leq \varphi \leq \psi$ and $\psi \in L^{1}$.

Let us now prove the claim.

Proof of the claim. Recall that $\varphi_{n} \in C^{1}\left(\left(-R_{n}, R_{n}\right)\right) \cap L^{\infty}\left(\left(-R_{n}, R_{n}\right)\right)$ and $\inf _{\left(-R_{n}, R_{n}\right)} \psi>0$. Therefore, we can find $\gamma_{0}>0$ such that $\varphi_{n} \leq \gamma_{0} \psi$ and therefore the following quantity is then well defined:

$$
\gamma^{*}:=\inf \left\{\gamma \geq 0 \mid \varphi_{n} \leq \gamma \psi\right\}
$$

We will show that $\gamma^{*} \leq 1$. Assume by contradiction that $\gamma^{*}>1$. By definition of $\gamma^{*}$ and since $\varphi_{n}$ and $\psi$ are continuous in $\left[-R_{n}, R_{n}\right]$ we have $\varphi_{n} \leq \gamma^{*} \psi$ in $\left[-R_{n}, R_{n}\right]$ and there exists $x_{0} \in\left[-R_{n}, R_{n}\right]$ such that $\varphi_{n}\left(x_{0}\right)=\gamma^{*} \psi\left(x_{0}\right)$. Since $\gamma^{*}>1$ and $\varphi_{n}<\psi$ in $\left[-R_{1}, R_{1}\right]$, we deduce that $x_{0} \in\left[-R_{n}, R_{n}\right] \backslash\left[-R_{1}, R_{1}\right]$. Since $\varphi_{n} \leq \gamma^{*} \psi$, by the strong maximum principle we then infer that $\varphi_{n}<\gamma^{*} \psi$ in $\left(-R_{n}, R_{n}\right)$ and thus $x_{0}= \pm R_{n}$. Since $-c<0$, thanks to Theorems 3.2 we have $\varphi_{n}\left(-R_{n}\right)=0<\gamma^{*} \psi\left(-R_{n}\right)$, so we must have $x_{0}=+R_{n}$. By using now (6.9) and (6.8), we can check that the function $w:=\gamma^{*} \psi-\varphi_{n}$ satisfies

$$
-c \mathfrak{D}_{x}[w](x)+\mathcal{M}_{R_{n}}^{*}[w](x)+\left(b(x)+\lambda_{p}\left(c \mathfrak{D}_{x}+\mathcal{M}_{R_{n}}+\mathbf{b}\right) w<0 \quad \text { for all } \quad|x|>R_{1}\right.
$$

Since $w\left(R_{n}\right)=0$, and since $w$ is continuous in $\left[-R_{n}, R_{n}\right]$ we then infer that

$$
\liminf _{x \rightarrow R_{n}} \mathfrak{D}_{x}[w]=\lim _{x \rightarrow R_{n}}-\frac{1}{c} \int_{-R_{n}}^{R_{n}} J(x-y) w(y) d y>0,
$$

which since $w\left(R_{n}\right)=0$ implies that there exists $x_{2} \in\left(-R_{n}, R_{n}\right)$ such that $w\left(x_{2}\right)<0$ contradicting that $w \geq 0$ in $\left[-R_{n}, R_{n}\right]$. Hence $\gamma^{*} \leq 1$ and thus $\varphi_{n} \leq \psi$.

6.3. The final argument. We show that the equation (1.5) has a unique solution. To do so let us argue by contradiction and assume that there is another solution $u_{2}$. From the above subsection, we know that $u_{2} \in L^{1}$. Let us denote $v(x):=\sup \left(u(x), u_{2}(x)\right)$, then from the definition of $v$, we deduce that $v$ is a weak sub-solution to (1.5), that is we have

$$
c \mathfrak{D}_{x}[v](x)+\mathcal{M}[v](x)+f(x, v)(x) \geq 0 \quad \text { for almost every } \quad x \in \mathbb{R} .
$$

As above set $b(x):=\frac{f(x, v(x))}{v(x)}$ and now let us consider the operator $-c \mathfrak{D}_{x}+\mathcal{M}^{*}+\mathbf{b}$.

We claim that

Claim 6.3. There exists a sequences $\left(\delta_{n}\right)_{n \in \mathbb{N}}$ and $\left(\psi_{n}\right)_{n \in \mathbb{N}}$ and a smooth bounded non trivial function $\psi \geq 0$ such that:

i) for all $n \geq 0, \delta_{n}>0, \delta_{n+1} \leq \delta_{n}$, and $\lim _{n \rightarrow \infty} \delta_{n}=0$

ii) for all $n \geq 0, \psi_{n} \geq 0, \psi_{n} \in C^{k}(\mathbb{R}) \cap W^{1, \infty}(\mathbb{R})$, and $\quad \psi_{n} \rightarrow \psi \quad$ in $C_{\text {loc }}^{k, \alpha}(\mathbb{R})$

iii) for all $n \geq 0$

$$
-c \mathfrak{D}_{x}\left[\psi_{n}\right](x)+\mathcal{M}^{*}\left[\psi_{n}\right](x)+b(x) \psi_{n}(x)+\delta_{n} \psi_{n} \geq 0 \quad \text { for all } \quad x \in \mathbb{R} .
$$

Assume for the moment that the claim holds true, then we finish our proof of the uniqueness by arguing as follows. Let us multiply by $u$ the equation satisfied by $\psi_{n}$ and integrate it over $\mathbb{R}$, the integration is licit since $u \in C^{1}(\mathbb{R}) \cap H^{1}(\mathbb{R})$ and $\psi_{n} \in W^{1, \infty}(\mathbb{R})$. We then get

Therefore

$$
\mathfrak{I}_{\mathfrak{n}}:=-c \int_{\mathbb{R}} u \mathfrak{D}_{x}\left[\psi_{n}\right]+\int_{\mathbb{R}} u \mathcal{M}\left[\psi_{n}\right]+\int_{\mathbb{R}} b(x) u \psi_{n}+\delta_{n} \int_{\mathbb{R}} u \psi_{n}(x) \geq 0 .
$$

$$
\liminf _{n \rightarrow \infty} \mathfrak{I}_{\mathfrak{n}} \geq 0
$$


Since $\psi_{n} \in W^{1, \infty}$ and $u \in H^{1}(\mathbb{R})$ by using integration by parts, Fubini's Theorems and the equation satisfied by $u$, we can check that

$$
\begin{aligned}
\mathfrak{I}_{\mathfrak{n}} & =c \int_{\mathbb{R}} \mathfrak{D}_{x}[u] \psi_{n}+\int_{\mathbb{R}} \psi_{n} \mathcal{M}[u]+\int_{\mathbb{R}} b(x) u \psi_{n}+\delta_{n} \int_{\mathbb{R}} u \psi_{n} \\
& =\int_{\mathbb{R}}\left(\frac{f(x, v(x))}{v(x)}-\frac{f(x, u(x))}{u(x)}\right) u \psi_{n}+\delta_{n} \int_{\mathbb{R}} u \psi_{n} .
\end{aligned}
$$

Since $\delta_{n} \rightarrow 0, u_{\varepsilon} \in L^{1}, \psi \in L^{\infty}$ and $\psi_{n} \rightarrow \psi$ pointwise we get that

$$
\limsup _{n \rightarrow \infty} I_{n}=\int_{\mathbb{R}}\left(\frac{f(x, v(x))}{v(x)}-\frac{f(x, u(x))}{u(x)}\right) u \psi,
$$

which using that $u \leq \not \equiv v$ and $f(x, s) / s$ is decreasing implies that

$$
\int_{\mathbb{R}}\left(\frac{f(x, v(x))}{v(x)}-\frac{f(x, u(x))}{u(x)}\right) u \psi<0
$$

and thus

$$
\limsup _{n \rightarrow \infty} \mathfrak{I}_{\mathfrak{n}}<0
$$

By combining the later estimate with (6.11) we get the following contradiction

$$
0 \leq \liminf _{n \rightarrow \infty} \mathfrak{I}_{\mathfrak{n}} \leq \limsup _{n \rightarrow \infty} \mathfrak{I}_{\mathfrak{n}}<0 .
$$

Hence $u \equiv v \equiv u_{2}$.

Proof of the Claim. Thanks to Proposition 6.1 we know that $\lambda_{p}^{\prime \prime}\left(-c \mathfrak{D}_{x}+\mathcal{M}^{*}+\mathbf{b}\right) \leq 0$. Let us now analyse two separate possibility either $\lambda_{p}^{\prime \prime}\left(-c \mathfrak{D}_{x}+\mathcal{M}^{*}+\mathbf{b}\right)=0$ or $\lambda_{p}^{\prime \prime}\left(-c \mathfrak{D}_{x}+\mathcal{M}^{*}+\mathbf{b}\right)<0$. In the later situation, the existence of the sequences is straightforward. Indeed from the definition of $\lambda_{p}^{\prime \prime}$ there exists a test function $\psi \geq 0, \psi \in C^{1}(\mathbb{R}) \cap W^{1, \infty}(\mathbb{R})$ such that $-c \mathfrak{D}_{x}[\psi]+\mathcal{M}^{*}[\psi]+b(x) \psi \geq 0$. We achieve i), ii) and iii) by taking the sequence $\left(\psi_{n}\right)_{n \in \mathbb{N}}$ defined for all $n$ by $\psi_{n}=\psi$, and any decreasing sequence $\left(\delta_{n}\right)_{n}$ that converges to 0 .

Let us now look at the situation $\lambda_{p}^{\prime \prime}\left(-c \mathfrak{D}_{x}+\mathcal{M}^{*}+\mathbf{b}\right)=0$. In this situation, let $\left(\lambda_{n}\right)_{n \in \mathbb{N}}$ be a monotone decreasing sequence of positive numbers such that $\lambda_{n} \rightarrow 0$ as $n \rightarrow \infty$. By definition of $\lambda_{p}^{\prime \prime}$, for each $n$ there exists $\psi_{n}$ such that

$$
-c \mathfrak{D}_{x}\left[\psi_{n}\right](x)+\mathcal{M}^{*}\left[\psi_{n}\right](x)+b(x) \psi_{n}+\lambda_{n} \psi_{n}(x) \geq 0 \quad \text { for all } \quad x \in \mathbb{R} .
$$

By assumption for $n$ large enough, says $n \geq n_{0}$, we have $\lambda_{n}<\nu / 2$ and thus $b(x)+\lambda_{n} \leq-\frac{\nu}{2}$ for $|x| \geq R_{0}$. So by integrating (6.13) over $(-R, R)$, we then get

$$
-c \int_{-R}^{R} \mathfrak{D}_{x}\left[\psi_{n}\right]+\int_{-R}^{R} \mathcal{M}^{*}\left[\psi_{n}\right] \geq \frac{\nu}{2} \int_{-R}^{R} \psi_{n}(x)-C_{n},
$$

with $C_{0}:=\left(\frac{\nu}{2}+\sup _{x \in\left[R_{0}, R_{0}\right]}\left\|b(x)+\lambda_{n}\right\|_{\infty}\right) \int_{-R_{0}}^{R_{0}} \psi_{n}$.

After integration, since $\psi_{n} \in L^{\infty}$, there exists $C_{n}$ such that

$$
C_{n} \geq \frac{\nu}{2} \int_{-R}^{R} \psi_{n}
$$

Therefore, $\psi_{n} \in L^{1}(\mathbb{R})$ and as a consequence $\psi_{n}(x) \rightarrow 0$ as $|x| \rightarrow+\infty$.

Now by arguing as in the proof of Proposition 6.1, we can also find $\alpha>0$ independent of $n$ such that for all $C>0$, the function $w:=C e^{-\alpha|x|}$ satisfies for all $|x| \geq R_{0}$

$$
-c \mathfrak{D}_{x}[w]+\mathcal{M}^{*}[w]+b(x) w+\lambda_{n} w+\frac{\nu}{4} w<0 .
$$


Let us now normalised $\psi_{n}$ such that $\sup _{x \in\left[-R_{0}, R_{0}\right]} \psi_{n}(x)=1$ and take $C:=2 e^{\alpha R_{0}}$. By our choice of parameter, we have $w \geq \psi_{n}$ on $\left[-R_{0}, R_{0}\right]$. Now since for $n \geq n_{0} b(x)+\lambda_{n} \leq 0$ for $|x| \geq R_{0}$ by repeating the argument used in the proof of Proposition 6.1 we see that for all $n \geq n_{0}$, we then have $\psi_{n} \leq w$ in $\mathbb{R}$.

Therefore the sequence $\left(\psi_{n}\right)_{n \in \mathbb{N}}$ is uniformly bounded in $L^{\infty}(\mathbb{R}) \cap C_{l o c}^{1}(\mathbb{R})$, and as a consequence by a diagonal extraction we can extract a converging subsequence, that is there exists $\psi \geq \not \equiv 0$ such that $\psi_{n} \rightarrow \psi$ in $C_{l o c}^{1}(\mathbb{R})$. Moreover we can check that $\psi \in L^{\infty}$ satisfies $-c \mathfrak{D}_{x}[\psi]+\mathcal{M}^{*}[\psi]+b(x) \psi=0$ and $\sup \psi=1$. Hence $\left(\psi_{n}\right)_{n \in \mathbb{N}},\left(\lambda_{n}\right)_{n \in \mathbb{N}}$ are our desired sequence. $\left[-R_{0}, R_{0}\right]$

\section{Non-EXistence of A SOLUtion}

In this section, we deal with the non-existence of positive solution to (1.5) when $\lambda_{p}\left(c \mathfrak{D}_{x}+\mathcal{M}+\mathbf{f}_{\mathbf{s}}(\mathbf{x}, \mathbf{0})\right) \geq$ 0 , proving the necessary condition stated in Theorem 1.1 validating that the sign of this quantity is the right criteria in order to predict the survival of the population. To simplify the presentation of the proofs, we treat the two cases: $\lambda_{p}\left(c \mathfrak{D}_{x}+\mathcal{M}+\mathbf{f}_{\mathbf{s}}(\mathbf{x}, \mathbf{0})\right)>0$ and $\lambda_{p}\left(c \mathfrak{D}_{x}+\mathcal{M}+\mathbf{f}_{\mathbf{s}}(\mathbf{x}, \mathbf{0})\right)=0$ separately, the proof in the second case being more involved.

Case $\lambda_{p}\left(c \mathfrak{D}_{x}+\mathcal{M}+\mathbf{f}_{\mathbf{s}}(\mathbf{x}, \mathbf{0})\right)>0$ : In this situation we argue as follows. Assume by contradiction that a positive bounded solution $u$ exists. By assumption, $u$ satisfies

$$
c \mathfrak{D}_{x}[u]+\mathcal{M}[u]+\frac{f(x, u)}{u} u=0 \quad \text { in } \quad \mathbb{R} .
$$

From Proposition 6.1 we deduce that $\lambda_{p}\left(c \mathfrak{D}_{x}+\mathcal{M}+\frac{\mathbf{f}(\mathbf{x}, \mathbf{u})}{\mathbf{u}}\right) \leq 0$. Therefore since $f(x, u) / u \leq f_{s}(x, 0)$, by using the monotonic property of $\lambda_{p}$ with respect to the potential, we see that

$$
\lambda_{p}\left(c \mathfrak{D}_{x}+\mathcal{M}+\mathbf{f}_{\mathbf{s}}(\mathbf{x}, \mathbf{0})\right) \leq \lambda_{p}\left(c \mathfrak{D}_{x}+\mathcal{M}+\frac{\mathbf{f}(\mathbf{x}, \mathbf{u})}{\mathbf{u}}\right) \leq 0 .
$$

Thus, we then obtain an obvious contradiction since $\lambda_{p}\left(c \mathfrak{D}_{x}+\mathcal{M}+\mathbf{f}_{\mathbf{s}}(\mathbf{x}, \mathbf{0})\right)>0$.

Case $\lambda_{p}\left(c \mathfrak{D}_{x}+\mathcal{M}+\mathbf{f}_{\mathbf{s}}(\mathbf{x}, \mathbf{0})\right)=0$ : To treat this case, we will adapt to our situation an argument introduced in [5] in the case $c=0$. We argue again by contradiction. Assume that a non-negative, non identically zero, bounded solution $u$ exists. By a straightforward application of the maximum principle, since $u \neq \equiv 0$ we have $u>0$ in $\mathbb{R}$. Set $a(x):=f_{s}(x, 0)$ and $b(x):=\frac{f(x, u(x))}{u(x)}$, then by Propositions 6.1 and 3.1 we have

$$
0=\lambda_{p}\left(c \mathfrak{D}_{x}+\mathcal{M}+\mathbf{a}\right) \leq \lambda_{p}\left(c \mathfrak{D}_{x}+\mathcal{M}+\mathbf{b}\right) \leq 0 .
$$

Therefore we have

$$
\lambda_{p}\left(c \mathfrak{D}_{x}+\mathcal{M}+\mathbf{a}\right)=\lambda_{p}\left(c \mathfrak{D}_{x}+\mathcal{M}+\mathbf{b}\right)=0 .
$$

Fix $R_{0}>0$ and let us denote $\zeta \in C(\mathbb{R})$ a smooth regularisation of $\chi_{R_{0} / 2}$ the characteristic function of the interval $\left(-\frac{R_{0}}{2}, \frac{R_{0}}{2}\right)$. Since $b<a$, we can find $\varepsilon_{0}>0$ small enough such that $b \leq b+\varepsilon_{0} \zeta<a$.

By Proposition 3.1 and (7.2) then have

$$
\lambda_{p}\left(c \mathfrak{D}_{x}+\mathcal{M}+\mathbf{b}+\varepsilon_{\mathbf{0}} \zeta\right)=0 .
$$
have

Since by Theorem 1.2 we can check that $\lambda_{p}\left(-c \mathfrak{D}_{x}+\mathcal{M}+\mathbf{b}+\varepsilon_{\mathbf{0}} \zeta\right)=\lambda_{p}\left(c \mathfrak{D}_{x}+\mathcal{M}+\mathbf{b}+\varepsilon_{\mathbf{0}} \zeta\right)$, we then

$$
\lambda_{p}\left(-c \mathfrak{D}_{x}+\mathcal{M}+\mathbf{b}+\varepsilon_{\mathbf{0}} \zeta\right)=0 .
$$

Now thanks to Proposition 6.1 there exists $\varphi>0, \varphi \in C^{1}(\mathbb{R}) \cap W^{1,1}(\mathbb{R}) \cap W^{1, \infty}(\mathbb{R})$ and sequences $\left(\psi_{n}\right)_{n \in \mathbb{N}},\left(\delta_{n}\right)_{n \in \mathbb{N}}$ and a positive function $\psi \in L^{\infty}$ such that

i)

$$
-c \mathfrak{D}_{x}[\varphi](x)+\mathcal{M}[\varphi](x)+b(x) \varphi(x) \geq 0 \quad \text { for all } \quad x \in \mathbb{R} .
$$

ii) for all $n \geq 0, \delta_{n}>0, \delta_{n+1} \leq \delta_{n}$, and $\lim _{n \rightarrow \infty} \delta_{n}=0$

iii) for all $n \geq 0, \psi_{n}>0, \psi_{n} \in C^{1}(\mathbb{R}) \cap W^{1, \infty}(\mathbb{R})$, and $\quad \psi_{n} \rightarrow \psi \quad$ in $C_{\text {loc }}^{1, \alpha}(\mathbb{R})$ 
iv) for all $n \geq 0$

$$
-c \mathfrak{D}_{x}\left[\psi_{n}\right](x)+\mathcal{M}^{*}\left[\psi_{n}\right](x)+\left(b(x)+\varepsilon_{0} \zeta(x)\right) \psi_{n}(x)+\delta_{n} \psi_{n} \geq 0 \quad \text { for all } \quad x \in \mathbb{R} .
$$

Arguing now as in the final argument subsection of the section 6 we achieve the contradiction

$$
0=-\varepsilon_{0} \int_{\mathbb{R}} \varphi(x) \psi(x) \zeta(x) d x<0 .
$$

\section{LONG TIME BeHAVIOUR}

In this section, we investigate the long-time behaviour of the positive solution $u(t, x)$ of

$$
\begin{aligned}
& \partial_{t} u(t, x)=\mathcal{M}[u](t, x)+f(x-c t, u(t, x)) \quad \text { for } t>0, \text { and } x \in \mathbb{R}, \\
& u(0, x)=u_{0}(x) \text { in } \mathbb{R} .
\end{aligned}
$$

For any $u_{0} \in C^{k}(\mathbb{R}) \cap L^{\infty}$ or in $C^{k}(\mathbb{R}) \cap L^{1}\left(\mathbb{R}^{N}\right)$ the existence of a smooth solution $u(t, x) \in C^{1}\left((0,+\infty), C^{\min \{1, k\}}(\mathbb{R})\right)$ respectively $u(t, x) \in C^{1}\left((0,+\infty), C^{\min \{1, k\}}(\mathbb{R}) \cap L^{1}(\mathbb{R})\right)$ is a straightforward consequence of the CauchyLipschitz Theorem and of the KPP structure of the nonlinearity $f$. Note also that in the moving frame of speed $c$ the smooth solution $u_{c}(t, x):=u(t, x+c t)$ will be also a solution of the following problem

$$
\begin{aligned}
& \partial_{t} u_{c}(t, x)=\mathcal{M}\left[u_{c}\right](t, x)+c \mathfrak{D}_{x}\left[u_{c}\right](t, x)+f\left(x, u_{c}(t, x)\right) \text { for } t>0, \text { and } x \in \mathbb{R}, \\
& u_{c}(0, x)=u_{0}(x) \text { in } \mathbb{R} .
\end{aligned}
$$

Observe that the function $\tilde{u}(t, x):=u_{c}(t, x-c t)$ is also a solution to (8.1) so by uniqueness of the solution of the Cauchy problem, we deduce that $u(t, x)=\tilde{u}_{c}(t, x)=u_{c}(t, x-c t)$.

Before going to the proof of the asymptotic behaviour, let us recall some useful results

Lemma 8.1. Assume that $u_{0}$ is a sub-solution to (8.3), then the solution $u_{c}(t, x)$ is increasing in time. Conversely, if $u_{0}$ is a super-solution to (8.3) then $u_{c}(t, x)$ is decreasing in time.

The proof of this Lemma follows from a straightforward application of the parabolic maximum principle and is left to reader. Note that it may happen that $u_{0}$ is sub-( super-) solution of (8.1) but not of (8.3) and vice versa.

Let us now prove the asymptotic behaviour of the solution of (1.4) and finish the proof of Theorem 1.1. We split our analysis into two main steps, first we establish a local uniform convergence towards the steady states of the system then by adapting an argument used in [13, 4] to our situation we prove the uniform convergence.

8.1. Step One : Local uniform convergence. Let us first prove that for any bounded and smooth $u_{0}$ then the solution $u_{c}(t, x)$ of (8.3) converges locally uniformly to $\bar{u}_{c}(x)$ a stationary solution of (8.3).

Depending on the sign of $\lambda_{p}\left(c \mathfrak{D}_{x}+\mathcal{M}+\mathbf{a}\right)$ this stationary solution will be either 0 or the unique non trivial solution of (8.3). So, let $z(t, x)$ be the solution of

$$
\begin{aligned}
& \partial_{t} z(t, x)=\mathcal{M}[z](t, x)+f(x-c t, z(t, x)) \quad \text { for } t>0, \text { and } x \in \mathbb{R}, \\
& z(0, x)=C\left\|u_{0}\right\|_{\infty} \text { on } \mathbb{R} .
\end{aligned}
$$

Since $S(x) \in L^{\infty}$ by choosing $C$ large enough, the constant $C\left\|u_{0}\right\|_{\infty}$ is a super-solution of (8.1). Note that since $z(0, x) \equiv C$ ste, then it is also a super-solution of (8.3). Therefore both $z$ and $z_{c}(t, x)$ are decreasing function of $t$ and by the parabolic maximum principle $u(t, x) \leq z(t, x)$ for all $(t, x) \in[0,+\infty) \times \mathbb{R}$ and $u_{c}(t, x) \leq z_{c}(t, x)$ for all $(t, x) \in[0,+\infty) \times \mathbb{R}$. Therefore,

$$
\begin{array}{cl}
\limsup _{t \rightarrow \infty} u(t, x) \leq \limsup _{t \rightarrow \infty} z(t, x) \quad \text { for all } & x \in \mathbb{R} . \\
\limsup _{t \rightarrow \infty} u_{c}(t, x) \leq \limsup _{t \rightarrow \infty} z_{c}(t, x) \quad \text { for all } & x \in \mathbb{R} .
\end{array}
$$


Since $z_{c}(t, x)$ is a decreasing function of $t$, and $z_{c} \geq 0$, we get $\lim _{t \rightarrow \infty} z_{c}(t, x)=\bar{z}(x)$, for all $x \in \mathbb{R}$. Moreover by using standard regularity estimates $z_{c}(t, x)$ converges to $\bar{z}$ in $C_{l o c}^{1, \alpha}(\mathbb{R})$ topology and thus $\bar{z}$ is a bounded stationary solution of (8.3). By uniqueness of the positive stationary solution, we conclude that $\bar{z}=\bar{u}_{c}$.

Therefore we have

$$
\limsup _{t \rightarrow \infty} u_{c}(t, x) \leq \bar{u}_{c}(x) \text { for all } \quad x \in \mathbb{R} .
$$

When $\lambda_{p}\left(c \mathfrak{D}_{x}+\mathcal{M}+\mathbf{a}\right) \geq 0$, then no non trivial solution exists and therefore we have $\bar{u}_{c} \equiv 0$ and $u(t, x)$ as well as $u_{c}(t, x)$ converges locally uniformly to 0 . Let us now prove that when $\lambda_{p}\left(c \mathfrak{D}_{x}+\mathcal{M}+\mathbf{a}\right)<0$, then $\bar{u}_{c}>0$ and $u(t, x) \rightarrow \bar{u}_{c}$ locally uniformly as $t \rightarrow+\infty$.

In this situation, from Subsection 4.1, thanks to Remark 5, there exists $R_{0}>0, \psi \in C^{2}\left(-R_{0}, R_{0}\right) \cap$ $C\left(\left[-R_{0}, R_{0}\right]\right)$ such that for all $x \in\left(-R_{0}, R_{0}\right)$ we have

$$
c \mathfrak{D}_{x}[\psi]+\mathcal{M}_{R_{0}}[\psi]+\left(a(x)+\lambda_{p}\left(c \mathfrak{D}_{x}+\mathcal{M}_{R_{0}}+\mathbf{a}\right)+\delta\right) \psi \geq \frac{d^{*}}{4}>0
$$

with $\left.\lambda_{p}\left(c \mathfrak{D}_{x}+\mathcal{M}_{R_{0}}+\mathbf{a}\right)+\delta\right)<\frac{\lambda_{p}\left(c \mathfrak{D}_{x}+\mathcal{M}_{R_{0}}+\mathbf{a}\right)}{2}<\frac{\lambda_{p}\left(c \mathfrak{D}_{x}+\mathcal{M}_{R_{0}}+\mathbf{a}\right)}{4}$.

Set $\left.\gamma:=\lambda_{p}\left(c \mathfrak{D}_{x}+\mathcal{M}_{R_{0}}+\mathbf{a}\right)+\delta\right)$ and let us extend $\psi$ continuously by zero outside $\left(-R_{0}, R_{0}\right)$, and let us denote $\bar{\psi}$ this extension. One one hand since by definition $\bar{\psi} \in C^{0,1}(\mathbb{R}) \cap C^{2}\left(\left(-\infty, R_{0}\right)\right)$ we have $\bar{\psi} \equiv 0$ in $\mathbb{R} \backslash\left(-R_{0}+\tau, R_{0}\right]$ it follows that

$$
c \mathfrak{D}_{x}[\bar{\psi}]+\mathcal{M}[\bar{\psi}]+(a(x)+\gamma) \bar{\psi}=\int_{\mathbb{R}} J(x-y) \bar{\psi}(y) d y \geq 0 \quad \text { for all } \quad x \in \mathbb{R} \backslash\left(-R_{0}+\tau, R_{0}\right] .
$$

On the other hand, since $\bar{\psi} \geq 0$ and $\mathcal{M}[\bar{\psi}] \geq \mathcal{M}_{R_{0}}[\bar{\psi}]$, we then have

$$
c \mathfrak{D}_{x}[\bar{\psi}]+\mathcal{M}[\bar{\psi}]+(a(x)+\gamma) \bar{\psi} \geq \frac{d^{*}}{4}>0 \quad \text { for all } \quad x \in\left(-R_{0}, R_{0}\right)
$$

Therefore, $\bar{\psi}$ satisfies

$$
c \mathfrak{D}_{x}[\bar{\psi}]+\mathcal{M}[\bar{\psi}]+(a(x)+\gamma) \bar{\psi} \geq 0 \quad \text { for almost every } \quad x \in \mathbb{R}
$$

Pick now $\zeta_{\iota} \in C_{c}^{\infty}(\mathbb{R})$ a positive symmetric mollifier whose support is include in $[-\iota, \iota]$ and define $\psi_{\iota}:=\zeta_{\iota} \star \bar{\psi}$.

Then from the above equation we can check that $\psi_{\iota}$ satisfies for all $x \in \mathbb{R}$

$$
c \mathfrak{D}_{x}\left[\psi_{\iota}\right]+\mathcal{M}\left[\psi_{\iota}\right]+(a(x)+\gamma) \psi_{\iota}+\int_{\mathbb{R}} \zeta_{\tau}(x-y)[a(y)-a(x)] \bar{\psi}(y) d y \geq 0 .
$$

By using that $a \in C^{0, \alpha}(\mathbb{R})$, there exists $L_{0}$ such that for all $x, y \in \mathbb{R}$, we have $|a(x)-a(y)| \leq L_{0}|x-y|^{\alpha}$ and therefore from the above inequality we deduce that

$$
c \mathfrak{D}_{x}\left[\psi_{\iota}\right]+\mathcal{M}\left[\psi_{\iota}\right]+\left(a(x)+\frac{\gamma}{2}\right) \psi_{\iota} \geq\left(-\frac{\gamma}{2}-2 L_{0} \iota^{\alpha}\right) \psi_{\iota}
$$

Then by taking $\iota$ small enough, says $\iota \leq \iota_{0}:=\left(\frac{-\gamma}{4 L_{0}}\right)^{\frac{1}{\alpha}}$ we then achieve for all $x \in \mathbb{R}$.

$$
c \mathfrak{D}_{x}\left[\psi_{\iota}\right]+\mathcal{M}\left[\psi_{\iota}\right]+\left(a(x)+\frac{\gamma}{2}\right) \psi_{\iota} \geq 0 .
$$

Let us now check that for $\kappa$ small, then $\kappa \psi_{\iota}$ is a subsolution to 8.3). Indeed, thank to the regularity of $f$ and since $\psi_{\iota}$ is bounded and $f(x, 0)=0$, we can find $\kappa^{*}$ such that for all $\kappa \leq \kappa^{*}$ we have for all $x \in \mathbb{R}$

$$
\left|\frac{f\left(x, \kappa \psi_{\iota}\right)}{\kappa \psi_{\iota}}-f_{s}(x, 0)\right| \leq-\frac{\gamma}{4} .
$$

Now observe that since $f(x, 0)=0$, when $\psi_{\iota}=0$, we trivially have

$$
c \mathfrak{D}_{x} \kappa \psi_{\iota}+\mathcal{M}\left[\kappa \psi_{\iota}\right]+f\left(x, \kappa \psi_{\iota}\right)=\mathcal{M}\left[\psi_{\iota}\right] \geq 0
$$


whereas for $x$ such that $\psi_{\iota}>0$ by definition we have

$$
c \mathfrak{D}_{x} \kappa \psi_{\iota}+\mathcal{M}\left[\kappa \psi_{\iota}\right]+f\left(x, \kappa \psi_{\iota}\right)=\left(\left[\frac{f\left(x, \kappa \psi_{\iota}\right)}{\kappa \psi_{\iota}}-f_{s}(x, 0)\right]-\frac{\gamma}{2}\right) \psi_{\iota} \geq-\frac{\gamma \kappa}{4} \psi_{\iota}>0 .
$$

Therefore

$$
c \mathfrak{D}_{x} \kappa \psi_{\iota}+\mathcal{M}\left[\kappa \psi_{\iota}\right]+f\left(\xi, \kappa \psi_{\iota}\right) \geq-\frac{\gamma}{4} \psi_{\iota} \geq 0 \quad \text { in } \quad \mathbb{R} .
$$

For $\kappa \leq \kappa^{*}$ let $h_{\kappa}(t, x)$ and $h_{c, \kappa}(t, c)$ be the respectively the solution of

$$
\begin{aligned}
& \partial_{t} h_{\kappa}(t, x)=\mathcal{M}\left[h_{\kappa}\right](t, x)+f\left(x-c t, h_{\kappa}(t, x)\right) \quad \text { for } t>0, \text { and } x \in \mathbb{R}, \\
& h_{\kappa}(0, x)=\kappa \psi_{\iota} \quad \text { on } \quad \mathbb{R} .
\end{aligned}
$$

and

$$
\begin{aligned}
& \partial_{t} h_{c, \kappa}(t, x)=c \mathfrak{D}_{x}\left[h_{c, \kappa}\right](t, x)+\mathcal{M}\left[h_{c, \kappa}\right](t, x)+f\left(x, h_{c, \kappa}(t, x)\right) \quad \text { for } t>0, \text { and } x \in \mathbb{R}, \\
& h_{c, \kappa}(0, x)=\kappa \psi_{\iota}(x) \quad \text { on } \quad \mathbb{R} .
\end{aligned}
$$

By definition since $\psi_{\iota}$ is bounded we can find $\kappa_{0}$ such that for all $\kappa \leq \kappa_{0}, \kappa \psi_{\iota} \leq C\left\|u_{0}\right\|_{\infty}=z_{c}(0, x)=$ $z(0, x)$ and by a straightforward application of the parabolic comparison principle, we see that for all $t>0$ and $x \in \mathbb{R}$

$$
h_{\kappa}(t, x) \leq z(t, x) \quad \text { and } \quad h_{c, \kappa}(t, x) \leq z_{c}(t, x) .
$$

Thanks to Lemma 8.1 the function $h_{c, \kappa}$ is monotone increasing and therefore $z_{c}(t, x)>\kappa \psi_{\iota}$ for all times and $x$. As a consequence the stationary solution $\bar{z}=\bar{u}_{c}>0$ is the unique non trivial stationary solution of (8.3). Similarly, since $h_{c, \kappa}(t, x)$ is increasing and uniformly bounded by $C\left\|u_{0}\right\|_{\infty}$, the positive function $\lim _{t \rightarrow \infty} h_{c, \kappa}(t, x)=\bar{h}(x)$, is well defined for all $x \in \mathbb{R}$ and by standard regularity estimates we can check that $\bar{h}$ the unique positive stationary solution of (8.3) that is $\bar{h}=\bar{u}_{c}$. In addition, we also have $h_{c, \kappa}(t, x) \rightarrow \bar{u}_{c}(x)$ in $C_{l o c}^{1, \alpha}(\mathbb{R})$ as $t \rightarrow+\infty$.

Lastly, let us remark that thanks to the strong maximum principle, we have $u(1, x)>0$ so since $\psi_{\iota}$ is compactly supported and bounded we can find $\kappa_{2}$ such that $u(1, x) \geq \kappa_{2} \psi_{\iota}(x)$ for all $x$. Therefore, by using the uniqueness of the solution of the Cauchy problem and the comparison principle it then standard to obtain that for all $t>0$ and $x \in \mathbb{R} u_{c}(t+1, x) \geq h_{c, \kappa_{2}}(t, x)$. Hence we have

$$
u_{c}(x)=\liminf _{t \rightarrow+\infty} h_{c, \kappa_{2}}(t, x) \leq \liminf u(t, x) \quad \text { for all } \quad x \in \mathbb{R} .
$$

By collecting (8.9) and (8.17) we get for all $x \in \mathbb{R}$

$$
\bar{u}_{c}(x) \leq \liminf _{t \rightarrow \infty} u_{c}(t, x) \leq \limsup _{t \rightarrow \infty} u_{c}(t, x) \leq \limsup _{t \rightarrow \infty} z_{c}(t, x)=\bar{u}_{c}(x) .
$$

8.2. Step Two: Uniform convergence. Now, to complete the proof it remains to show that $\| u_{c}-$ $\bar{u}_{c} \|_{\infty} \rightarrow 0$ as $t \rightarrow \infty$. To this end, we follow an argument used in [13, 4]. We argue by contradiction and assume that there exists $\varepsilon>0$ and sequences $\left(t_{n}\right)_{n \in \mathbb{N}} \in \mathbb{R}^{+},\left(x_{n}\right)_{n \in \mathbb{N}} \in \mathbb{R}$ such that

$$
\lim _{n \rightarrow \infty} t_{n}=\infty, \quad\left|u_{c}\left(t_{n}, x_{n}\right)-\bar{u}_{c}\left(x_{n}\right)\right|>\varepsilon, \quad \forall n \in \mathbb{N} .
$$

By (8.18), we already know that $u_{c} \rightarrow \bar{u}_{c}$ locally uniformly in $\mathbb{R}$, so, without loss of generality, we can assume that $\left|\xi_{n}\right| \rightarrow \infty$. From the construction of $\bar{u}_{c}$, subsection 4.2, we have $\lim _{|x| \rightarrow \infty} \bar{u}_{c}(x)=0$. Therefore, for some $R_{0}>0$, we have $\bar{u}_{x}(x) \leq \frac{\varepsilon}{2}$ for all $x \geq R_{0}$. This, combined with (8.18) and (8.19) enforces

$$
z_{c}\left(t_{n}, x_{n}\right)-\bar{u}_{c}\left(x_{n}\right) \geq u_{c}\left(t_{n}, x_{n}\right)-\bar{u}_{c}\left(x_{n}\right)>\varepsilon, \quad \forall n \in \mathbb{N} .
$$

Next we require the following limiting result

Lemma 8.2. For all sequences $\left(t_{n}\right)_{n \in \mathbb{N}},\left(x_{n}\right)_{n \in \mathbb{N}}$ such that $\lim _{n \rightarrow \infty} t_{n}=\lim _{n \rightarrow \infty}\left|x_{n}\right|=+\infty$, we have $z_{c}\left(t_{n}, x_{n}\right) \rightarrow 0$. 
Assume for the moment that the Lemma holds. Then we obtain a straightforward contradiction since :

$$
0=\lim _{n \rightarrow \infty} z_{c}\left(t_{n}, x_{n}\right)-\bar{u}_{c}\left(x_{n}\right) \geq \lim _{n \rightarrow \infty} u_{c}\left(t_{n}, x_{n}\right)-\bar{u}_{c}\left(x_{n}\right)>\epsilon
$$

We now prove the Lemma.

Proof. Again, we argue by contradiction and assume that there exists $\varepsilon>0$ and sequences $\left(t_{n}\right)_{n \in \mathbb{N}},\left(x_{n}\right)_{n \in \mathbb{N}}$ satisfying $\lim _{n \rightarrow \infty} t_{n}=\lim _{n \rightarrow \infty}\left|x_{n}\right|=\infty$ such that $z\left(t_{n}, x_{n}\right)>\varepsilon$ for all $n \in \mathbb{N}$. Let us define $z_{n}(t, x):=$ $z_{c}\left(t, x+x_{n}\right)$. It satisfies

$$
\begin{aligned}
& \partial_{t} z_{n}(t, x)=c \mathfrak{D}_{x}\left[z_{n}\right](t, x)+\mathcal{M}\left[z_{n}\right](t, x)+f\left(x+x_{n}, z_{n}(t, x)\right) \quad \text { for } t>0, \text { and } x \in \mathbb{R}, \\
& z_{n}(0, x)=C\left\|u_{0}\right\|_{\infty} \quad \text { on } \quad \mathbb{R}^{N},
\end{aligned}
$$

and $0<z_{n}(t, x)<C\left\|u_{0}\right\|_{\infty}$ for $t>0$ and $x \in \mathbb{R}^{N}$. Since for all $n, z_{n}(0, x) \in C^{\infty}$, by the Cauchy Lipschitz Theorem, we see that $z_{n} \in C^{1}\left(\mathbb{R}^{+}, C^{1,1}(\mathbb{R})\right)$. Thus, there exists $C_{0}>0$ independent of $n$ such that $\left\|z_{n}\right\|_{C^{1,1}\left(\mathbb{R}^{+}, C^{1,1}(\mathbb{R})\right)}<C_{0}$. From these estimates, the sequence $\left(z_{n}\right)_{n \in \mathbb{N}}$ is uniformly bounded in $C^{1,1}\left((0, T), C^{1, \alpha}\left(\mathbb{R}^{N}\right)\right)$ for any $T>0$. By a diagonal extraction, there exists a subsequence of $\left(z_{n}\right)_{n \in \mathbb{N}}$ that converges locally uniformly to $\tilde{z}$. Moreover, thanks to $\lim _{|x| \rightarrow \infty} \frac{f(x, s)}{s}<0$, there exists $\kappa>0$ such that $\tilde{z}$ satisfies

$$
\begin{aligned}
& \partial_{t} \tilde{z}(t, x) \leq c \mathfrak{D}_{x}[\tilde{z}](t, x)+\mathcal{M}[\tilde{z}](t, x)-\kappa \tilde{z}(t, x) \quad \text { for } t>0, \text { and } x \in \mathbb{R}, \\
& \tilde{z}(0, x)=C\left\|u_{0}\right\|_{\infty} \quad \text { on } \quad \mathbb{R} .
\end{aligned}
$$

In addition, for all $t>0, \tilde{z}(t, 0)=\lim _{n \rightarrow \infty} z_{n}(t, 0) \geq \varepsilon$. Since $\tilde{z}(0, x)$ is a super-solution of (8.21), by Lemma 8.1 the function $\tilde{z}(t, x)$ is monotone decreasing in time. By sending $t \rightarrow \infty$, since $\tilde{z} \geq 0$, $\tilde{z}$ converges locally uniformly to a non-negative function $\bar{z}$ that satisfies

$$
\begin{aligned}
& c \mathfrak{D}_{x}[\bar{z}]+\mathcal{M}[\bar{z}]-\kappa \bar{z} \geq 0 \quad \text { in } \quad \mathbb{R}, \\
& 0 \leq \bar{z} \leq C\left\|u_{0}\right\|_{\infty}, \\
& \bar{z}(0) \geq \varepsilon .
\end{aligned}
$$

Let us now consider the function $w(x):=\frac{\varepsilon}{2} \cosh (\alpha x)-\bar{z}$ with $\alpha$ to be chosen. A short computation shows that $w$ satisfies

$$
c \mathfrak{D}_{x}[w](x)+\mathcal{M}[w](x)-\kappa w(x) \leq \frac{\varepsilon}{2} \cosh (\alpha x)\left(\operatorname{cotanh}(\alpha x)+\int_{\mathbb{R}} J(z) e^{\alpha z} d y-1-\kappa\right) \quad \text { for } \quad x \in \mathbb{R} .
$$

The left hand side of the inequality is well defined and continuous with respect to $\alpha$ since $J$ is compactly supported. Since $\int_{\mathbb{R}} J(z) d z=1$ and $\alpha|\tanh (\alpha x)| \leq \alpha$, we can find $\alpha$ small such that

$$
c \mathfrak{D}_{x}[w]+\mathcal{M}[w]-\kappa w<0 \quad \text { in } \quad \mathbb{R} .
$$

By construction, since $\bar{z}$ is bounded, $\lim _{|x| \rightarrow \infty} w(x)=+\infty$ and $w$ achieves a minimum in $\mathbb{R}^{N}$, say at $x_{0}$. Since $w(0)=\frac{\varepsilon}{2}-\bar{z}(0) \leq-\frac{\varepsilon}{2}$, we have $w\left(x_{0}\right)<0$. At this point, we get the following contradiction

$$
0<\int_{\mathbb{R}} J\left(x_{0}-y\right)\left[w(y)-w\left(x_{0}\right)\right] d y-\kappa w\left(x_{0}\right)<0 .
$$

\section{FAt TAILED DisPersal KeRnel}

In this last section, we look at the impact of the tail of the kernel $J$ and prove Theorem 1.6. We split this section into two subsection, each one dedicated respectively to the construction of non trivial solution to (1.5) and to the existence of a threshold speed $c^{* *}$ for which no positive solution to (1.5) can exists. In this section, we will always assume that $\sup _{\mathbb{R}} \partial_{s} f(x, 0)<1$ and $J$ is symmetric. 
9.1. Existence of solution. In this subsection, we will show that for any symmetric kernel $J$ there exists $c(J)$ such that for all $|c| \leq c(J)$ then (1.5) has a solution. More precisely,

Lemma 9.1. Assume that $f$ satisfy (1)

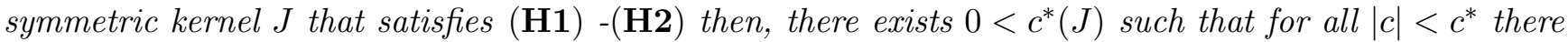
exists a positive solution to (1.5).

Remark 8. Observe that there is no condition on the tail of $J$ for the existence of a positive solution to (1.5).

Proof. Let $\zeta \in C_{c}^{\infty}(\mathbb{R})$ be a cut-off function such that $\zeta(z)=1$ for all $|z| \leq 1, \zeta(z)=0$ for all $|z|>2$, $\zeta(z)=\zeta(-z)$ and $\zeta^{\prime}(z) \leq 0$ for all $z>0$.

For $N \in \mathbb{N}$ define now the function $\zeta_{N}:=\zeta\left(\frac{z}{N}\right)$, the kernel $J_{N}(z):=J(z) \zeta_{N}(z)$ and the operator $\mathcal{M}_{N}$ standing for the operator $\mathcal{M}$ with the kernel $J_{N}$. By definition, we have $J_{N} \leq J$ for all $N$ and $\left(J_{N}\right)_{N \in \mathbb{N}}$ is an increasing sequence of kernel such that $J_{N} \rightarrow J$ pointwise. We now consider the following approximated problem:

$$
c \mathfrak{D}_{x}\left[u_{N}\right]+\mathcal{M}_{N}\left[u_{N}\right]+f\left(x, u_{N}\right)=0 \quad \text { in } \quad \mathbb{R} .
$$

Since $\sup _{x \in \mathbb{R}} \partial_{s} f(x, 0)>1$, thanks to the Proposition 3.2 of [5], we know that for all $N$,

$$
\lambda_{p}\left(\mathcal{M}_{N}+\partial_{\mathbf{s}} \mathbf{f}(\mathbf{x}, \mathbf{0})\right) \leq-\sup _{x \in \mathbb{R}}\left(1-\partial_{s} f(x, 0)\right)<0 .
$$

As a consequence, by Theorem 1.4 since $J_{N}$ is compactly supported and symmetric, there exists $0<c_{N}^{*}$ such that for all $|c| \leq c_{N}^{*}$ the problem (9.1) has a positive solution and

$$
\lambda_{p}\left(c \mathfrak{D}_{x}+\mathcal{M}_{N}+\partial_{\mathbf{s}} \mathbf{f}(\mathbf{x}, \mathbf{0})\right)<0 .
$$

Define $c^{*}:=c_{1}^{*}$, we claim that

Claim 9.2. For all $N \geq 1$ there exists a unique positive solution to (9.1) for all $|c|<c^{*}$.

Assume for the moment that the claim holds true and let us finish our argumentation. Let us fix $c$ such that $|c|<c^{*}$. By the above claim, for all $N$ the equation (9.1) admits a unique positive solution $u_{N}$. Note that since $J_{N}$ is an increasing sequence, $u_{N}$ then satisfies

$$
c \mathfrak{D}_{x}\left[u_{N}\right]+\mathcal{M}_{N+1}\left[u_{N}\right]+f\left(x, u_{N}\right) \geq 0 \quad \text { in } \quad \mathbb{R} .
$$

As a consequence, since $u_{N}$ is bounded, by a standard sweeping argument, we can check that $u_{N} \leq u_{N+1}$,

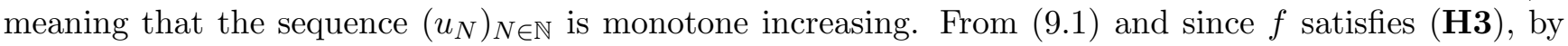
using the maximum principle we can find a universal positive constant $C_{1}$ depending on $f$ such that $\left\|u_{N}\right\|_{\infty}<C_{1}$. By using that $c \neq 0$, we see that $\left(u_{N}\right)_{N \in \mathbb{N}}$ is bounded uniformly in $C_{l o c}^{1, \alpha}(\mathbb{R})$ and therefore by a diagonal extraction process we can extract of the non decreasing sequence $\left(u_{N}\right)_{N \in \mathbb{N}}$ an non decreasing subsequence, still denoted $\left(u_{N}\right)_{N \in \mathbb{N}}$ that converges locally uniformly to a positive bounded function $u$ which is solution to (1.5). The above argument being independent of $c$, we then obtain a positive solution to (1.5) for all $|c|<c^{*}$ and the Lemma is proved.

To complete the argument of the Lemma, let us now prove the claim.

Proof of the claim. We make an inductive argument. For $N=1$ then since $c^{*}=c_{1}^{*}$ for all $|c|<c^{*}$ then the problem (9.1) has a unique solution with $\mathcal{M}_{1}$. Let us assume that for some $N \geq 1$ then the problem (9.1) with the operator $\mathcal{M}_{N}$ has a unique solution for all $|c|<c^{*}$ and let us prove that this is still true for $N+1$.

Let $-c^{*}<c<c^{*}$ be fixed, then by assumption there exists $u_{N}>0$ a solution to (9.1) with the operator $\mathcal{M}_{N}$ and thanks to Theorem 1.1 and Theorem 1.2 we must have

$$
\lambda_{p}\left(c+\mathcal{M}_{N}+\partial_{\mathbf{s}} \mathbf{f}(\mathbf{x}, \mathbf{0})\right)<0 .
$$


Since $J_{N}$ is an increasing sequence, thanks to the monotone behaviour of the principal eigenvalue, it follows that

$$
\lambda_{p}\left(c \mathfrak{D}_{x}+\mathcal{M}_{N+1}+\partial_{\mathbf{s}} \mathbf{f}(\mathbf{x}, \mathbf{0})\right) \leq \lambda_{p}\left(c \mathfrak{D}_{x}+\mathcal{M}_{N}+\partial_{\mathbf{s}} \mathbf{f}(\mathbf{x}, \mathbf{0})\right)<0 .
$$

We now treat the three cases $c>, c=0$ and $c<0$ separately.

Case $c>0:$. In this situation, by Theorem 1.1 we readily conclude that there exists a unique solution to the problem (9.1) with $\mathcal{M}_{N+1}$, that is a positive solution to

$$
c \mathfrak{D}_{x}[u]+\mathcal{M}_{N+1}[u]+f(x, u)=0 \quad \text { in } \quad \mathbb{R} .
$$

Case $c=0$ : In this situation the existence of a positive solution is already known thanks to [5].

Case $c<0$ : In this situation, let us observe that by using Theorem 1.2, we have

$$
0>\lambda_{p}\left(c \mathfrak{D}_{x}+\mathcal{M}_{N+1}+\partial_{\mathbf{s}} \mathbf{f}(\mathbf{x}, \mathbf{0})\right)=\lambda_{p}\left(-c \mathfrak{D}_{x}+\mathcal{M}_{N+1}^{*}+\partial_{\mathbf{s}} \mathbf{f}(-\mathbf{x}, \mathbf{0})\right) .
$$

Therefore by using Theorem 1.1, we can check that there exists a unique solution to

$$
-c \mathfrak{D}_{x}[v]+\mathcal{M}_{N+1}^{*}[v]+f(-x, v)=0 \quad \text { in } \quad \mathbb{R} .
$$

Now by taking $u(x):=v(-x)$, a short computation shows that $u$ is then a solution to (9.2).

In summary, in all situations, there exists a positive solution to (9.1) with the operator $\mathcal{M}_{N+1}$. Since the above argument is independent of $c$, we conclude that there exists a solution to (9.1) with the operator $\mathcal{M}_{N+1}$ for all $|c|<c^{*}$. The claim then follows by induction.

9.2. An upper bound of the speed. As for compactly supported kernel, we will obtain in this subsection an estimate on the critical speed $c^{* *}$ for fat-tailed kernel that satisfies the additional assumption

$$
\int_{\mathbb{R}} J(z)|z|^{2} d z<+\infty
$$

Namely, let us consider the $C^{1}$ function

$$
w_{\tau}(x):= \begin{cases}1-\tau x & \text { when } x \leq 0, \\ \frac{1}{1+\tau x} & \text { when } x \geq 0 .\end{cases}
$$

To obtain our bound, it then enough to verify that for $c>>1$ we can find $\tau>0$ and $\delta>0$ such that $w_{\tau}$ satisfies:

$$
c \mathfrak{D}_{x}\left[w_{\tau}\right](x)+\mathcal{M}\left[w_{\tau}\right](x)+(a(x)+\delta) w_{\tau}(x) \leq 0 \quad \text { for all } \quad x \in \mathbb{R} .
$$

Indeed, by definition of the principal eigenvalue the above inequality then implies that $\lambda_{p}\left(c \mathfrak{D}_{x}+\mathcal{M}+\right.$ $\mathbf{a}(\mathbf{x}))>0$, which in turn implies the non existence of a positive solution as proved in Section 7 .

So let us compute $\mathcal{R}\left[w_{\tau}\right]:=c \mathfrak{D}_{x}\left[w_{\tau}\right](x)+\mathcal{M}\left[w_{\tau}\right](x)+a(x) w_{\tau}(x)$. For $x \leq 0$, we then have

$$
\begin{aligned}
\mathcal{R}\left[w_{\tau}\right](x) & =-c \tau-\tau \int_{-\infty}^{-x} J(z) z d z+\int_{0}^{+\infty} J(x-y)\left[\frac{1}{1+\tau y}-1+\tau x\right] d y+a(x)(1-\tau x) \\
& =-c \tau+I_{1}+I_{2}+a(x)(1-\tau x) .
\end{aligned}
$$

Observe that thanks to the symmetry of $J$ we can estimate $I_{1}$ by

$$
I_{1}=-\tau \int_{-\infty}^{x} J(z) z d z=\tau \int_{-x}^{+\infty} J(z) z d z
$$

Let us now estimate $I_{2}$, by a direct computation since $x \leq 0$ we have

$$
\begin{aligned}
I_{2} & =\int_{0}^{+\infty} J(x-y) \frac{1-(1-\tau x)(1+\tau y)}{1+\tau y} d y \\
& =\tau \int_{0}^{+\infty} J(x-y) \frac{x-y}{1+\tau y} d y+\tau x \int_{0}^{+\infty} J(x-y) \frac{\tau y}{1+\tau y} d y \\
& =-\tau \int_{-x}^{+\infty} J(z) \frac{z}{1+\tau(x+z)} d y+\tau x \int_{0}^{+\infty} J(x-y) \frac{\tau y}{1+\tau y} d y \\
& \leq 0 .
\end{aligned}
$$


Therefore we have

$$
\mathcal{R}\left[w_{\tau}\right](x) \leq \tau\left(-c+\int_{-x}^{+\infty} J(z) z d z\right)+a(x) w_{\tau}(x) .
$$

On the other hand, for $x \geq 0$ we have

$$
\begin{aligned}
\mathcal{R}\left[w_{\tau}\right](x) & =\left(-\frac{c \tau}{1+\tau x}+\int_{-\infty}^{0} J(x-y)\left[\tau(x-y)-\tau^{2} x y\right] d y+\int_{0}^{+\infty} J(x-y)\left[\frac{1+\tau x}{1+\tau y}-1\right] d y+a(x)\right) w_{\tau}(x) \\
& =\left(-\frac{c \tau}{1+\tau x}+I_{3}+I_{4}+a(x)\right) w_{\tau}(x) .
\end{aligned}
$$

Let us estimate $I_{3}$ and $I_{4}$. First observe that by a direct computation we have

$$
I_{4}=\int_{0}^{+\infty} J(x-y) \frac{\tau(x-y)}{1+\tau y}=-\tau \int_{-x}^{+\infty} J(z) \frac{z}{1+\tau(x+z)} d z \leq \tau \int_{-x}^{0} J(z) z d z=\tau \int_{0}^{x} J(z) z d z .
$$

Let us estimate $I_{3}$, again a direct computation gives

$$
\begin{aligned}
I_{3}=\int_{-\infty}^{-x} J(z)\left[-\tau z-\tau^{2} x(x+z)\right] d z & =\tau \int_{x}^{+\infty} J(z) z d z-\int_{x}^{+\infty} J(z)\left[\tau^{2} x(x-z)\right] d z \\
& \leq \tau \int_{x}^{+\infty} J(z) z d z+\tau^{2} x \int_{x}^{+\infty} J(z) z d z \\
& \leq \tau \int_{x}^{+\infty} J(z) z d z+\tau^{2} \int_{x}^{+\infty} J(z) z^{2} d z .
\end{aligned}
$$

Therefore we have

$$
\mathcal{R}\left[w_{\tau}\right](x) \leq\left(-\frac{c \tau}{1+\tau x}+\tau M_{1}+\tau^{2} M_{2}+a(x)\right) w_{\tau}(x)
$$

where the constant $M_{1}$ and $M_{2}$ refers to

$$
M_{1}:=\int_{0}^{+\infty} J(z) z d z, \quad M_{2}:=\int_{0}^{+\infty} J(z) z^{2} d z .
$$

Now since $f$ satisfies $(\underline{\mathbf{H 3}})$ - $(\mathbf{H 5})$ then there exists $R_{0}>0, \delta>0, \kappa>0$ such that $a(x)+\delta \leq-\kappa$ for all $|x| \geq R_{0}$. Let $\tau_{0}:=\frac{-M_{1}+\sqrt{M_{1}^{2}+4 \kappa M_{2}}}{2 M_{2}}$, then $\tau_{0}$ satisfies $\tau^{2} M_{2}+\tau M_{1}-\kappa=0$ and for any $c \geq 0$ we can check from (9.4) that for $x \geq R_{0}$

$$
\mathcal{R}\left[w_{\tau_{0}}\right](x)+\delta w_{\tau_{0}}(x) \leq-\frac{c \tau}{1+\tau x} w_{\tau_{0}}(x) \leq 0
$$

Similarly thanks to (9.3) for $c \geq c_{0}:=\int_{0}^{+\infty} J(z) z d z$ we have for all $\tau \geq 0$ and all $x \leq-R_{0}$

$$
\mathcal{R}\left[w_{\tau_{0}}\right](x)+\delta w_{\tau_{0}}(x) \leq-\tau \int_{0}^{-x} J(z) z d z+(a(x)+\delta) w_{\tau_{0}}(x) \leq 0,
$$

To conclude, it remains to find $c$ large such that the inequality holds for $|x| \leq R_{0}$ and $\tau=\tau_{0}$. By taking $c \geq c_{1}:=c_{0}+\sup _{x \in \mathbb{R}}(a(x)+\delta) \frac{1+\tau_{0} R_{0}}{\tau_{0}}$ and by using (9.3) we can check that for $-R_{0} \leq x \leq 0$ we have

$$
\mathcal{R}\left[w_{\tau_{0}}\right](x)+\delta w_{\tau_{0}}(x) \leq-\tau_{0} \int_{0}^{-x} J(z) z d z+(a(x)+\delta) w_{\tau_{0}}(x)-\sup _{x \in \mathbb{R}}(a(x)+\delta) \sup _{x \in\left[0, R_{0}\right]} w_{\tau_{0}}(x) \leq 0
$$


Whereas for $c \geq c_{2}:=\left(\kappa+\delta+\sup _{x \in \mathbb{R}} a(x)\right) \frac{1+\tau_{0} R_{0}}{\tau_{0}}$ and for $0 \leq x \leq R_{0}$, thanks to (9.4), we have

$$
\mathcal{R}\left[w_{\tau_{0}}\right](x)+\delta w_{\tau_{0}}(x) \leq\left(-\frac{c \tau_{0}}{1+\tau_{0} R_{0}}+\kappa+\delta+\sup _{x \in \mathbb{R}} a(x)\right) w_{\tau_{0}}(x) \leq 0 .
$$

By collecting (9.5), (9.6), (9.7) and (9.8), for $c \geq c^{\#}:=\sup \left\{c_{0}, c_{1}, c_{2}\right\}$ we can see that the function $w_{\tau_{0}}$ satisfies

$$
\mathcal{R}\left[w_{\tau_{0}}\right](x)+\delta w_{\tau_{0}}(x) \leq 0 \quad \text { for all } \quad x \in \mathbb{R}
$$

\section{ACKNowledgements}

The author warmly thanks professors Salome Martínez and Juan Dávila for their exceptional hospitality at the Departamento de Ingeniería Matemática of the Universidad de Chile where this research have started during my last visit to the Centro de Modelamiento Matemático (CMM). The author would also thanks Florian Patout (INRAE BioSP) for enlighting discussion on this subject. The author acknowledges also support from the "ANR DEFI" project NONLOCAL: ANR-13-JS01-0009.

\section{REFERENCES}

1. Lee Altenberg, Resolvent positive linear operators exhibit the reduction phenomenon, Proceedings of the National Academy of Sciences 109 (2012), no. 10, 3705-3710.

2. M. Bakkenes, J.R.M. Alkemade, F. Ihle, R. Leemans, and J.B. Latour, Assessing effects of forecasted climate change on the diversity and distribution of european higher plants for 2050, Global change biology 8 (2002), no. 4, 390-407.

3. P. W. Bates and G. Zhao, Existence, uniqueness and stability of the stationary solution to a nonlocal evolution equation arising in population dispersal, J. Math. Anal. Appl. 332 (2007), no. 1, 428-440. MR MR2319673 (2008h:35019)

4. H. Berestycki, J. Coville, and H-H. Vo, On the definition and the properties of the principal eigenvalue of some nonlocal operators, Journal of Functional Analysis 271 (2016), no. 10, 2701 - 2751.

5. H. Berestycki, J. Coville, and H-H. Vo, Persistence criteria for populations with non-local dispersion, Journal of Mathematical Biology 72 (2016), no. 7, 1693-1745.

6. H. Berestycki, O. Diekmann, C. J. Nagelkerke, and P. A. Zegeling, Can a species keep pace with a shifting climate?, Bull. Math. Biol. 71 (2009), no. 2, 399-429. MR 2471053 (2010a:92052)

7. H. Berestycki, F. Hamel, and L. Roques, Analysis of the periodically fragmented environment model. I. Species persistence, J. Math. Biol. 51 (2005), no. 1, 75-113. MR MR2214420

8. H. Berestycki, F. Hamel, and L. Rossi, Liouville-type results for semilinear elliptic equations in unbounded domains, Ann. Mat. Pura Appl. (4) 186 (2007), no. 3, 469-507. MR MR2317650 (2008c:35058)

9. H. Berestycki, B. Larrouturou, and P.-L. Lions, Multi-dimensional travelling-wave solutions of a flame propagation model, Arch. Rational Mech. Anal. 111 (1990), no. 1, 33-49. MR MR1051478 (91h:35148)

10. H. Berestycki and L. Nirenberg, Travelling fronts in cylinders, Ann. Inst. H. Poincaré Anal. Non Linéaire 9 (1992), no. 5, 497-572. MR MR1191008 (93k:35019)

11. H. Berestycki, L. Nirenberg, and S. R. S. Varadhan, The principal eigenvalue and maximum principle for second-order elliptic operators in general domains, Comm. Pure Appl. Math. 47 (1994), no. 1, 47-92. MR MR1258192 (95h:35053)

12. H. Berestycki and L. Rossi, On the principal eigenvalue of elliptic operators in $\mathbb{R}^{N}$ and applications, J. Eur. Math. Soc. (JEMS) 8 (2006), no. 2, 195-215. MR MR2239272 (2007d:35076)

13. _ Reaction-diffusion equations for population dynamics with forced speed. I. The case of the whole space, Discrete Contin. Dyn. Syst. 21 (2008), no. 1, 41-67. MR 2379456 (2009f:35173)

14. L_ Reaction-diffusion equations for population dynamics with forced speed ii - cylindrical-type domains, Discrete and Continuous Dynamical Systems 25 (2009), no. 1, 19-61.

15. __ Generalizations and properties of the principal eigenvalue of elliptic operators in unbounded domains, Communications on Pure and Applied Mathematics 68 (2015), no. 6, 1014-1065.

16. E. Bouin, T. Bourgeron, V. Calvez, O. Cotto, J. Garnier, T. Lepoutre, and O. Ronce, Equilibria of quantitative genetics models beyond the gaussian approximation $i$ : Maladaptation to a changing environment, 2018.

17. E. O. Box, Predicting physiognomic vegetation types with climate variables, Vegetatio 45 (1981), no. 2, $127-139$.

18. J. Brasseur, The role of the range of dispersal in a nonlocal fisher-kpp equation: an asymptotic analysis, Communications in Contemporary Mathematics (2020), 2050032.

19. M. L. Cain, B. G. Milligan, and A. E. Strand, Long-distance seed dispersal in plant populations, Am. J. Bot. 87 (2000), no. 9, 1217-1227.

20. J. S. Clark, Why trees migrate so fast: Confronting theory with dispersal biology and the paleorecord, The American Naturalist 152 (1998), no. 2, 204-224. 
21. J. S. Clark, C. Fastie, G. Hurtt, S.T. Jackson, C. Johnson, G.A. King, M. Lewis, J. Lynch, S. Pacala, C. Prentice, E. Schupp, T. Webb III, and P. Wyckoff, Reid's paradox of rapid plant migration, BioScience 48 (1998), 13-24.

22. B. Cloez and P. Gabriel, On an irreducibility type condition for the ergodicity of nonconservative semigroups, Comptes Rendus. Mathématique 358 (2020), no. 6, 733-742 (en).

23. J. Coville, Travelling fronts in asymmetric nonlocal reaction diffusion equations: The bistable and ignition cases, CCSDHal e-print (2007), -.

24. _ On a simple criterion for the existence of a principal eigenfunction of some nonlocal operators, J. Differential Equations 249 (2010), no. 11, 2921 - 2953.

25. Nonlocal refuge model with a partial control, Discrete and Continuous Dynamical Systems 35 (2015), no. 4, 1421-1446.

26. J. Coville, J. Davila, and S. Martinez, Existence and uniqueness of solutions to a nonlocal equation with monostable nonlinearity, SIAM Journal on Mathematical Analysis 39 (2008), no. 5, 1693-1709.

27. Nonlocal anisotropic dispersal with monostable nonlinearity, J. Differential Equations 244 (2008), no. 12, 30803118. MR MR2420515

28. _ Pulsating fronts for nonlocal dispersion and KPP nonlinearity, Ann. I. H. Poincare - AN (2013), no. 30, $179-223$.

29. J. Coville and L. Dupaigne, On a non-local equation arising in population dynamics, Proc. Roy. Soc. Edinburgh Sect. A 137 (2007), no. 4, 727-755. MR MR2345778

30. J. Coville and F. Hamel, On generalized principal eigenvalues of nonlocal operators witha drift, Nonlinear Analysis 193 (2020), 111569, Nonlocal and Fractional Phenomena.

31. J. Coville, F. Li, and X. Wang, On eigenvalue problems arising from nonlocal diffusion models, Discrete and Continuous Dynamical Systems, series A 37 (2017), no. 2, 879-903.

32. P. De Leenheer, W. Shen, and A. Zhang, Persistence and extinction of nonlocal dispersal evolution equations in moving habitats, Nonlinear Analysis: Real World Applications 54 (2020), 103110.

33. P. C. Fife, An integrodifferential analog of semilinear parabolic PDEs, Partial differential equations and applications, Lecture Notes in Pure and Appl. Math., vol. 177, Dekker, New York, 1996, pp. 137-145. MR MR1371585 (97c:35092)

34. J. Garcia-Melian and J. D. Rossi, A logistic equation with refuge and nonlocal diffusion, Commun. Pure Appl. Anal. 8 (2009), no. 6, 2037-2053. MR 2552163 (2010k:45002)

35. M. Grinfeld, G. Hines, V. Hutson, K. Mischaikow, and G. T. Vickers, Non-local dispersal, Differential Integral Equations 18 (2005), no. 11, 1299-1320. MR MR2174822 (2006m:35033)

36. A. Guisan and W. Thuiller, Predicting species distribution: offering more than simple habitat models, Ecology letters $\mathbf{8}$ (2005), no. 9, 993-1009.

37. S. Hapca, J. W. Crawford, and I. M. Young, Anomalous diffusion of heterogeneous populations characterized by normal diffusion at the individual level, Journal of The Royal Society Interface 6 (2009), no. 30, 111-122.

38. M. A Harsch, Y. Zhou, J. HilleRisLambers, and M. Kot, Keeping pace with climate change: stage-structured movinghabitat models, The American Naturalist 184 (2014), no. 1, 25-37.

39. L. Hughes, Biological consequences of global warming: is the signal already apparent?, Trends in ecology \& evolution 15 (2000), no. 2, 56-61.

40. V. Hutson, S. Martinez, K. Mischaikow, and G. T. Vickers, The evolution of dispersal, J. Math. Biol. 47 (2003), no. 6 , 483-517. MR MR2028048 (2004j:92074)

41. C-Y. Kao, Y. Lou, and W. Shen, Random dispersal vs. nonlocal dispersal, Discrete and Continuous Dynamical Systems 26 (2010), no. 2, 551-596.

42. W-T. Li, J-B. Wang, and X-Q. Zhao, Spatial dynamics of a nonlocal dispersal population model in a shifting environment, Journal of Nonlinear Science 28 (2018), no. 4, 1189-1219.

43. F. Lutscher, E. Pachepsky, and M. Lewis, The effect of dispersal patterns on stream populations, SIAM Rev. 47 (2005), no. 4, 749-772 (electronic). MR MR2212398 (2006k:92082)

44. J. P. McCarty, Ecological consequences of recent climate change, Conservation biology 15 (2001), no. 2, $320-331$.

45. R. D. Nussbaum and Y. Pinchover, On variational principles for the generalized principal eigenvalue of second order elliptic operators and some applications, J. Anal. Math. 59 (1992), 161-177, Festschrift on the occasion of the 70th birthday of Shmuel Agmon. MR MR1226957 (94h:35049)

46. C. Parmesan, N. Ryrholm, C. Stefanescu, J. K. Hill, C. D. Thomas, H. Descimon, B. Huntley, L. Kaila, J. Kullberg, T. Tammaru, et al., Poleward shifts in geographical ranges of butterfly species associated with regional warming, Nature 399 (1999), no. 6736, 579-583.

47. S. Petrovskii, A. Mashanova, and V. A.A. Jansen, Variation in individual walking behavior creates the impression of a lévy flight, Proceedings of the National Academy of Sciences 108 (2011), no. 21, 8704-8707.

48. A. B. Potapov and M. A. Lewis, Climate and competition: the effect of moving range boundaries on habitat invasibility, Bull. Math. Bio. 66 (2004), no. 5, 975-1008.

49. N. Rawal and W. Shen, Criteria for the existence and lower bounds of principal eigenvalues of time periodic nonlocal dispersal operators and applications, Journal of Dynamics and Differential Equations 24 (2012), no. 4, 927-954 (English).

50. L. Roques, F. Patout, O. Bonnefon, and G. Martin, Adaptation in general temporally changing environments, arXiv preprint arXiv:2002.09542 (2020). 
51. F. M. Schurr, O. Steinitz, and R. Nathan, Plant fecundity and seed dispersal in spatially heterogeneous environments: models, mechanisms and estimation, J. Ecol. 96 (2008), no. 4, 628-641.

52. W. Shen and X. Xie, On principal spectrum points/principal eigenvalues of nonlocal dispersal operators and applications, Discrete and Continuous Dynamical Systems 35 (2015), no. 4, 1665-1696.

53. W. Shen and A. Zhang, Stationary solutions and spreading speeds of nonlocal monostable equations in space periodic habitats., Proc. Am. Math. Soc. 140 (2012), no. 5, 1681-1696 (English).

54. P. Turchin, Quantitative analysis of movement: Measuring and modeling population redistribution in animals and plants, Sinauer Associates, 1998.

55. A. Velleret, Adaptation of a population to a changing environment under the light of quasi-stationarity, arXiv: Probability (2019).

56. H-H. Vo, Persistence versus extinction under a climate change in mixed environments, Journal of Differential Equations 259 (2015), no. 10, 4947 - 4988.

57. G-R. Walther, E. Post, P. Convey, A. Menzel, C. Parmesan, T.J.C. Beebee, J-M. Fromentin, O. Hoegh-Guldberg, and F. Bairlein, Ecological responses to recent climate change, Nature 416 (2002), no. 6879, 389-395.

58. Y. Zhou and M. Kot, Discrete-time growth-dispersal models with shifting species ranges, Theoretical Ecology 4 (2011), no. 1, 13-25.

59. L Life on the move: modeling the effects of climate-driven range shifts with integrodifference equations, Dispersal, individual movement and spatial ecology, Springer, 2013, pp. 263-292.

BioSP, INRAE, 84914, Avignon, France

Email address: jerome.coville@inrae.fr 